Supporting Information

\title{
Annulation Cascade of Sulfamate-Derived Cyclic Imines with Glycine Aldimino Esters: Synthesis of 1,3-Benzoxazepine Scaffolds
}

Mallu Kesava Reddy, Vinod Bhajammanavar, and Mahiuddin Baidya*

\author{
Department of Chemistry, Indian Institute of Technology Madras, \\ Chennai 600 036, Tamil Nadu, India \\ E-mail:mbaidya@iitm.ac.in
}


Table of Contents

Page

General procedure

General procedure for the synthesis of 1,3-Benzoxazepines 4-5

Procedure for the gram scale synthesis of compound $\mathbf{5 s}$

Procedure for the synthesis of ortho-tyrosine amino acid derivatives 6 


\section{General Procedure}

All non-aqueous reactions were carried out under an atmosphere of nitrogen in flame-dried glassware and were stirred using a magnetic stir plate. All reactions were carried out using commercial grade solvent unless otherwise noted. $\mathrm{CH}_{3} \mathrm{CN}$, DCE, and DCM, were dried over calcium hydride. Dry THF was prepared by distilling over sodium ketyl.

All reactions were monitored by thin layer chromatography (TLC) on WhatmanPartisil@ K6F TLC plates (silica gel $60 \AA, 0.25 \mathrm{~mm}$ thickness) and visualized using a UV lamp (366 or $254 \mathrm{~nm}$ ) or by use of one of the following visualization reagents: PMA: $10 \mathrm{~g}$ phosphomolybdic acid/ $100 \mathrm{~mL}$ ethanol; $\mathrm{KMnO}_{4}: 0.75 \mathrm{~g}$ potassium permanganate, $5 \mathrm{~g} \mathrm{~K}_{2} \mathrm{CO}_{3}$, / 100mL water. Products were isolated by column chromatography (Merck silica gel 100$200 \mu \mathrm{m}$ ). Yields refer to chromatographically and spectroscopically homogenous materials unless noted otherwise. ${ }^{13} \mathrm{C}$ and ${ }^{1} \mathrm{H}$ NMR spectra were recorded on a Bruker 400 or Bruker $500 \mathrm{MHz}$ spectrometers. Chemical shift values ( $\delta$ ) are reported in ppm and calibrated to the residual solvent peak $\mathrm{CDCl}_{3} \delta=7.2600 \mathrm{ppm}$ for ${ }^{1} \mathrm{H}, \delta=77.16$ for ${ }^{13} \mathrm{C}$ or calibrated to tetramethylsilane $(\delta=0.00)$. All NMR spectra were recorded at ambient temperature $(290 \mathrm{~K})$ unless otherwise noted. ${ }^{1} \mathrm{H}$ NMR spectra are reported as follows: chemical shift (multiplicity, coupling constant, integration). The following abbreviations are used to indicate multiplicities: $s$, singlet; $d$, doublet; $t$, triplet; $q$, quartet; quint, quintet; sext, sextet; sept, septet; m, multiplet; dd, doublet of doublet; dt, doublet of triplet; dq, doublet of quartet; td, triplet of doublet; tt, triplet of triplet; dq, doublet of quartet; ddt, doudlet of doublet of triplet; b, broad; app, apparent. Mass spectra were recorded by electrospray ionization (ESI) method on a Q-TOF Micro with lock spray source. The crystal data were collected and integrated using a BrukerAxs kappa apex2 CCD diffractometer, with graphite monochromated Mo-Ka radiation.

The sulfamate derived cyclic imines 1 were synthesized by following literature procedures (Asian J. Org. Chem. 2018, 7, 2452; J. Org. Chem. 2016, 81, 8561; Angew Chem. Int. Ed. 2009, 48, 4513). Glycine aldimino esters 2 were prepared by following literature procedures (Green Chem. 2019, 21, 4609; J. Am. Chem. Soc. 2009, 141, 961; J. Org. Chem. 2021, 86, 4361).

General procedure for the synthesis of 1,3-Benzoxazepines 4-5:
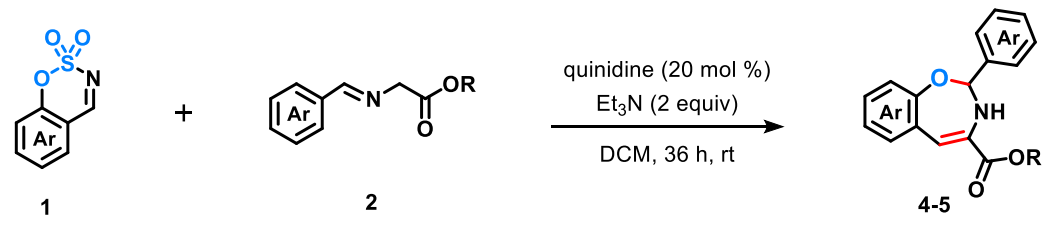

In an oven dried reaction tube equipped with a magnetic stir bar, sulfamate-derived imine $\mathbf{1}(0.2 \mathrm{mmol}, 1.0$ equiv), glycine aldimino ester 2 ( $0.22 \mathrm{mmol}, 1.1$ equiv), and quinidine (20 mol \%) were taken. Then, dry DCM ( $2 \mathrm{~mL})$, followed dry $\mathrm{Et}_{3} \mathrm{~N}$ (2.0 equiv) were added and the reaction mixture was stir at room temperature. After $36 \mathrm{~h}$ (progress of the reaction was monitored by thin layer chromatography) reaction mixture was concentrated under reduced pressure and the crude product was purified by silica gel column chromatography $(1 \rightarrow 5 \%$ EtOAc : hexane) to give pure product 4-5.

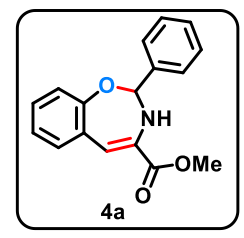

Compound 4a was prepared following the general procedure for synthesis of 1,3Benzoxazepines described above by using $1 \mathbf{a}(0.22 \mathrm{mmol}, 36 \mathrm{mg})$ and purified by silica gel column chromatography $(1 \rightarrow 5 \%$ EtOAc : hexane) to provide pure compound as yellow solid $(48 \mathrm{mg}, 86 \%)$, melting point $=86-88{ }^{\circ} \mathrm{C} .{ }^{1} \mathbf{H}$ NMR $\left(400 \mathrm{MHz}, \mathrm{CDCl}_{3}\right) \delta$ $7.58-7.56(\mathrm{~m}, 2 \mathrm{H}), 7.48-7.43(\mathrm{~m}, 3 \mathrm{H}), 7.29(\mathrm{~d}, J=7.4 \mathrm{~Hz}, 1 \mathrm{H}), 7.14-7.11(\mathrm{~m}, 1 \mathrm{H})$, $7.05-6.98(\mathrm{~m}, 2 \mathrm{H}), 6.63(\mathrm{~s}, 1 \mathrm{H}), 5.63(\mathrm{~s}, 1 \mathrm{H}), 5.54(\mathrm{~d}, J=4.6 \mathrm{~Hz}, 1 \mathrm{H}), 3.86(\mathrm{~s}, 3 \mathrm{H}) .{ }^{13} \mathrm{C}$ NMR $\left(101 \mathrm{MHz}, \mathrm{CDCl}_{3}\right) \delta 165.2,159.1,138.5,132.8,132.6,129.2,129.0,127.8,126.7$, 125.9, 123.2, 120.7, 110.6, 88.5, 52.8. HRMS (ESI/TOF-Q) $\mathrm{m} / \mathrm{z}:[\mathrm{M}+\mathrm{H}]^{+}$Calcd for $\mathrm{C}_{17} \mathrm{H}_{15} \mathrm{NO}_{3} \mathrm{H}^{+} 282.1125$; Found 282.1129. 

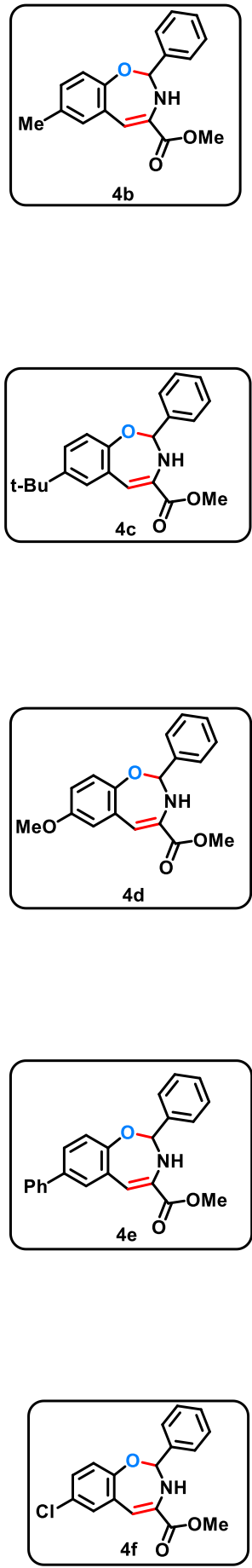

Compound 4b was prepared following the general procedure for synthesis of 1,3Benzoxazepines described above by using $\mathbf{1 b}(0.22 \mathrm{mmol}, 39 \mathrm{mg})$ and purified by silica gel column chromatography $(1 \rightarrow 5 \%$ EtOAc : hexane) to provide pure compound as yellow solid $(53 \mathrm{mg}, 89 \%)$, melting point $=102-104{ }^{\circ} \mathrm{C} .{ }^{1} \mathbf{H}$ NMR $\left(400 \mathrm{MHz}, \mathrm{CDCl}_{3}\right) \delta$ $7.57-7.55(\mathrm{~m}, 2 \mathrm{H}), 7.45-7.44(\mathrm{~m}, 3 \mathrm{H}), 7.09(\mathrm{~s}, 1 \mathrm{H}), 6.94-6.87(\mathrm{~m}, 2 \mathrm{H}), 6.59(\mathrm{~s}, 1 \mathrm{H})$, $5.60(\mathrm{~s}, 1 \mathrm{H}), 5.51(\mathrm{~d}, J=4.3 \mathrm{~Hz}, 1 \mathrm{H}), 3.85(\mathrm{~s}, 3 \mathrm{H}), 2.30(\mathrm{~s}, 3 \mathrm{H}) .{ }^{13} \mathbf{C}$ NMR $(101 \mathrm{MHz}$, $\left.\mathrm{CDCl}_{3}\right) \delta 165.2,157.0,138.6,132.8,132.7,132.4,129.2,129.0,128.6,126.7,125.6$, 120.5, 110.7, 88.5, 52.7, 20.6. HRMS (ESI/TOF-Q) m/z: $[\mathrm{M}+\mathrm{Na}]^{+}$Calcd for $\mathrm{C}_{18} \mathrm{H}_{17} \mathrm{NO}_{3} \mathrm{Na}^{+} 318.1101$; Found 318.1110.

Compound $\mathbf{4 c}$ was prepared following the general procedure for synthesis of 1,3Benzoxazepines described above by using $1 \mathrm{c}(0.22 \mathrm{mmol}, 48 \mathrm{mg})$ and purified by silica gel column chromatography $(1 \rightarrow 5 \%$ EtOAc : hexane) to provide pure compound as yellow solid $(58 \mathrm{mg}, 86 \%)$, melting point $=94-96{ }^{\circ} \mathrm{C} .{ }^{1} \mathbf{H}$ NMR $\left(400 \mathrm{MHz}, \mathrm{CDCl}_{3}\right) \delta$ $7.47(\mathrm{~d}, J=7.1 \mathrm{~Hz}, 2 \mathrm{H}), 7.38-7.33(\mathrm{~m}, 3 \mathrm{H}), 7.20(\mathrm{bs}, 1 \mathrm{H}), 7.07(\mathrm{~d}, J=8.4 \mathrm{~Hz}, 1 \mathrm{H})$, $6.84(\mathrm{~d}, J=8.4 \mathrm{~Hz}, 1 \mathrm{H}), 6.56(\mathrm{~s}, 1 \mathrm{H}), 5.50(\mathrm{~s}, 1 \mathrm{H}), 5.44(\mathrm{~d}, J=4.2 \mathrm{~Hz}, 1 \mathrm{H}), 3.76(\mathrm{~s}, 3 \mathrm{H})$, 1.23 (s, 9H). ${ }^{13} \mathrm{C}$ NMR $\left(101 \mathrm{MHz}, \mathrm{CDCl}_{3}\right) \delta 165.3,156.9,145.8,138.6,132.6,129.3$, 129.2, 129.0, 126.7, 125.1, 125.0, 120.2, 111.3, 88.4, 52.7, 34.2, 31.5. HRMS (ESI/TOFQ) $\mathrm{m} / \mathrm{z}:[\mathrm{M}+\mathrm{Na}]^{+}$Calcd for $\mathrm{C}_{21} \mathrm{H}_{23} \mathrm{NO}_{3} \mathrm{Na}^{+} 360.1570$; Found 360.1581 .

Compound 4d was prepared following the general procedure for synthesis of 1,3Benzoxazepines described above by using $1 \mathbf{1 d}(0.22 \mathrm{mmol}, 43 \mathrm{mg})$ and purified by silica gel column chromatography ( $\rightarrow 5 \%$ EtOAc : hexane) to provide pure compound as yellow solid $(53 \mathrm{mg}, 85 \%)$, melting point $=102-104{ }^{\circ} \mathrm{C} .{ }^{1} \mathbf{H}$ NMR $\left(400 \mathrm{MHz}, \mathrm{CDCl}_{3}\right) \delta$ $7.56-7.55(\mathrm{~m}, 2 \mathrm{H}), 7.47-7.42(\mathrm{~m}, 3 \mathrm{H}), 6.91(\mathrm{~d}, J=8.8 \mathrm{~Hz}, 1 \mathrm{H}), 6.92-6.90(\mathrm{~m}, 1 \mathrm{H})$, $6.70-6.67(\mathrm{~m}, 1 \mathrm{H}), 6.56(\mathrm{~s}, 1 \mathrm{H}), 5.63(\mathrm{~s}, 1 \mathrm{H}), 5.49(\mathrm{~d}, J=4.6 \mathrm{~Hz}, 1 \mathrm{H}), 3.85(\mathrm{~s}, 3 \mathrm{H})$, 3.79 (s, 3H). ${ }^{13} \mathbf{C}$ NMR $\left(101 \mathrm{MHz}, \mathrm{CDCl}_{3}\right) \delta 165.2,155.3,153.3,138.5,133.0,129.2$, $129.0,126.8,126.7,121.4,115.9,113.8,110.0,88.7,55.8,52.8$. HRMS (ESI/TOF-Q) $\mathrm{m} / \mathrm{z}$ : $[\mathrm{M}+\mathrm{H}]^{+}$Calcd for $\mathrm{C}_{18} \mathrm{H}_{17} \mathrm{NO}_{4} \mathrm{H}^{+} 312.1230$; Found 312.1235.

Compound 4e was prepared following the general procedure for synthesis of 1,3Benzoxazepines described above by using $1 \mathrm{e}(0.22 \mathrm{mmol}, 52 \mathrm{mg})$ and purified by silica gel column chromatography $(1 \rightarrow 5 \%$ EtOAc : hexane) to provide pure compound as yellow solid $(66 \mathrm{mg}, 92 \%)$, melting point $=122-124{ }^{\circ} \mathrm{C} .{ }^{1} \mathbf{H}$ NMR $\left(400 \mathrm{MHz}, \mathrm{CDCl}_{3}\right) \delta$ $7.59-7.57(\mathrm{~m}, 4 \mathrm{H}), 7.52(\mathrm{bs}, 1 \mathrm{H}), 7.49-7.42(\mathrm{~m}, 5 \mathrm{H}), 7.37-7.32(\mathrm{~m}, 2 \mathrm{H}), 7.06(\mathrm{~d}, J=$ $8.3 \mathrm{~Hz}, 1 \mathrm{H}), 6.70(\mathrm{~s}, 1 \mathrm{H}), 5.67(\mathrm{~s}, 1 \mathrm{H}), 5.58(\mathrm{~d}, J=4.5 \mathrm{~Hz}, 1 \mathrm{H}), 3.87(\mathrm{~s}, 3 \mathrm{H}) .{ }^{13} \mathbf{C}$ NMR $\left(101 \mathrm{MHz}, \mathrm{CDCl}_{3}\right) \delta 165.1,158.6,140.5,138.4,136.2,133.1,131.1,129.3,129.0,128.9$, 127.2, 127.0, 126.7, 126.5, 126.1, 121.1, 110.6, 88.6, 52.9. HRMS (ESI/TOF-Q) m/z: $[\mathrm{M}+\mathrm{Na}]^{+} \mathrm{Calcd}$ for $\mathrm{C}_{23} \mathrm{H}_{19} \mathrm{NO}_{3} \mathrm{Na}^{+} 380.1257$; Found 380.1262.

Compound 4f was prepared following the general procedure for synthesis of 1,3Benzoxazepines described above by using $\mathbf{1 f}(0.22 \mathrm{mmol}, 43 \mathrm{mg})$ and purified by silica gel column chromatography $(1 \rightarrow 5 \%$ EtOAc : hexane) to provide pure compound as yellow solid $(50 \mathrm{mg}, 80 \%)$, melting point $=106-108{ }^{\circ} \mathrm{C} .{ }^{1} \mathbf{H}$ NMR $\left(400 \mathrm{MHz}, \mathrm{CDCl}_{3}\right) \delta$ $7.54-7.53(\mathrm{~m}, 2 \mathrm{H}), 7.45-7.44(\mathrm{~m}, 3 \mathrm{H}), 7.25(\mathrm{~s}, 1 \mathrm{H}), 7.04(\mathrm{~d}, J=8.6 \mathrm{~Hz}, 1 \mathrm{H}), 6.89(\mathrm{~d}, J$ $=8.6 \mathrm{~Hz}, 1 \mathrm{H}), 6.49(\mathrm{~s}, 1 \mathrm{H}), 5.72(\mathrm{~s}, 1 \mathrm{H}), 5.47(\mathrm{~d}, J=4.5 \mathrm{~Hz}, 1 \mathrm{H}), 3.85(\mathrm{~s}, 3 \mathrm{H}) .{ }^{13} \mathbf{C}$ NMR $\left(101 \mathrm{MHz}, \mathrm{CDCl}_{3}\right) \delta 164.7,157.4,137.9,133.6,131.2,129.3,128.9,128.0,127.6,127.1$, 126.6, 121.8, 108.6, 88.5, 52.8. HRMS (ESI/TOF-Q) $\mathrm{m} / \mathrm{z}:[\mathrm{M}+\mathrm{H}]^{+}$Calcd for $\mathrm{C}_{17} \mathrm{H}_{14} \mathrm{ClNO}_{3} \mathrm{H}^{+} 316.0735$; Found 316.0736. 

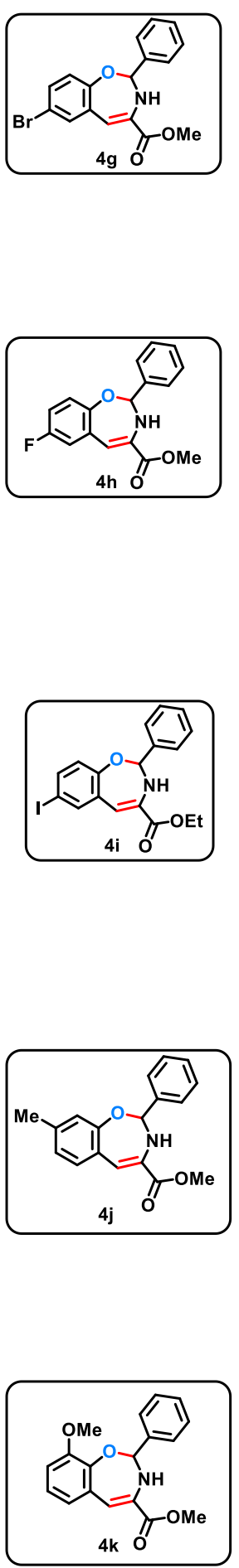

Compound $\mathbf{4 g}$ was prepared following the general procedure for synthesis of 1,3Benzoxazepines described above by using $1 \mathrm{~g}(0.22 \mathrm{mmol}, 52 \mathrm{mg})$ and purified by silica gel column chromatography $(1 \rightarrow 5 \%$ EtOAc : hexane $)$ to provide pure compound as yellow solid (56 mg, 78\%), melting point $=108-110{ }^{\circ} \mathrm{C} .{ }^{1} \mathbf{H}$ NMR $\left(400 \mathrm{MHz}, \mathrm{CDCl}_{3}\right) \delta$ $7.55-7.53(\mathrm{~m}, 2 \mathrm{H}), 7.46-7.45(\mathrm{~m}, 3 \mathrm{H}), 7.41(\mathrm{~d}, J=1.9 \mathrm{~Hz}, 1 \mathrm{H}), 7.19-7.17(\mathrm{~m}, 1 \mathrm{H})$, $6.85(\mathrm{~d}, J=8.6 \mathrm{~Hz}, 1 \mathrm{H}), 6.50(\mathrm{~s}, 1 \mathrm{H}), 5.74(\mathrm{~s}, 1 \mathrm{H}), 5.48(\mathrm{~d}, J=4.6 \mathrm{~Hz}, 1 \mathrm{H}), 3.85(\mathrm{~s}, 3 \mathrm{H})$. ${ }^{13}$ C NMR $\left(101 \mathrm{MHz}, \mathrm{CDCl}_{3}\right) \delta 164.8,158.0,137.9,134.3,133.6,130.1,129.4,129.0$, 128.2, 126.7, 122.3, 115.5, 108.6, 88.6, 52.9. HRMS (ESI/TOF-Q) m/z: $[\mathrm{M}+\mathrm{Na}]^{+}$Calcd for $\mathrm{C}_{17} \mathrm{H}_{14} \mathrm{BrNO}_{3} \mathrm{Na}^{+} 382.0049$; Found 382.0051 .

Compound 4h was prepared following the general procedure for synthesis of 1,3Benzoxazepines described above by using $1 \mathbf{h}(0.22 \mathrm{mmol}, 40 \mathrm{mg})$ and purified by silica gel column chromatography $(1 \rightarrow 5 \%$ EtOAc : hexane) to provide pure compound as yellow solid (46 mg, 77\%), melting point $=90-92{ }^{\circ} \mathrm{C} .{ }^{1} \mathbf{H}$ NMR $\left(400 \mathrm{MHz}, \mathrm{CDCl}_{3}\right) \delta$ $7.56-7.54(\mathrm{~m}, 2 \mathrm{H}), 7.46-7.45(\mathrm{~m}, 3 \mathrm{H}), 6.98-6.90(\mathrm{~m}, 2 \mathrm{H}), 6.82-6.78(\mathrm{~m}, 1 \mathrm{H}), 6.51$ $(\mathrm{s}, 1 \mathrm{H}), 5.73(\mathrm{~s}, 1 \mathrm{H}), 5.49(\mathrm{~d}, J=4.6 \mathrm{~Hz}, 1 \mathrm{H}), 3.86(\mathrm{~s}, 3 \mathrm{H}) .{ }^{13} \mathbf{C} \mathbf{~ N M R}\left(101 \mathrm{MHz}, \mathrm{CDCl}_{3}\right)$ $\delta 164.9,157.5(\mathrm{~d}, J=462.0 \mathrm{~Hz}), 155.2,138.1,133.6,129.3,129.0,127.6(\mathrm{~d}, J=8.3 \mathrm{~Hz})$, 126.7, $121.7(\mathrm{~d}, J=8.7 \mathrm{~Hz}), 117.4(\mathrm{~d}, J=23.6 \mathrm{~Hz}), 114.1(\mathrm{~d}, J=23.4 \mathrm{~Hz}), 108.7,88.7$, 52.9. ${ }^{19} \mathbf{F}$ NMR $\left(471 \mathrm{MHz}, \mathrm{CDCl}_{3}\right) \delta-121.75$ (s). HRMS (ESI/TOF-Q) m/z: $[\mathrm{M}+\mathrm{H}]^{+}$ Calcd for $\mathrm{C}_{17} \mathrm{H}_{14} \mathrm{FNO}_{3} \mathrm{H}^{+} 300.1030$; Found 300.1040.

Compound 4i was prepared following the general procedure for synthesis of 1,3Benzoxazepines described above by using $1 \mathbf{i}(0.22 \mathrm{mmol}, 62 \mathrm{mg})$ and purified by silica gel column chromatography $(1 \rightarrow 5 \%$ EtOAc : hexane) to provide pure compound as yellow solid $(64 \mathrm{mg}, 79 \%)$, melting point $=98-100{ }^{\circ} \mathrm{C} .{ }^{1} \mathbf{H}$ NMR $\left(400 \mathrm{MHz}, \mathrm{CDCl}_{3}\right) \delta$ $7.60(\mathrm{~d}, J=1.7 \mathrm{~Hz}, 1 \mathrm{H}), 7.55-7.53(\mathrm{~m}, 2 \mathrm{H}), 7.47-7.43(\mathrm{~m}, 3 \mathrm{H}), 7.37-7.35(\mathrm{~m}, 1 \mathrm{H})$, $6.73(\mathrm{~d}, J=8.5 \mathrm{~Hz}, 1 \mathrm{H}), 6.48(\mathrm{~s}, 1 \mathrm{H}), 5.73(\mathrm{~s}, 1 \mathrm{H}), 5.47(\mathrm{~d}, J=4.6 \mathrm{~Hz}, 1 \mathrm{H}), 3.85(\mathrm{~s}, 3 \mathrm{H})$. ${ }^{13}$ C NMR $\left(101 \mathrm{MHz}, \mathrm{CDCl}_{3}\right) \delta 164.7,158.8,140.4,137.9,136.0,133.5,129.4,129.0$, 128.6, 126.7, 122.7, 108.6, 88.6, 86.0, 52.9. HRMS (ESI/TOF-Q) m/z: [M+Na] ${ }^{+}$Calcd for $\mathrm{C}_{17} \mathrm{H}_{14} \mathrm{INO}_{3} \mathrm{Na}^{+} 429.9911$; Found 429.9918 .

Compound $\mathbf{4} \mathbf{j}$ was prepared following the general procedure for synthesis of 1,3Benzoxazepines described above by using $\mathbf{1 j}(0.22 \mathrm{mmol}, 39 \mathrm{mg})$ and purified by silica gel column chromatography ( $\rightarrow 5 \%$ EtOAc : hexane) to provide pure compound as yellow solid $(50 \mathrm{mg}, 85 \%)$, melting point $=104-106{ }^{\circ} \mathrm{C} .{ }^{1} \mathbf{H}$ NMR $\left(400 \mathrm{MHz}, \mathrm{CDCl}_{3}\right) \delta$ $7.57-7.55(\mathrm{~m}, 2 \mathrm{H}), 7.48-7.42(\mathrm{~m}, 3 \mathrm{H}), 7.18(\mathrm{~d}, J=7.7 \mathrm{~Hz}, 1 \mathrm{H}), 6.86-6.84(\mathrm{~m}, 2 \mathrm{H})$, $6.62(\mathrm{~s}, 1 \mathrm{H}), 5.55(\mathrm{~s}, 1 \mathrm{H}), 5.53(\mathrm{~d}, J=4.5 \mathrm{~Hz}, 1 \mathrm{H}), 3.85(\mathrm{~s}, 3 \mathrm{H}), 2.29(\mathrm{~s}, 3 \mathrm{H}) .{ }^{13} \mathbf{C}$ NMR $\left(101 \mathrm{MHz}, \mathrm{CDCl}_{3}\right) \delta 165.3,158.9,138.6,138.4,132.4,132.1,129.2,129.0,126.7,124.1$, 122.9, 121.3, 111.0, 88.4, 52.7, 21.1. HRMS (ESI/TOF-Q) $\mathrm{m} / \mathrm{z}:[\mathrm{M}+\mathrm{H}]^{+}$Calcd for $\mathrm{C}_{18} \mathrm{H}_{17} \mathrm{NO}_{3} \mathrm{H}^{+} 296.1281$; Found 296.1285

Compound 4k was prepared following the general procedure for synthesis of 1,3Benzoxazepines described above by using $1 \mathbf{k}(0.22 \mathrm{mmol}, 43 \mathrm{mg})$ and purified by silica gel column chromatography ( $\rightarrow 5 \%$ EtOAc : hexane) to provide pure compound as yellow solid $(43 \mathrm{mg}, 69 \%)$, melting point $=128-130{ }^{\circ} \mathrm{C} .{ }^{1} \mathbf{H}$ NMR $\left(400 \mathrm{MHz}, \mathrm{CDCl}_{3}\right) \delta$ $7.66(\mathrm{~d}, J=6.6 \mathrm{~Hz}, 2 \mathrm{H}), 7.45-7.41(\mathrm{~m}, 3 \mathrm{H}), 6.98-6.89(\mathrm{~m}, 2 \mathrm{H}), 6.79-6.77(\mathrm{~m}, 1 \mathrm{H})$, $6.62(\mathrm{~s}, 1 \mathrm{H}), 5.69(\mathrm{~s}, 1 \mathrm{H}), 5.57(\mathrm{~d}, J=5.3 \mathrm{~Hz}, 1 \mathrm{H}), 3.86(\mathrm{~s}, 3 \mathrm{H}), 3.80(\mathrm{~s}, 3 \mathrm{H}) .{ }^{13} \mathbf{C}$ NMR $\left(101 \mathrm{MHz}, \mathrm{CDCl}_{3}\right) \delta 165.2,151.2,149.0,138.3,132.7,129.0,128.9,127.4,126.7,124.4$, 123.0, 111.1, 110.7, 89.2, 56.6, 52.8. HRMS (ESI/TOF-Q) m/z: $[\mathrm{M}+\mathrm{Na}]^{+}$Calcd for $\mathrm{C}_{18} \mathrm{H}_{17} \mathrm{NO}_{4} \mathrm{Na}^{+} 334.1050$; Found 334.1064. 


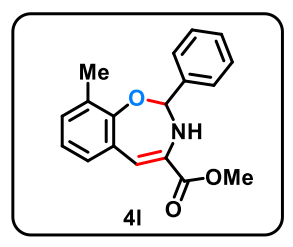

Compound 4l was prepared following the general procedure for synthesis of 1,3Benzoxazepines described above by using $11(0.22 \mathrm{mmol}, 39 \mathrm{mg})$ and purified by silica gel column chromatography $(1 \rightarrow 5 \%$ EtOAc : hexane) to provide pure compound as yellow solid $(40 \mathrm{mg}, 67 \%)$, melting point $=82-84{ }^{\circ} \mathrm{C} .{ }^{1} \mathbf{H}$ NMR $\left(400 \mathrm{MHz}, \mathrm{CDCl}_{3}\right) \delta$ $7.61(\mathrm{~d}, J=7.6 \mathrm{~Hz}, 2 \mathrm{H}), 7.48-7.42(\mathrm{~m}, 3 \mathrm{H}), 7.15(\mathrm{~d}, J=7.7 \mathrm{~Hz}, 1 \mathrm{H}), 7.03(\mathrm{~d}, J=7.3$ $\mathrm{Hz}, 1 \mathrm{H}), 6.95-6.91(\mathrm{~m}, 1 \mathrm{H}), 6.63(\mathrm{~s}, 1 \mathrm{H}), 5.60(\mathrm{~s}, 1 \mathrm{H}), 5.55(\mathrm{~d}, J=4.9 \mathrm{~Hz}, 1 \mathrm{H}), 3.86(\mathrm{~s}$, $3 \mathrm{H}), 2.21(\mathrm{~s}, 3 \mathrm{H}) .{ }^{13} \mathbf{C}$ NMR $\left(101 \mathrm{MHz}, \mathrm{CDCl}_{3}\right) \delta 165.3,157.6,138.7,132.4,130.4$, $129.5,129.2,129.1,128.9,126.5,125.9,122.8,110.9,88.5,52.8,16.9$. HRMS (ESI/TOF-Q) m/z: [M+Na] ${ }^{+}$Calcd for $\mathrm{C}_{18} \mathrm{H}_{17} \mathrm{NO}_{3} \mathrm{Na}^{+} 318.1101$; Found 318.1107.

Compound $\mathbf{4 m}$ was prepared following the general procedure for synthesis of 1,3Benzoxazepines described above by using $1 \mathrm{~m}(0.22 \mathrm{mmol}, 52 \mathrm{mg})$ and purified by silica gel column chromatography $(1 \rightarrow 5 \%$ EtOAc : hexane $)$ to provide pure compound as yellow solid $(50 \mathrm{mg}, 70 \%)$, melting point $=118-120^{\circ} \mathrm{C} .{ }^{1} \mathbf{H}$ NMR $\left(400 \mathrm{MHz}, \mathrm{CDCl}_{3}\right) \delta$ $7.39-7.36(\mathrm{~m}, 2 \mathrm{H}), 7.32-7.30(\mathrm{~m}, 6 \mathrm{H}), 7.25(\mathrm{bs}, 3 \mathrm{H}), 7.20(\mathrm{~d}, J=7.4 \mathrm{~Hz}, 1 \mathrm{H}), 7.11-$ $7.07(\mathrm{~m}, 1 \mathrm{H}), 6.69(\mathrm{~s}, 1 \mathrm{H}), 5.64(\mathrm{~d}, J=5.7 \mathrm{~Hz}, 1 \mathrm{H}), 5.61(\mathrm{~s}, 1 \mathrm{H}), 3.86(\mathrm{~s}, 3 \mathrm{H}) .{ }^{13} \mathbf{C}$ NMR $\left(101 \mathrm{MHz}, \mathrm{CDCl}_{3}\right) \delta 165.2,156.4,138.4,137.6,134.0,132.6,131.9,130.0,129.1,128.8$, 128.6, 127.8, 127.4, 127.0, 126.7, 123.4, 110.8, 89.3, 52.8. HRMS (ESI/TOF-Q) m/z: $[\mathrm{M}+\mathrm{Na}]^{+}$Calcd for $\mathrm{C}_{23} \mathrm{H}_{19} \mathrm{NO}_{3} \mathrm{Na}^{+} 380.1257$; Found 380.1262.

Compound 4n was prepared following the general procedure for synthesis of 1,3Benzoxazepines described above by using $1 \mathrm{n}(0.22 \mathrm{mmol}, 42 \mathrm{mg})$ and purified by silica gel column chromatography ( $\rightarrow 5 \%$ EtOAc : hexane) to provide pure compound as yellow solid $(38 \mathrm{mg}, 61 \%)$, melting point $=84-86{ }^{\circ} \mathrm{C} .{ }^{1} \mathbf{H}$ NMR $\left(400 \mathrm{MHz}, \mathrm{CDCl}_{3}\right) \delta$ $7.61(\mathrm{~d}, J=7.9 \mathrm{~Hz}, 2 \mathrm{H}), 7.47-7.42(\mathrm{~m}, 3 \mathrm{H}), 6.92-6.90(\mathrm{~m}, 1 \mathrm{H}), 6.84-6.82(\mathrm{~m}, J=8.4$ $\mathrm{Hz}, 2 \mathrm{H}), 5.63(\mathrm{~s}, 1 \mathrm{H}), 5.57(\mathrm{~d}, J=5.5 \mathrm{~Hz}, 1 \mathrm{H}), 3.86(\mathrm{~s}, 3 \mathrm{H}), 2.45(\mathrm{~s}, 3 \mathrm{H}), 2.15(\mathrm{~s}, 3 \mathrm{H})$. ${ }^{13}$ C NMR $\left(101 \mathrm{MHz}, \mathrm{CDCl}_{3}\right) \delta 165.5,159.0,138.8,136.1,132.1,129.1,128.9,128.5$, 127.5, 126.5, 125.6, 125.1, 106.2, 89.5, 52.8, 20.8, 16.7. HRMS (ESI/TOF-Q) m/z: $[\mathrm{M}+\mathrm{H}]^{+}$Calcd for $\mathrm{C}_{19} \mathrm{H}_{19} \mathrm{NO}_{3} \mathrm{H}^{+} 310.1438$; Found 310.1443.

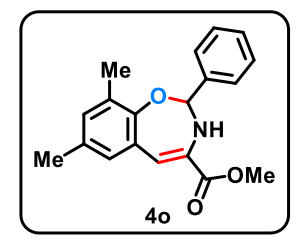

Compound 4o was prepared following the general procedure for synthesis of 1,3Benzoxazepines described above by using $10(0.22 \mathrm{mmol}, 42 \mathrm{mg})$ and purified by silica gel column chromatography ( $\rightarrow 5 \%$ EtOAc : hexane) to provide pure compound as yellow solid $(40 \mathrm{mg}, 65 \%)$, melting point $=88-90{ }^{\circ} \mathrm{C} .{ }^{1} \mathbf{H}$ NMR $\left(400 \mathrm{MHz}, \mathrm{CDCl}_{3}\right) \delta$ $7.61(\mathrm{~d}, J=7.3 \mathrm{~Hz}, 2 \mathrm{H}), 7.47-7.42(\mathrm{~m}, 3 \mathrm{H}), 6.95(\mathrm{~s}, 1 \mathrm{H}), 6.85(\mathrm{~s}, 1 \mathrm{H}), 6.59(\mathrm{~s}, 1 \mathrm{H})$, $5.57(\mathrm{~s}, 1 \mathrm{H}), 5.53(\mathrm{~d}, J=4.8 \mathrm{~Hz}, 1 \mathrm{H}), 3.85(\mathrm{~s}, 3 \mathrm{H}), 2.27(\mathrm{~s}, 3 \mathrm{H}), 2.17(\mathrm{~s}, 3 \mathrm{H}) \cdot{ }^{13} \mathbf{C}$ NMR $\left(101 \mathrm{MHz}, \mathrm{CDCl}_{3}\right) \delta 165.3,155.5,138.8,132.4,132.0,130.6,130.2,129.2,129.0,128.9$, 126.5, 125.6, 110.9, 88.5, 52.7, 20.6, 16.8. HRMS (ESI/TOF-Q) m/z: $[\mathrm{M}+\mathrm{H}]^{+}$Calcd for $\mathrm{C}_{19} \mathrm{H}_{19} \mathrm{NO}_{3} \mathrm{H}^{+} 310.1438$; Found 310.1403.

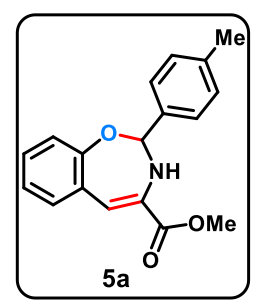

Compound 5a was prepared following the general procedure for synthesis of 1,3Benzoxazepines described above by using $1 \mathrm{a}(0.22 \mathrm{mmol}, 36 \mathrm{mg})$ and purified by silica gel column chromatography $(1 \rightarrow 5 \%$ EtOAc : hexane) to provide pure compound as yellow solid $(50 \mathrm{mg}, 84 \%)$, melting point $=108-110^{\circ} \mathrm{C}$. ${ }^{1} \mathbf{H}$ NMR $\left(400 \mathrm{MHz}, \mathrm{CDCl}_{3}\right) \delta$ $7.44(\mathrm{~d}, J=7.7 \mathrm{~Hz}, 2 \mathrm{H}), 7.29-7.25(\mathrm{~m}, 3 \mathrm{H}), 7.13-7.09(\mathrm{~m}, 1 \mathrm{H}), 7.03-6.96(\mathrm{~m}, 2 \mathrm{H})$, $6.60(\mathrm{~s}, 1 \mathrm{H}), 5.61(\mathrm{~s}, 1 \mathrm{H}), 5.49(\mathrm{~d}, J=4.5 \mathrm{~Hz}, 1 \mathrm{H}), 3.84(\mathrm{~s}, 3 \mathrm{H}), 2.40(\mathrm{~s}, 3 \mathrm{H}) .{ }^{13} \mathbf{C}$ NMR $\left(101 \mathrm{MHz}, \mathrm{CDCl}_{3}\right) \delta 165.2,159.1,139.1,135.6,132.7,132.5,129.6,127.7,126.6,125.9$, 123.1, 120.7, 110.4, 88.4, 52.8, 21.4. HRMS (ESI/TOF-Q) $\mathrm{m} / \mathrm{z}:[\mathrm{M}+\mathrm{H}]^{+}$Calcd for $\mathrm{C}_{18} \mathrm{H}_{17} \mathrm{NO}_{3} \mathrm{H}^{+} 296.1281$; Found 296.1287. 

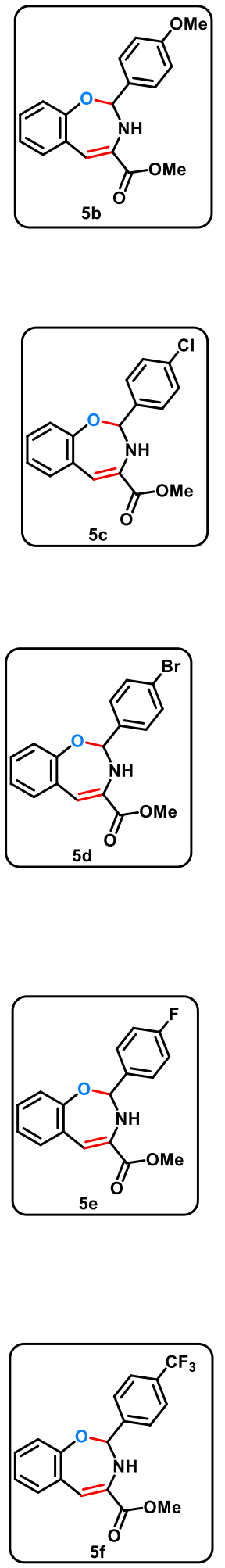

Compound $\mathbf{5 b}$ was prepared following the general procedure for synthesis of 1,3Benzoxazepines described above by using $1 \mathrm{a}(0.22 \mathrm{mmol}, 36 \mathrm{mg})$ and purified by silica gel column chromatography $(1 \rightarrow 5 \%$ EtOAc : hexane) to provide pure compound as yellow solid $(55 \mathrm{mg}, 88 \%)$, melting point $=96-98{ }^{\circ} \mathrm{C} .{ }^{1} \mathbf{H}$ NMR $\left(400 \mathrm{MHz}, \mathrm{CDCl}_{3}\right) \delta$ $7.55(\mathrm{~d}, J=7.4 \mathrm{~Hz}, 2 \mathrm{H}), 7.47-7.42(\mathrm{~m}, 3 \mathrm{H}), 6.90(\mathrm{~d}, J=8.8 \mathrm{~Hz}, 1 \mathrm{H}), 6.79(\mathrm{~d}, J=2.5$ $\mathrm{Hz}, 1 \mathrm{H}), 6.70-6.67(\mathrm{~m}, 1 \mathrm{H}), 6.56(\mathrm{~s}, 1 \mathrm{H}), 5.63(\mathrm{~s}, 1 \mathrm{H}), 5.49(\mathrm{~d}, J=4.6 \mathrm{~Hz}, 1 \mathrm{H}), 3.85(\mathrm{~s}$, $3 \mathrm{H}), 3.79(\mathrm{~s}, 3 \mathrm{H}) .{ }^{13} \mathbf{C}$ NMR $\left(101 \mathrm{MHz}, \mathrm{CDCl}_{3}\right) \delta 165.2,155.3,153.3,138.5,133.0$, $129.2,129.0,126.8,126.7,121.4,115.9,113.8,110.0,88.7,55.8,52.8$. HRMS (ESI/TOF-Q) m/z: [M+H] $]^{+}$Calcd for $\mathrm{C}_{18} \mathrm{H}_{17} \mathrm{NO}_{4} \mathrm{H}^{+} 312.1230$; Found 312.1240 .

Compound 5c was prepared following the general procedure for synthesis of 1,3Benzoxazepines described above by using $1 \mathrm{a}(0.22 \mathrm{mmol}, 36 \mathrm{mg})$ and purified by silica gel column chromatography $(1 \rightarrow 5 \%$ EtOAc : hexane) to provide pure compound as yellow solid $(50 \mathrm{mg}, 79 \%)$, melting point $=99-101{ }^{\circ} \mathrm{C} .{ }^{1} \mathbf{H}$ NMR $\left(400 \mathrm{MHz}, \mathrm{CDCl}_{3}\right) \delta$ $7.55(\mathrm{~d}, J=6.2 \mathrm{~Hz}, 2 \mathrm{H}), 7.47-7.45(\mathrm{~m}, 3 \mathrm{H}), 7.26(\mathrm{~s}, 1 \mathrm{H}), 7.05(\mathrm{~d}, J=8.5 \mathrm{~Hz}, 1 \mathrm{H}), 6.92$ - $6.90(\mathrm{~m}, 1 \mathrm{H}), 6.51(\mathrm{~s}, 1 \mathrm{H}), 5.73(\mathrm{~s}, 1 \mathrm{H}), 5.48(\mathrm{~d}, J=4.4 \mathrm{~Hz}, 1 \mathrm{H}), 3.86(\mathrm{~s}, 3 \mathrm{H}) .{ }^{13} \mathrm{C}$ NMR $\left(101 \mathrm{MHz}, \mathrm{CDCl}_{3}\right) \delta 164.8,157.6,138.0,133.7,131.4,129.4,129.0,128.0,127.7$, 127.2, 126.7, 122.0, 108.7, 88.7, 52.9. HRMS (ESI/TOF-Q) m/z: $[\mathrm{M}+\mathrm{H}]^{+}$Calcd for $\mathrm{C}_{17} \mathrm{H}_{14} \mathrm{ClNO}_{3} \mathrm{H}^{+} 316.0735$; Found 316.0740.

Compound 5d was prepared following the general procedure for synthesis of 1,3Benzoxazepines described above by using $1 \mathbf{a}(0.22 \mathrm{mmol}, 36 \mathrm{mg})$ and purified by silica gel column chromatography $(1 \rightarrow 5 \%$ EtOAc : hexane $)$ to provide pure compound as yellow solid (55 mg, 77\%), melting point $=108-110^{\circ} \mathrm{C} .{ }^{1} \mathbf{H}$ NMR $\left(400 \mathrm{MHz}, \mathrm{CDCl}_{3}\right) \delta$ $7.59(\mathrm{~d}, J=7.9 \mathrm{~Hz}, 2 \mathrm{H}), 7.44(\mathrm{~d}, J=7.9 \mathrm{~Hz}, 2 \mathrm{H}), 7.29-7.26(\mathrm{~m}, 1 \mathrm{H}), 7.15-7.11(\mathrm{~m}$, $1 \mathrm{H}), 7.05-7.02(\mathrm{~m}, 1 \mathrm{H}), 6.97(\mathrm{~d}, J=8.1 \mathrm{~Hz}, 1 \mathrm{H}), 6.62(\mathrm{~s}, 1 \mathrm{H}), 5.54(\mathrm{~s}, 1 \mathrm{H}), 5.50(\mathrm{~d}, J=$ $3.9 \mathrm{~Hz}, 1 \mathrm{H}), 3.86(\mathrm{~s}, 3 \mathrm{H}) .{ }^{13} \mathrm{C}$ NMR $\left(101 \mathrm{MHz}, \mathrm{CDCl}_{3}\right) \delta 165.0,158.8,137.4,132.6$, $132.6,132.1,128.5,127.9,125.8,123.4(2 \times \mathrm{C}), 120.7,110.9,87.8,52.9$. HRMS (ESI/TOF-Q) m/z: [M+Na] ${ }^{+}$Calcd for $\mathrm{C}_{17} \mathrm{H}_{14} \mathrm{BrNO}_{3} \mathrm{Na}^{+} 382.0049$; Found 382.0054 .

Compound 5e was prepared following the general procedure for synthesis of 1,3Benzoxazepines described above by using $1 \mathrm{a}(0.22 \mathrm{mmol}, 36 \mathrm{mg})$ and purified by silica gel column chromatography $(1 \rightarrow 5 \%$ EtOAc : hexane $)$ to provide pure compound as yellow solid $(45 \mathrm{mg}, 75 \%)$, melting point $=98-100{ }^{\circ} \mathrm{C} .{ }^{1} \mathbf{H}$ NMR $\left(400 \mathrm{MHz}, \mathrm{CDCl}_{3}\right) \delta$ $7.56-7.52(\mathrm{~m}, 2 \mathrm{H}), 7.29(\mathrm{~d}, J=7.6 \mathrm{~Hz}, 1 \mathrm{H}), 7.16-7.11(\mathrm{~m}, 3 \mathrm{H}), 7.07-7.01(\mathrm{~m}, 1 \mathrm{H})$, $6.98-6.96(\mathrm{~m}, 1 \mathrm{H}), 6.62(\mathrm{~s}, 1 \mathrm{H}), 5.56(\mathrm{~s}, 1 \mathrm{H}), 5.52(\mathrm{~d}, J=4.3 \mathrm{~Hz}, 1 \mathrm{H}), 3.86(\mathrm{~s}, 3 \mathrm{H}) .{ }^{13} \mathbf{C}$ NMR $\left(101 \mathrm{MHz}, \mathrm{CDCl}_{3}\right) \delta 165.2,163.2(\mathrm{~d}, J=247.8 \mathrm{~Hz}), 158.9,134.4(\mathrm{~d}, J=3.3 \mathrm{~Hz})$, $132.6(2 \mathrm{xC}), 128.6(\mathrm{~d}, J=8.4 \mathrm{~Hz}), 127.9,125.8,123.3,120.7,115.9(\mathrm{~d}, J=21.7 \mathrm{~Hz})$, $110.8,87.8,52.9 .{ }^{19} \mathbf{F}$ NMR $\left(471 \mathrm{MHz}, \mathrm{CDCl}_{3}\right) \delta-112.45$ (s). HRMS (ESI/TOF-Q) m/z: $[\mathrm{M}+\mathrm{H}]^{+}$Calcd for $\mathrm{C}_{17} \mathrm{H}_{14} \mathrm{FNO}_{3} \mathrm{H}^{+} 300.1030$; Found 300.1051.

Compound 5f was prepared following the general procedure for synthesis of 1,3Benzoxazepines described above by using $1 \mathrm{a}(0.22 \mathrm{mmol}, 36 \mathrm{mg})$ and purified by silica gel column chromatography ( $\rightarrow 5 \%$ EtOAc : hexane) to provide pure compound as yellow solid (50 mg, 72\%), melting point $=138-140{ }^{\circ} \mathrm{C} .{ }^{1} \mathbf{H}$ NMR $\left(400 \mathrm{MHz}, \mathrm{CDCl}_{3}\right) \delta$ $7.74-7.69(\mathrm{~m}, 4 \mathrm{H}), 7.30(\mathrm{~d}, J=7.6 \mathrm{~Hz}, 1 \mathrm{H}), 7.14-7.12(\mathrm{~m}, 1 \mathrm{H}), 7.06-7.04(\mathrm{~m}, 1 \mathrm{H})$, $6.99(\mathrm{~d}, J=8.0 \mathrm{~Hz}, 1 \mathrm{H}), 6.65(\mathrm{~s}, 1 \mathrm{H}), 5.60(\mathrm{~d}, J=4.8 \mathrm{~Hz}, 1 \mathrm{H}), 5.57(\mathrm{~s}, 1 \mathrm{H}), 3.86(\mathrm{~s}, 3 \mathrm{H})$. ${ }^{13}$ C NMR $\left(101 \mathrm{MHz}, \mathrm{CDCl}_{3}\right) \delta 165.0,158.8,142.0,132.7,132.7,128.0,127.3(2 \mathrm{xC})$, $126.0(\mathrm{q}, J=3.6 \mathrm{~Hz}), 125.8,125.8,123.5,120.7,111.2,87.7,52.9 .{ }^{19} \mathbf{F}$ NMR $(471 \mathrm{MHz}$, $\left.\mathrm{CDCl}_{3}\right) \delta-62.69(\mathrm{~s})$. HRMS (ESI/TOF-Q) $\mathrm{m} / \mathrm{z}:[\mathrm{M}+\mathrm{H}]^{+}$Calcd for $\mathrm{C}_{18} \mathrm{H}_{14} \mathrm{~F}_{3} \mathrm{NO}_{3} \mathrm{H}^{+}$ 350.0999 ; Found 350.1003. 


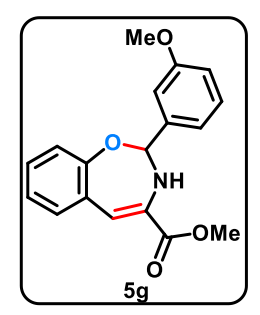

Compound 5g was prepared following the general procedure for synthesis of $1,3-$ Benzoxazepines described above by using $1 \mathbf{a}(0.22 \mathrm{mmol}, 36 \mathrm{mg})$ and purified by silica gel column chromatography $(1 \rightarrow 5 \%$ EtOAc : hexane) to provide pure compound as yellow solid (54 mg, 86\%), melting point $=112-114^{\circ} \mathrm{C} .{ }^{1} \mathbf{H}$ NMR $\left(400 \mathrm{MHz}, \mathrm{CDCl}_{3}\right) \delta$ $7.36-7.32(\mathrm{~m}, 1 \mathrm{H}), 7.28-7.23(\mathrm{~m}, 1 \mathrm{H}), 7.11-7.09(\mathrm{~m}, 3 \mathrm{H}), 7.02-6.94(\mathrm{~m}, 3 \mathrm{H}), 6.60$ $(\mathrm{s}, 1 \mathrm{H}), 5.62(\mathrm{~s}, 1 \mathrm{H}), 5.47(\mathrm{~d}, J=4.2 \mathrm{~Hz}, 1 \mathrm{H}), 3.83(\mathrm{~s}, 6 \mathrm{H}) .{ }^{13} \mathbf{C}$ NMR $\left(101 \mathrm{MHz}, \mathrm{CDCl}_{3}\right)$ $\delta 165.1,160.1,158.9,139.8,132.7,132.5,130.0,127.8,125.9,123.2,120.7,118.9$, 114.9, 112.0, 110.5, 88.3, 55.5, 52.8. HRMS (ESI/TOF-Q) m/z: $[\mathrm{M}+\mathrm{Na}]^{+}$Calcd for $\mathrm{C}_{18} \mathrm{H}_{17} \mathrm{NO}_{4} \mathrm{Na}^{+} 334.1050$; Found 334.1015.

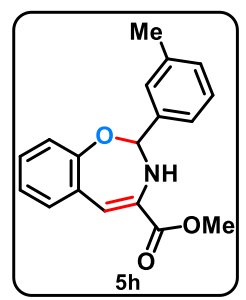

Compound $\mathbf{5 h}$ was prepared following the general procedure for synthesis of 1,3Benzoxazepines described above by using $1 \mathrm{a}(0.22 \mathrm{mmol}, 36 \mathrm{mg})$ and purified by silica gel column chromatography $(1 \rightarrow 5 \%$ EtOAc : hexane $)$ to provide pure compound as yellow solid (49 mg, 83\%), melting point $=113-115^{\circ} \mathrm{C} .{ }^{1} \mathbf{H}$ NMR $\left(400 \mathrm{MHz}, \mathrm{CDCl}_{3}\right) \delta$ $7.29-7.27(\mathrm{~m}, 3 \mathrm{H}), 7.25-7.24(\mathrm{~m}, 2 \mathrm{H}), 7.13-7.09(\mathrm{~m}, 1 \mathrm{H}), 7.03-6.97(\mathrm{~m}, 2 \mathrm{H}), 6.61$ (s, 1H), $5.62(\mathrm{~s}, 1 \mathrm{H}), 5.48(\mathrm{~d}, J=4.2 \mathrm{~Hz}, 1 \mathrm{H}), 3.85(\mathrm{~s}, 3 \mathrm{H}), 2.41(\mathrm{~s}, 3 \mathrm{H}) .{ }^{13} \mathbf{C}$ NMR $(101$ $\left.\mathrm{MHz}, \mathrm{CDCl}_{3}\right) \delta 165.2,159.1,138.9,138.4,132.8,132.5,130.0,128.9,127.7,127.3$, 126.0, 123.8, 123.2, 120.7, 110.5, 88.6, 52.8, 21.6. HRMS (ESI/TOF-Q) m/z: $[\mathrm{M}+\mathrm{H}]^{+}$ Calcd for $\mathrm{C}_{18} \mathrm{H}_{17} \mathrm{NO}_{3} \mathrm{H}^{+} 296.1281$; Found 296.1283.

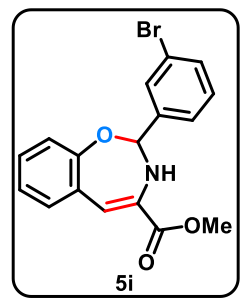

Compound 5i was prepared following the general procedure for synthesis of 1,3Benzoxazepines described above by using $1 \mathbf{a}(0.22 \mathrm{mmol}, 36 \mathrm{mg})$ and purified by silica gel column chromatography $(1 \rightarrow 5 \%$ EtOAc : hexane $)$ to provide pure compound as yellow solid $(58 \mathrm{mg}, 80 \%)$, melting point $=112-114{ }^{\circ} \mathrm{C} .{ }^{1} \mathbf{H}$ NMR $\left(400 \mathrm{MHz}, \mathrm{CDCl}_{3}\right) \delta$ $7.74(\mathrm{~d}, J=1.2 \mathrm{~Hz}, 1 \mathrm{H}), 7.55(\mathrm{~d}, J=8.0 \mathrm{~Hz}, 1 \mathrm{H}), 7.48(\mathrm{~d}, J=7.7 \mathrm{~Hz}, 1 \mathrm{H}), 7.34-$ $7.25(\mathrm{~m}, 2 \mathrm{H}), 7.15-7.11(\mathrm{~m}, J=7.6 \mathrm{~Hz}, 1 \mathrm{H}), 7.05-6.97(\mathrm{~m}, 2 \mathrm{H}), 6.63(\mathrm{~s}, 1 \mathrm{H}), 5.56(\mathrm{~s}$, $1 \mathrm{H}), 5.49(\mathrm{~d}, J=4.3 \mathrm{~Hz}, 1 \mathrm{H}), 3.86(\mathrm{~s}, 3 \mathrm{H}) .{ }^{13} \mathbf{C} \mathbf{~ N M R}\left(101 \mathrm{MHz}, \mathrm{CDCl}_{3}\right) \delta 165.1,158.8$, 140.4, 132.6, 132.6, 132.3, 130.5, 129.9, 127.9, 125.8, 125.4, 123.4, 123.0, 120.7, 111.0, 87.5, 52.9. HRMS (ESI/TOF-Q) m/z: $[\mathrm{M}+\mathrm{H}]^{+}$Calcd for $\mathrm{C}_{17} \mathrm{H}_{14} \mathrm{BrNO}_{3} \mathrm{H}^{+} 360.0230$; Found 360.0231 .

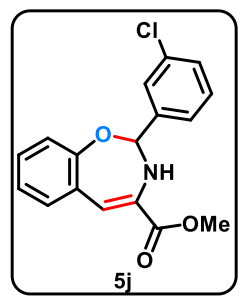

Compound 5j was prepared following the general procedure for synthesis of 1,3Benzoxazepines described above by using $1 \mathrm{a}(0.22 \mathrm{mmol}, 36 \mathrm{mg})$ and purified by silica gel column chromatography $(1 \rightarrow 5 \%$ EtOAc : hexane $)$ to provide pure compound as yellow solid (49 mg, 77\%), melting point $=98-100{ }^{\circ} \mathrm{C} .{ }^{1} \mathbf{H}$ NMR $\left(400 \mathrm{MHz}, \mathrm{CDCl}_{3}\right) \delta$ 7.59 (bs, 1H), $7.45-7.38(\mathrm{~m}, 3 \mathrm{H}), 7.30-7.28(\mathrm{~m}, 1 \mathrm{H}), 7.15-7.11(\mathrm{~m}, 1 \mathrm{H}), 7.05-6.98$ $(\mathrm{m}, 2 \mathrm{H}), 6.63(\mathrm{~s}, 1 \mathrm{H}), 5.57(\mathrm{~s}, 1 \mathrm{H}), 5.51(\mathrm{~d}, J=4.7 \mathrm{~Hz}, 1 \mathrm{H}), 3.86(\mathrm{~s}, 3 \mathrm{H}) .{ }^{13} \mathbf{C} \mathbf{N M R}(101$ $\left.\mathrm{MHz}, \mathrm{CDCl}_{3}\right) \delta 165.1,158.8,140.2,134.9,132.6(2 \mathrm{xC}), 130.3,129.4,127.9,127.0$, 125.8, 124.9, 123.4, 120.7, 111.0, 87.6, 52.9. HRMS (ESI/TOF-Q) m/z: $[\mathrm{M}+\mathrm{H}]^{+}$Calcd for $\mathrm{C}_{17} \mathrm{H}_{14} \mathrm{ClNO}_{3} \mathrm{H}^{+} 316.0735$; Found 316.0740.

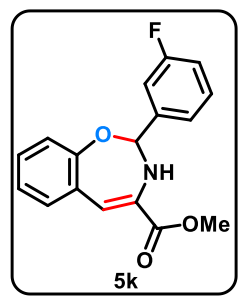

Compound 5k was prepared following the general procedure for synthesis of 1,3Benzoxazepines described above by using $1 \mathrm{a}(0.22 \mathrm{mmol}, 36 \mathrm{mg})$ and purified by silica gel column chromatography $(1 \rightarrow 5 \%$ EtOAc : hexane) to provide pure compound as yellow solid $(45 \mathrm{mg}, 76 \%)$, melting point $=111-113^{\circ} \mathrm{C} .{ }^{1} \mathbf{H}$ NMR $\left(400 \mathrm{MHz}, \mathrm{CDCl}_{3}\right) \delta$ $7.45-7.39(\mathrm{~m}, 1 \mathrm{H}), 7.34-7.28(\mathrm{~m}, 3 \mathrm{H}), 7.15-7.10(\mathrm{~m}, 2 \mathrm{H}), 7.06-6.99(\mathrm{~m}, 2 \mathrm{H}), 6.63$ $(\mathrm{s}, 1 \mathrm{H}), 5.58(\mathrm{~s}, 1 \mathrm{H}), 5.53(\mathrm{~d}, J=4.7 \mathrm{~Hz}, 1 \mathrm{H}), 3.86(\mathrm{~s}, 3 \mathrm{H}) .{ }^{13} \mathbf{C} \mathbf{N M R}\left(101 \mathrm{MHz}, \mathrm{CDCl}_{3}\right)$ $\delta 165.1,163.0(\mathrm{~d}, J=248 \mathrm{~Hz}), 158.8,140.6(\mathrm{~d}, J=7.3 \mathrm{~Hz}), 132.6,130.6(\mathrm{~d}, J=8.1 \mathrm{~Hz})$, $127.9(2 \mathrm{xC}), 125.8,123.4,122.4(\mathrm{~d}, J=3.2 \mathrm{~Hz}), 120.7,116.2(\mathrm{~d}, J=21.1 \mathrm{~Hz}), 114.0(\mathrm{~d}$, $J=22.7 \mathrm{~Hz}), 111.0,87.6,52.9 .{ }^{19} \mathbf{F}$ NMR $\left(471 \mathrm{MHz}, \mathrm{CDCl}_{3}\right) \delta-111.80$ (s). HRMS (ESI/TOF-Q) m/z: $[\mathrm{M}+\mathrm{H}]^{+}$Calcd for $\mathrm{C}_{17} \mathrm{H}_{14} \mathrm{FNO}_{3} \mathrm{H}^{+} 300.1030$; Found 300.1053 . 


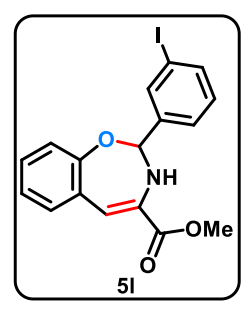

Compound $5 \mathbf{l}$ was prepared following the general procedure for synthesis of 1,3Benzoxazepines described above by using $1 \mathrm{a}(0.22 \mathrm{mmol}, 36 \mathrm{mg})$ and purified by silica gel column chromatography ( $\rightarrow 5 \%$ EtOAc : hexane) to provide pure compound as yellow solid (64 mg, 79\%), melting point $=118-120{ }^{\circ} \mathrm{C} .{ }^{1} \mathbf{H}$ NMR $\left(400 \mathrm{MHz}, \mathrm{CDCl}_{3}\right) \delta$ $7.93(\mathrm{~s}, 1 \mathrm{H}), 7.76(\mathrm{~d}, J=7.8 \mathrm{~Hz}, 1 \mathrm{H}), 7.52(\mathrm{~d}, J=7.6 \mathrm{~Hz}, 1 \mathrm{H}), 7.28(\mathrm{~d}, J=7.6 \mathrm{~Hz}, 1 \mathrm{H})$, $7.20-7.11(\mathrm{~m}, 2 \mathrm{H}), 7.05-6.98(\mathrm{~m}, 2 \mathrm{H}), 6.63(\mathrm{~s}, 1 \mathrm{H}), 5.55(\mathrm{~s}, 1 \mathrm{H}), 5.47(\mathrm{~d}, J=4.4 \mathrm{~Hz}$, $1 \mathrm{H}), 3.86(\mathrm{~s}, 3 \mathrm{H}) .{ }^{13} \mathrm{C}$ NMR $\left(101 \mathrm{MHz}, \mathrm{CDCl}_{3}\right) \delta 165.1,158.8,140.5,138.3,135.8$, 132.6 (2xC), 130.6, 127.9, 126.0, 125.8, 123.4, 120.7, 111.0, 94.6, 87.5, 52.9. HRMS (ESI/TOF-Q) m/z: $[\mathrm{M}+\mathrm{H}]^{+}$Calcd for $\mathrm{C}_{17} \mathrm{H}_{14} \mathrm{INO}_{3} \mathrm{H}^{+} 408.0091$; Found 408.0095 .

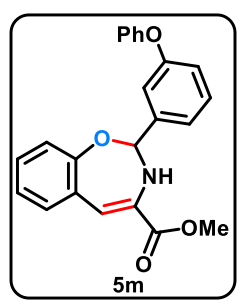

Compound 5m was prepared following the general procedure for synthesis of 1,3Benzoxazepines described above by using $1 \mathrm{a}(0.22 \mathrm{mmol}, 36 \mathrm{mg})$ and purified by silica gel column chromatography $(1 \rightarrow 5 \%$ EtOAc : hexane $)$ to provide pure compound as yellow solid $(60 \mathrm{mg}, 81 \%)$, melting point $=110-112{ }^{\circ} \mathrm{C} .{ }^{1} \mathbf{H}$ NMR $\left(400 \mathrm{MHz}, \mathrm{CDCl}_{3}\right) \delta$ $7.43-7.35(\mathrm{~m}, 3 \mathrm{H}), 7.29-7.26(\mathrm{~m}, 2 \mathrm{H}), 7.23-7.22(\mathrm{~m}, 1 \mathrm{H}), 7.16-7.10(\mathrm{~m}, 2 \mathrm{H}), 7.08$ $-7.02(\mathrm{~m}, 4 \mathrm{H}), 7.01-6.97(\mathrm{~m}, 1 \mathrm{H}), 6.62(\mathrm{~s}, 1 \mathrm{H}), 5.62(\mathrm{~s}, 1 \mathrm{H}), 5.49(\mathrm{~d}, J=4.7 \mathrm{~Hz}, 1 \mathrm{H})$, $3.86(\mathrm{~s}, 3 \mathrm{H}) .{ }^{13} \mathrm{C}$ NMR $\left(101 \mathrm{MHz}, \mathrm{CDCl}_{3}\right) \delta 165.1,158.9,158.0,156.7,140.2,132.6$, 132.6, 130.4, 130.0, 127.8, 125.8, 123.8, 123.2, 121.2, 120.7, 119.4, 119.1, 116.9, 110.7, 88.0, 52.8. HRMS (ESI/TOF-Q) m/z: $[\mathrm{M}+\mathrm{H}]^{+}$Calcd for $\mathrm{C}_{23} \mathrm{H}_{19} \mathrm{NO}_{4} \mathrm{H}^{+} 374.1387$; Found 374.1396.

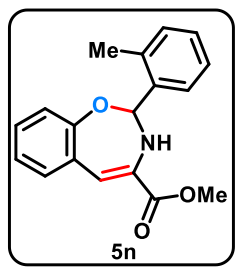

Compound 5n was prepared following the general procedure for synthesis of 1,3Benzoxazepines described above by using $1 \mathrm{a}(0.22 \mathrm{mmol}, 36 \mathrm{mg})$ and purified by silica gel column chromatography ( $\rightarrow 5 \%$ EtOAc : hexane) to provide pure compound as yellow solid $(44 \mathrm{mg}, 75 \%)$, melting point $=88-90{ }^{\circ} \mathrm{C} .{ }^{1} \mathbf{H}$ NMR $\left(400 \mathrm{MHz}, \mathrm{CDCl}_{3}\right) \delta$ $7.63-7.61(\mathrm{~m}, 1 \mathrm{H}), 7.25-7.21(\mathrm{~m}, 3 \mathrm{H}), 7.18-7.14(\mathrm{~m}, 1 \mathrm{H}), 7.06-7.02(\mathrm{~m}, 1 \mathrm{H}), 6.97$ $-6.89(\mathrm{~m}, 2 \mathrm{H}), 6.54(\mathrm{~s}, 1 \mathrm{H}), 5.55(\mathrm{~d}, J=4.4 \mathrm{~Hz}, 1 \mathrm{H}), 5.50(\mathrm{~s}, 1 \mathrm{H}), 3.77(\mathrm{~s}, 3 \mathrm{H}), 2.27(\mathrm{~s}$, $3 \mathrm{H}) .{ }^{13} \mathrm{C}$ NMR $\left(101 \mathrm{MHz}, \mathrm{CDCl}_{3}\right) \delta 165.1,159.2,136.8,135.3,132.8,132.5,130.8$, $129.0,127.8,126.7,126.5,125.9,123.1,120.7,110.4,86.1,52.8,19.2$. HRMS (ESI/TOF-Q) m/z: [M+H] ${ }^{+}$Calcd for $\mathrm{C}_{18} \mathrm{H}_{17} \mathrm{NO}_{3} \mathrm{H}^{+} 296.1281$; Found 296.1287.

Compound 5o was prepared following the general procedure for synthesis of 1,3Benzoxazepines described above by using $1 \mathrm{a}(0.22 \mathrm{mmol}, 36 \mathrm{mg})$ and purified by silica gel column chromatography ( $\rightarrow 5 \%$ EtOAc : hexane) to provide pure compound as yellow solid (42 mg, 70\%), melting point $=102-104{ }^{\circ} \mathrm{C} .{ }^{1} \mathbf{H}$ NMR $\left(400 \mathrm{MHz}, \mathrm{CDCl}_{3}\right) \delta$ $7.74-7.70(\mathrm{~m}, 1 \mathrm{H}), 7.45-7.39(\mathrm{~m}, 1 \mathrm{H}), 7.31-7.27(\mathrm{~m}, 2 \mathrm{H}), 7.14-7.10(\mathrm{~m}, 2 \mathrm{H}), 7.05$ $-7.02(\mathrm{~m}, 1 \mathrm{H}), 6.98-6.96(\mathrm{~m}, 1 \mathrm{H}), 6.65(\mathrm{~s}, 1 \mathrm{H}), 5.80(\mathrm{~d}, J=4.4 \mathrm{~Hz}, 1 \mathrm{H}), 5.63(\mathrm{~s}, 1 \mathrm{H})$, $3.86(\mathrm{~s}, 3 \mathrm{H}) .{ }^{13} \mathrm{C}$ NMR $\left(101 \mathrm{MHz}, \mathrm{CDCl}_{3}\right) \delta 165.1,159.3(\mathrm{~d}, J=249.1 \mathrm{~Hz}), 158.9$, $132.8,132.6,131.0(\mathrm{~d}, J=8.3 \mathrm{~Hz}), 128.2(\mathrm{~d}, J=3.2 \mathrm{~Hz}), 127.9,125.8,125.0(\mathrm{~d}, J=3.6$ $\mathrm{Hz}), 123.3,120.7,115.8(\mathrm{~d}, J=21.4 \mathrm{~Hz}), 110.8,82.5,82.4,52.9 .{ }^{19} \mathbf{F}$ NMR $(471 \mathrm{MHz}$, $\left.\mathrm{CDCl}_{3}\right) \delta-118.98$ (s). HRMS (ESI/TOF-Q) m/z: $[\mathrm{M}+\mathrm{H}]^{+}$Calcd for $\mathrm{C}_{17} \mathrm{H}_{14} \mathrm{FNO}_{3} \mathrm{H}^{+}$ 300.1030 ; Found 300.1044.

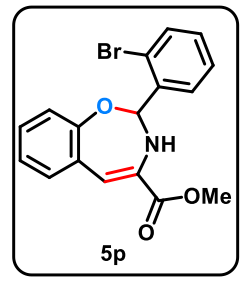

Compound 5p was prepared following the general procedure for synthesis of 1,3Benzoxazepines described above by using $1 \mathbf{a}(0.22 \mathrm{mmol}, 36 \mathrm{mg})$ and purified by silica gel column chromatography ( $\rightarrow 5 \%$ EtOAc : hexane) to provide pure compound as yellow solid $(53 \mathrm{mg}, 74 \%)$, melting point $=130-132{ }^{\circ} \mathrm{C} .{ }^{1} \mathbf{H}$ NMR $\left(400 \mathrm{MHz}, \mathrm{CDCl}_{3}\right) \delta$ $7.84-7.82(\mathrm{~m}, 1 \mathrm{H}), 7.60(\mathrm{~d}, J=8.1 \mathrm{~Hz}, 1 \mathrm{H}), 7.47(\mathrm{t}, J=7.6 \mathrm{~Hz}, 1 \mathrm{H}), 7.31-7.27(\mathrm{~m}$, 2H), $7.15-7.11(\mathrm{~m}, 1 \mathrm{H}), 7.06-6.98(\mathrm{~m}, 2 \mathrm{H}), 6.65(\mathrm{~s}, 1 \mathrm{H}), 5.79(\mathrm{~d}, J=3.9 \mathrm{~Hz}, 1 \mathrm{H})$, $5.55(\mathrm{~s}, 1 \mathrm{H}), 3.87(\mathrm{~s}, 3 \mathrm{H}) .{ }^{13} \mathrm{C}$ NMR $\left(101 \mathrm{MHz}, \mathrm{CDCl}_{3}\right) \delta 165.0,158.7,137.9,132.9$, $132.8,132.7,130.7,128.5,128.3,127.9,125.7,123.3,122.3,120.7,110.7,87.2,52.9$. HRMS (ESI/TOF-Q) m/z: $[\mathrm{M}+\mathrm{Na}]^{+}$Calcd for $\mathrm{C}_{17} \mathrm{H}_{14} \mathrm{BrNO}_{3} \mathrm{Na}^{+} 382.0049$; Found 382.0053 . 

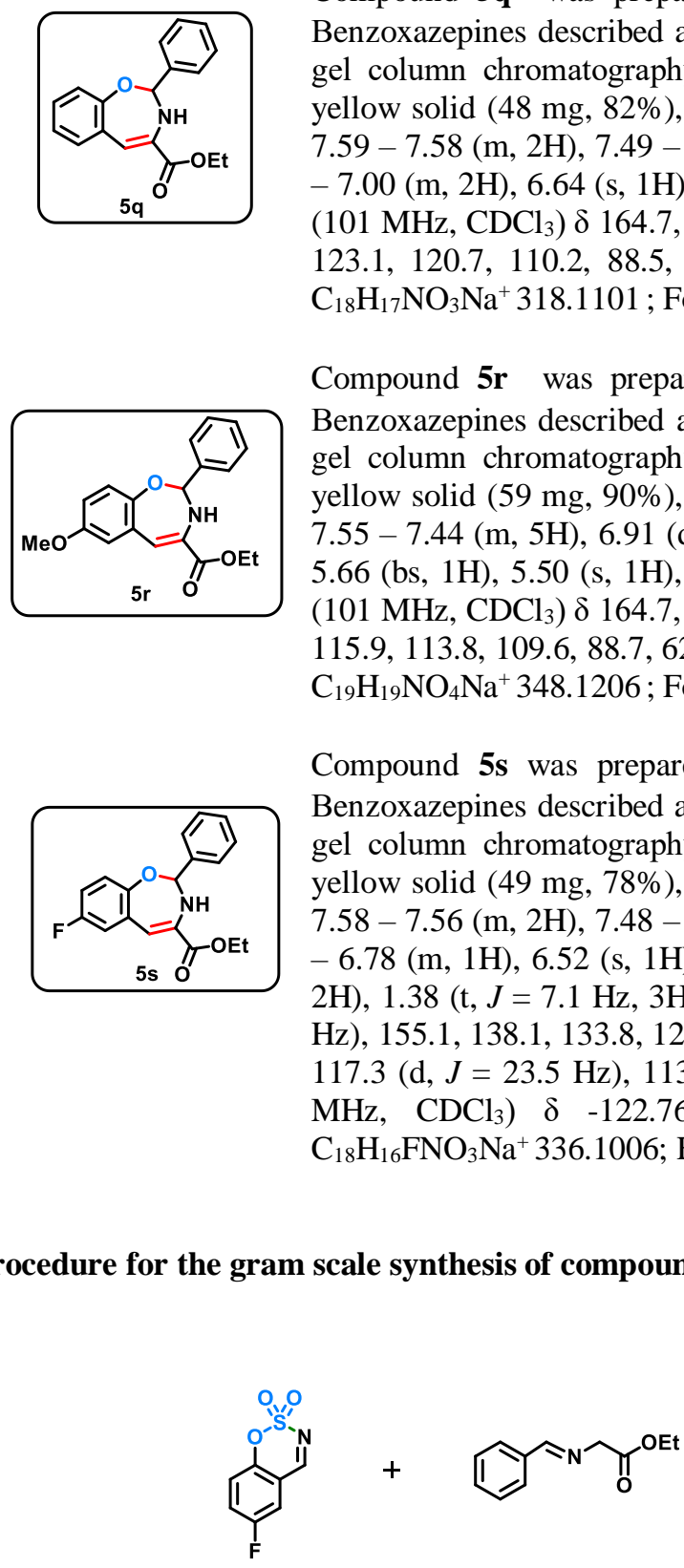

\section{Procedure for the gram scale synthesis of compound 5s:}

Compound 5q was prepared following the general procedure for synthesis of 1,3Benzoxazepines described above by using $1 \mathbf{a}(0.22 \mathrm{mmol}, 36 \mathrm{mg})$ and purified by silica gel column chromatography ( $\rightarrow 5 \%$ EtOAc : hexane) to provide pure compound as yellow solid $(48 \mathrm{mg}, 82 \%)$, melting point $=118-120{ }^{\circ} \mathrm{C} .{ }^{1} \mathbf{H}$ NMR $\left(400 \mathrm{MHz}, \mathrm{CDCl}_{3}\right) \delta$ $7.59-7.58(\mathrm{~m}, 2 \mathrm{H}), 7.49-7.42(\mathrm{~m}, 3 \mathrm{H}), 7.33-7.31(\mathrm{~m}, 1 \mathrm{H}), 7.15-7.11(\mathrm{~m}, 1 \mathrm{H}), 7.06$ $-7.00(\mathrm{~m}, 2 \mathrm{H}), 6.64(\mathrm{~s}, 1 \mathrm{H}), 4.33(\mathrm{q}, J=6.9 \mathrm{~Hz}, 2 \mathrm{H}), 1.38(\mathrm{t}, J=6.7 \mathrm{~Hz}, 3 \mathrm{H}) .{ }^{13} \mathbf{C}$ NMR $\left(101 \mathrm{MHz}, \mathrm{CDCl}_{3}\right) \delta 164.7,159.1,138.5,133.1,132.5,129.2,129.0,127.7,126.7,126.0$, 123.1, 120.7, 110.2, 88.5, 61.9, 14.4. HRMS (ESI/TOF-Q) m/z: $[\mathrm{M}+\mathrm{Na}]^{+}$Calcd for $\mathrm{C}_{18} \mathrm{H}_{17} \mathrm{NO}_{3} \mathrm{Na}^{+} 318.1101$; Found 318.1107.

Compound $\mathbf{5 r}$ was prepared following the general procedure for synthesis of 1,3Benzoxazepines described above by using $1 \mathbf{d}(0.22 \mathrm{mmol}, 43 \mathrm{mg})$ and purified by silica gel column chromatography $(1 \rightarrow 5 \%$ EtOAc : hexane $)$ to provide pure compound as yellow solid (59 mg, 90\%), melting point $=105-107^{\circ} \mathrm{C} .{ }^{1} \mathbf{H}$ NMR $\left(400 \mathrm{MHz}, \mathrm{CDCl}_{3}\right) \delta$ $7.55-7.44(\mathrm{~m}, 5 \mathrm{H}), 6.91(\mathrm{~d}, J=7.8 \mathrm{~Hz}, 1 \mathrm{H}), 6.80(\mathrm{bs}, 1 \mathrm{H}), 6.70(\mathrm{bs}, 1 \mathrm{H}), 6.56(\mathrm{~s}, 1 \mathrm{H})$, $5.66(\mathrm{bs}, 1 \mathrm{H}), 5.50(\mathrm{~s}, 1 \mathrm{H}), 4.33-4.31(\mathrm{~m}, 2 \mathrm{H}), 3.80(\mathrm{~s}, 3 \mathrm{H}), 1.37(\mathrm{bs}, 3 \mathrm{H}) .{ }^{13} \mathbf{C}$ NMR $\left(101 \mathrm{MHz}, \mathrm{CDCl}_{3}\right) \delta 164.7,155.3,153.3,138.6,133.3,129.2,129.0,126.9,126.7,121.4$, 115.9, 113.8, 109.6, 88.7, 62.0, 55.8, 14.4. HRMS (ESI/TOF-Q) m/z: $[\mathrm{M}+\mathrm{Na}]^{+}$Calcd for $\mathrm{C}_{19} \mathrm{H}_{19} \mathrm{NO}_{4} \mathrm{Na}^{+} 348.1206$; Found 348.1215

Compound 5s was prepared following the general procedure for synthesis of 1,3Benzoxazepines described above by using $\mathbf{1 h}(0.22 \mathrm{mmol}, 40 \mathrm{mg})$ and purified by silica gel column chromatography $(1 \rightarrow 5 \%$ EtOAc : hexane) to provide pure compound as yellow solid (49 mg, 78\%), melting point $=120-122{ }^{\circ} \mathrm{C} .{ }^{1} \mathbf{H}$ NMR $\left(400 \mathrm{MHz}, \mathrm{CDCl}_{3}\right) \delta$ $7.58-7.56(\mathrm{~m}, 2 \mathrm{H}), 7.48-7.42(\mathrm{~m}, 3 \mathrm{H}), 7.00-6.97(\mathrm{~m}, 1 \mathrm{H}), 6.94-6.91(\mathrm{~m}, 1 \mathrm{H}), 6.82$ $-6.78(\mathrm{~m}, 1 \mathrm{H}), 6.52(\mathrm{~s}, 1 \mathrm{H}), 5.77(\mathrm{~s}, 1 \mathrm{H}), 5.50(\mathrm{~d}, J=4.6 \mathrm{~Hz}, 1 \mathrm{H}), 4.33(\mathrm{q}, J=7.1 \mathrm{~Hz}$, $2 \mathrm{H}), 1.38(\mathrm{t}, J=7.1 \mathrm{~Hz}, 3 \mathrm{H}) .{ }^{13} \mathbf{C}$ NMR $\left(101 \mathrm{MHz}, \mathrm{CDCl}_{3}\right) \delta 164.4,158.5(\mathrm{~d}, J=239.8$ $\mathrm{Hz}), 155.1,138.1,133.8,129.3,128.9,127.7(\mathrm{~d}, J=8.3 \mathrm{~Hz}), 126.6,121.6(\mathrm{~d}, J=8.7 \mathrm{~Hz})$, $117.3(\mathrm{~d}, J=23.5 \mathrm{~Hz}), 113.9(\mathrm{~d}, J=23.4 \mathrm{~Hz}), 108.3,88.6,62.0,14.3 .{ }^{19} \mathbf{F}$ NMR $(471$ $\left.\mathrm{MHz}, \quad \mathrm{CDCl}_{3}\right) \delta-122.76$ (s). HRMS (ESI/TOF-Q) $\mathrm{m} / \mathrm{z}: \quad[\mathrm{M}+\mathrm{Na}]^{+}$Calcd for $\mathrm{C}_{18} \mathrm{H}_{16} \mathrm{FNO}_{3} \mathrm{Na}^{+}$336.1006; Found 336.1013.
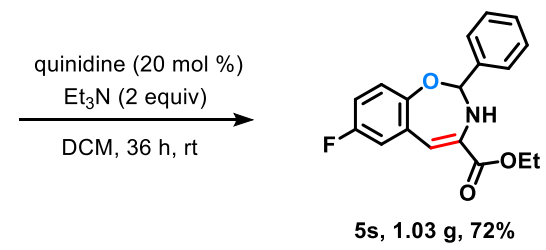

In an oven dried $100 \mathrm{~mL}$ round bottom flask equipped with a magnetic stir bar, fluorine substituted sulfamatederived imine ( $0.915 \mathrm{~g}, 1.0$ equiv), ethyl glycine aldimino ester $(0.962 \mathrm{~g}, 1.1$ equiv), and quinidine ( $0.296 \mathrm{~g}, 0.2$ equiv) were taken. Then, DCM $(15 \mathrm{~mL})$ followed $\mathrm{Et}_{3} \mathrm{~N}(1.28 \mathrm{~mL}, 2.0$ equiv) were added and the reaction mixture was allowed to stir at room temperature. After $36 \mathrm{~h}$, the reaction mixture was monitored by thin layer chromatography and concentrated under reduced pressure. The crude product was purified by silica gel column chromatography $(1 \rightarrow 5 \%$ EtOAc $:$ hexane) to provide pure product $5 \mathrm{~s}(1.03 \mathrm{~g}, 72 \%)$. 


\section{Procedure for the synthesis of ortho-tyrosine amino acid derivatives 6:}

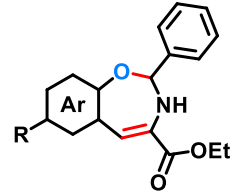

5

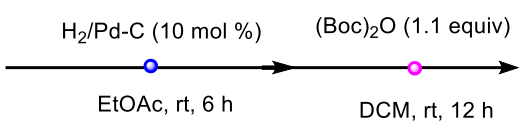

DCM, rt, $12 \mathrm{~h}$

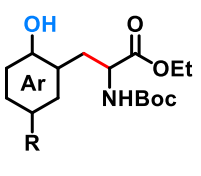

6

Compound $5(0.2 \mathrm{mmol})$ was added into flame-dried tube equipped with a magnetic stir bar, $\mathrm{Pd} / \mathrm{C}(10 \mathrm{wt} \%, 10 \mathrm{~mol}$ $\%)$ and ethyl acetate $(2.0 \mathrm{~mL})$ were added under inert atmosphere. The reaction mixture was degassed followed by hydrogen gas was charged and stirred at room temperature for $6 \mathrm{~h}$. Then, the reaction mixture was filtered through a pad of Celite and concentrated under reduced pressure. This crude product was again taken into flame-dried reaction tube equipped with a magnetic stir bar. DCM $(2 \mathrm{~mL})$ and $(\mathrm{Boc})_{2} \mathrm{O}(1.1$ equiv) were added and the mixture was stirred at room temperature for $12 \mathrm{~h}$. The reaction mixture was concentrated under reduced pressure and the crude product was purified by silica gel column chromatography $(10 \rightarrow 15 \%$ EtOAc $:$ hexane) to obtain the pure product 6 .
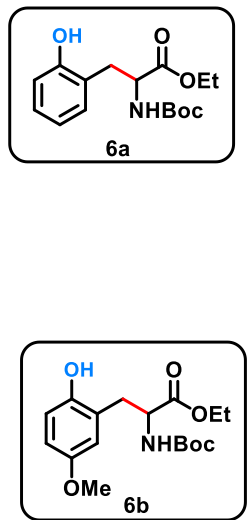

Compound 6a was prepared following the general procedure for synthesis of ortho-tyrosine amino acid derivative described above by using $\mathbf{5 q}(0.2 \mathrm{mmol}, 58 \mathrm{mg})$ and purified by silica gel column chromatography $(10 \rightarrow 15 \%$ EtOAc : hexane) to provide pure compound as white solid $(54 \mathrm{mg}, 88 \%)$, melting point $=142-144{ }^{\circ} \mathrm{C} .{ }^{1} \mathbf{H}$ NMR $\left(400 \mathrm{MHz}, \mathrm{CDCl}_{3}\right) \delta 7.85(\mathrm{bs}$, $1 \mathrm{H}), 7.10-7.03(\mathrm{~m}, 2 \mathrm{H}), 6.84-6.78(\mathrm{~m}, 2 \mathrm{H}), 5.63(\mathrm{~s}, 1 \mathrm{H}), 4.51(\mathrm{~s}, 1 \mathrm{H}), 4.38-3.95(\mathrm{~m}, 2 \mathrm{H})$, $3.28-2.83(\mathrm{~m}, 2 \mathrm{H}), 1.42(\mathrm{~s}, 9 \mathrm{H}), 1.23(\mathrm{bs}, 3 \mathrm{H}) .{ }^{13} \mathrm{C}$ NMR $\left(101 \mathrm{MHz}, \mathrm{CDCl}_{3}\right) \delta 172.8,156.4$, 154.9, 131.4, 128.7, 123.0, 120.0, 115.7, 80.9, 61.7, 54.2, 34.3, 28.4, 14.2. HRMS (ESI/TOFQ) $\mathrm{m} / \mathrm{z}$ : $[\mathrm{M}+\mathrm{Na}]^{+}$Calcd for $\mathrm{C}_{16} \mathrm{H}_{23} \mathrm{NO}_{5} \mathrm{Na}^{+} 332.1468$; Found 332.1474.

Compound $\mathbf{6 b}$ was prepared following the general procedure for synthesis of ortho-tyrosine amino acid derivative described above by using $\mathbf{5 r}(0.2 \mathrm{mmol}, 64 \mathrm{mg})$ and purified by silica gel column chromatography $(10 \rightarrow 15 \%$ EtOAc : hexane) to provide pure compound as white solid $(62 \mathrm{mg}, 92 \%)$, melting point $=152-154{ }^{\circ} \mathrm{C} .{ }^{1} \mathbf{H}$ NMR $\left(400 \mathrm{MHz}, \mathrm{CDCl}_{3}\right) \delta 7.23(\mathrm{~s}, 1 \mathrm{H})$, $6.76-6.74(\mathrm{~m}, 1 \mathrm{H}), 6.65-6.61(\mathrm{~m}, 2 \mathrm{H}), 5.61(\mathrm{bs}, 1 \mathrm{H}), 4.49(\mathrm{~s}, 1 \mathrm{H}), 4.19-4.18(\mathrm{~m}, 2 \mathrm{H})$, $3.72(\mathrm{~s}, 3 \mathrm{H}), 3.10-3.02(\mathrm{~m}, 2 \mathrm{H}), 1.42(\mathrm{~s}, 9 \mathrm{H}), 1.25$ (bs, 3H). ${ }^{13} \mathbf{C}$ NMR $\left(101 \mathrm{MHz}, \mathrm{CDCl}_{3}\right) \delta$ 172.7, 157.5, 153.1, 149.0, 123.7, 116.9, 113.7 (2xC), 80.6, 61.7, 55.9, 54.3, 34.3, 28.4, 14.3. HRMS (ESI/TOF-Q) m/z: [M+Na] ${ }^{+}$Calcd for $\mathrm{C}_{17} \mathrm{H}_{25} \mathrm{NO}_{6} \mathrm{Na}^{+} 362.1574$; Found 362.1583 .

Compound $\mathbf{6 c}$ was prepared following the general procedure for synthesis of ortho-tyrosine

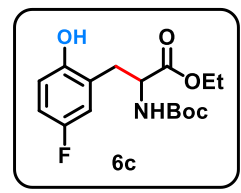
amino acid derivative described above by using $5 \mathrm{~s}(0.2 \mathrm{mmol}, 63 \mathrm{mg})$ and purified by silica gel column chromatography $(10 \rightarrow 15 \%$ EtOAc : hexane) to provide pure compound as white solid $(56 \mathrm{mg}, 85 \%)$, melting point $=128-130{ }^{\circ} \mathrm{C} .{ }^{1} \mathbf{H}$ NMR $\left(400 \mathrm{MHz}, \mathrm{CDCl}_{3}\right) \delta 7.83(\mathrm{bs}$, $1 \mathrm{H}), 6.76-6.75(\mathrm{~m}, 3 \mathrm{H}), 5.60(\mathrm{bs}, 1 \mathrm{H}), 4.47$ (bs, $1 \mathrm{H}), 4.20-4.19(\mathrm{~m}, 2 \mathrm{H}), 3.08-2.97(\mathrm{~m}$, 2H), $1.42(\mathrm{~s}, 9 \mathrm{H}), 1.25(\mathrm{bs}, 3 \mathrm{H}) .{ }^{13} \mathbf{C}$ NMR $\left(101 \mathrm{MHz}, \mathrm{CDCl}_{3}\right) \delta 172.2,157.5,155.7(\mathrm{~d}, J=$ $87.1 \mathrm{~Hz}), 151.3,124.2,117.4(\mathrm{~d}, J=6.1 \mathrm{~Hz}), 116.8(\mathrm{~d}, J=8.2 \mathrm{~Hz}), 114.8(\mathrm{~d}, J=22.6 \mathrm{~Hz})$, 80.9, 61.9, 53.9, 34.6, 28.4, 14.2. HRMS (ESI/TOF-Q) $\mathrm{m} / \mathrm{z}:[\mathrm{M}+\mathrm{Na}]^{+}$Calcd for $\mathrm{C}_{16} \mathrm{H}_{22} \mathrm{FNO}_{5} \mathrm{Na}^{+} 350.1374$; Found 350.1384 . 


\section{Crystallographic experimental section:}

Table1. Crystal data and structure refinement for 5d (CCDC number: 2071537), Ellipsoid Probability $50)$ :

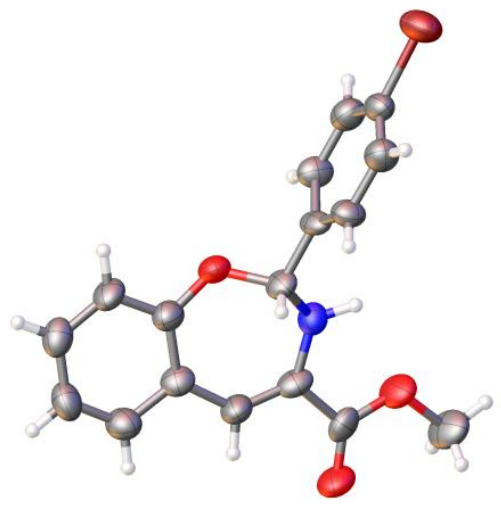

Identification code

Empirical formula

Formula weight

Temperature

Wavelength

Crystal system

Space group

Unit cell dimensions

Volume

Z

Density (calculated)

Absorption coefficient

$\mathrm{F}(000)$

Crystal size

Theta range for data collection

Index ranges

Reflections collected

Independent reflections

Completeness to theta $=24.998^{\circ}$

Absorption correction

Max. and min. transmission

Refinement method 5d

C17 H14 Br N O3

360.20

296(2) K

$0.71073 \AA$

Monoclinic

P 21/c

$\mathrm{a}=7.660(3) \AA \quad \alpha=90^{\circ}$.

$\mathrm{b}=9.587(3) \AA \quad \beta=90.738(11)^{\circ}$.

$\mathrm{c}=20.846(7) \AA \quad \gamma=90^{\circ}$.

$1530.7(9) \AA^{3}$

4

$1.563 \mathrm{Mg} / \mathrm{m}^{3}$

$2.697 \mathrm{~mm}^{-1}$

728

$0.300 \times 0.250 \times 0.200 \mathrm{~mm}^{3}$

0.977 to $24.998^{\circ}$.

$-9<=\mathrm{h}<=9,-11<=\mathrm{k}<=11,-24<=\mathrm{l}<=24$

14149

$2697[\mathrm{R}(\mathrm{int})=0.0938]$

$100.0 \%$

Semi-empirical from equivalents

0.7454 and 0.5786

Full-matrix least-squares on $\mathrm{F}^{2}$ 
Data / restraints / parameters

Goodness-of-fit on $\mathrm{F}^{2}$

Final $\mathrm{R}$ indices [I $>2 \operatorname{sigma}(\mathrm{I})]$

$\mathrm{R}$ indices (all data)

Extinction coefficient

Largest diff. peak and hole 0.708 and -0.975 e. $\AA^{-3}$
2697 / 0 / 204

1.079

$\mathrm{R} 1=0.0955, \mathrm{wR} 2=0.2631$

$\mathrm{R} 1=0.1399, \mathrm{wR} 2=0.3048$

n/a

Note: Compound 5d was dissolved in mixture of hexane: ethyl acetate (10:1) and allowed to evaporate slowly at room temperature for obtain crystal. 
NMR Spectra 


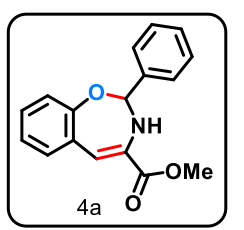

$400 \mathrm{MHz}, \mathrm{CDCl}_{3}$
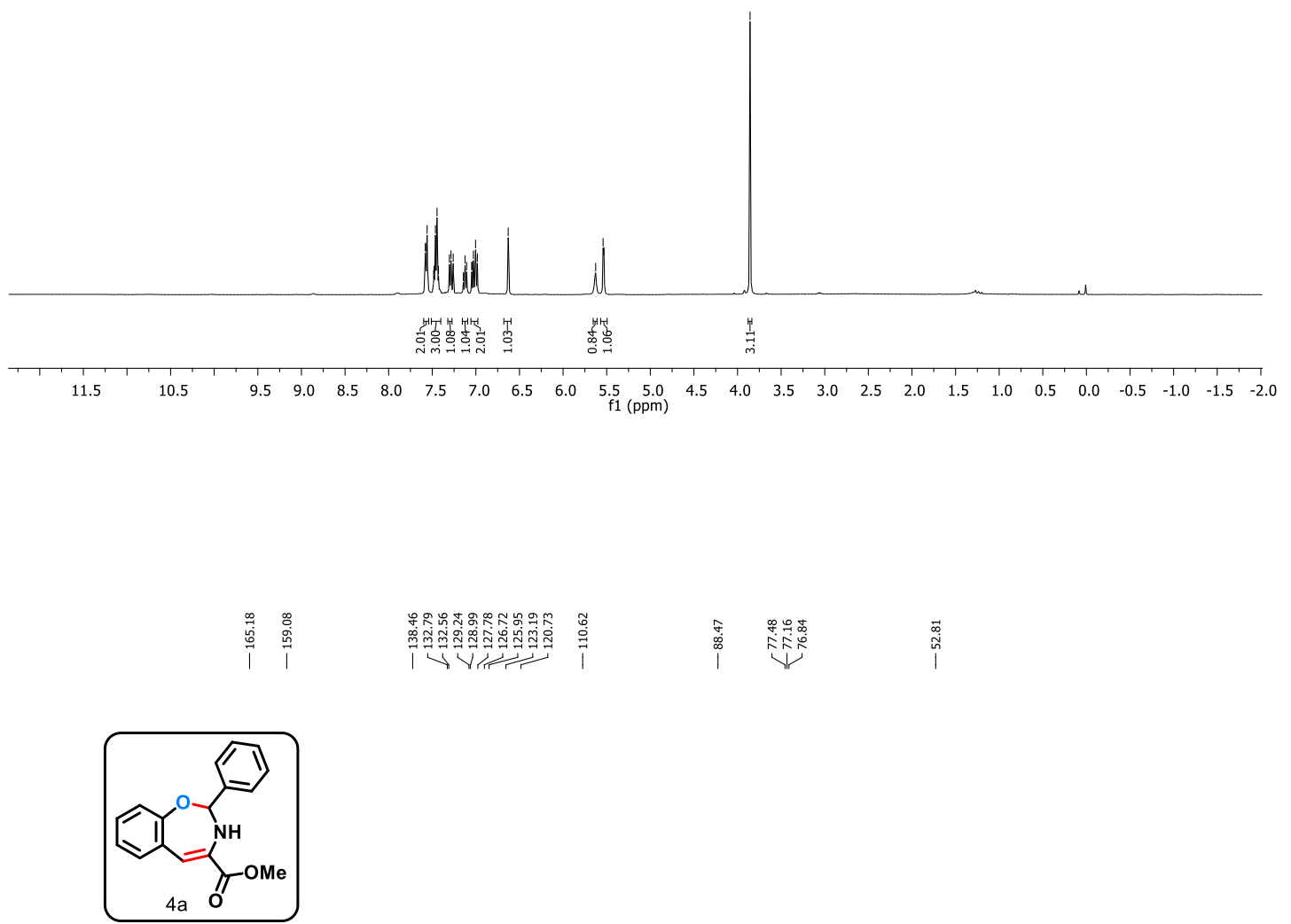

$101 \mathrm{MHz}, \mathrm{CDCl}_{3}$
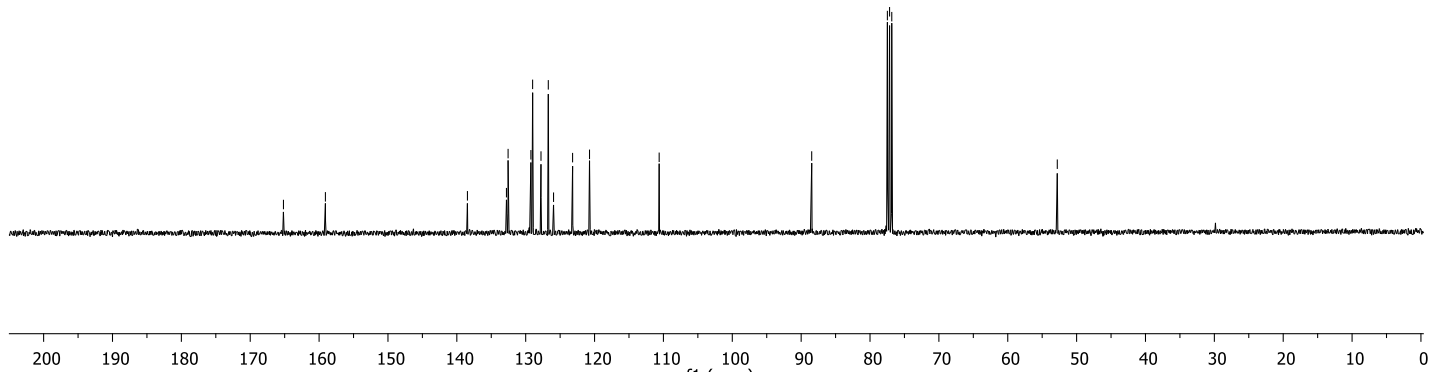


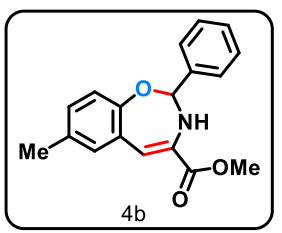

$400 \mathrm{MHz}, \mathrm{CDCl}_{3}$

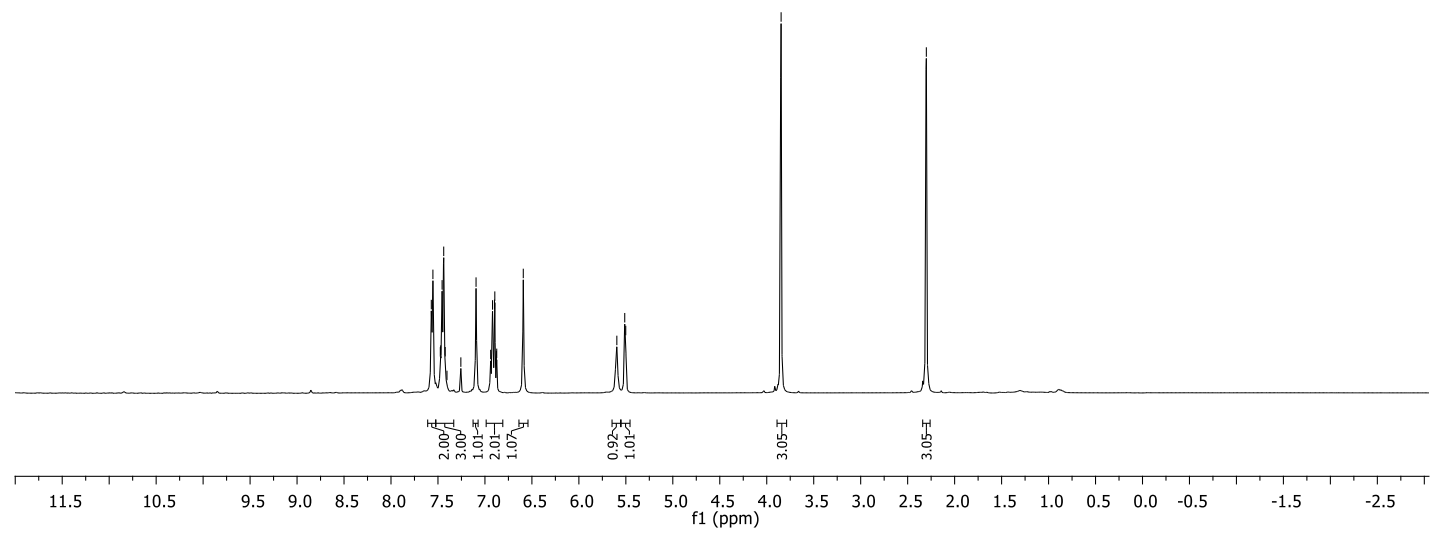

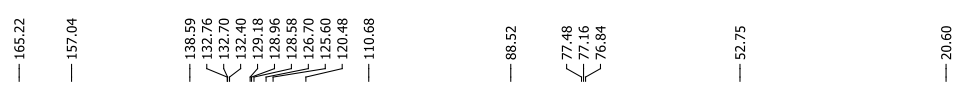

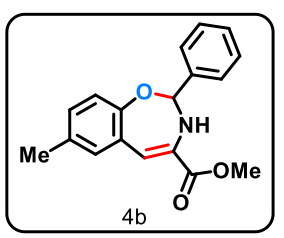

$101 \mathrm{MHz}, \mathrm{CDCl}_{3}$

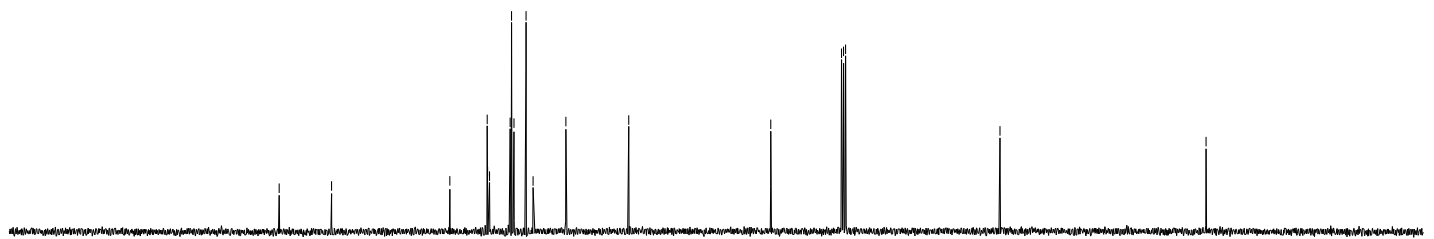

$\begin{array}{lllllllllll}200 & 190 & 180 & 170 & 160 & 150 & 140 & 130 & 120 & 110 & 100 \\ \mathrm{f} 1(\mathrm{ppm}) & 90\end{array}$ 

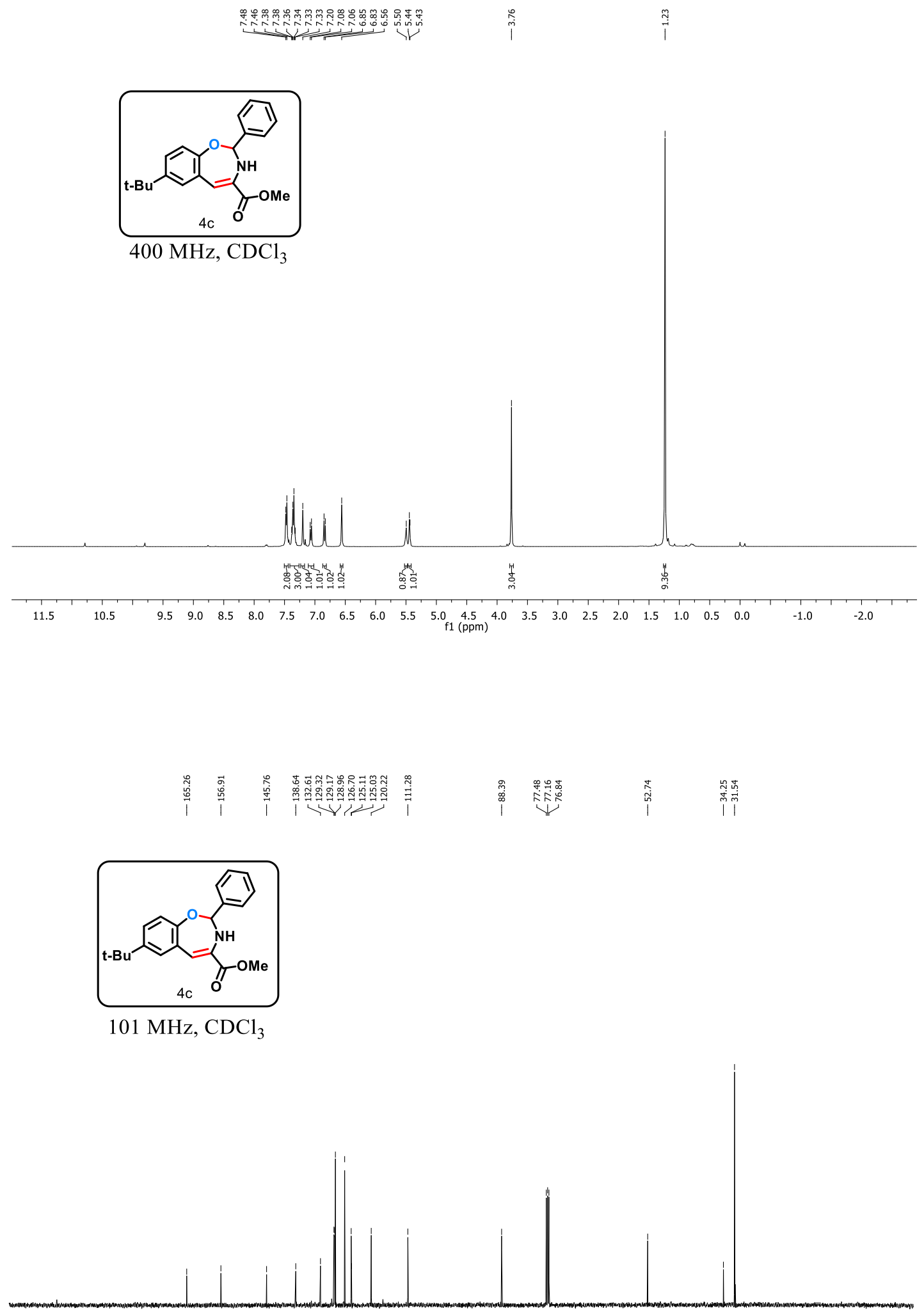

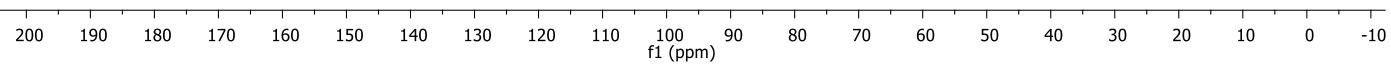




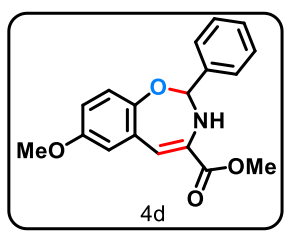

$400 \mathrm{MHz}, \mathrm{CDCl}_{3}$

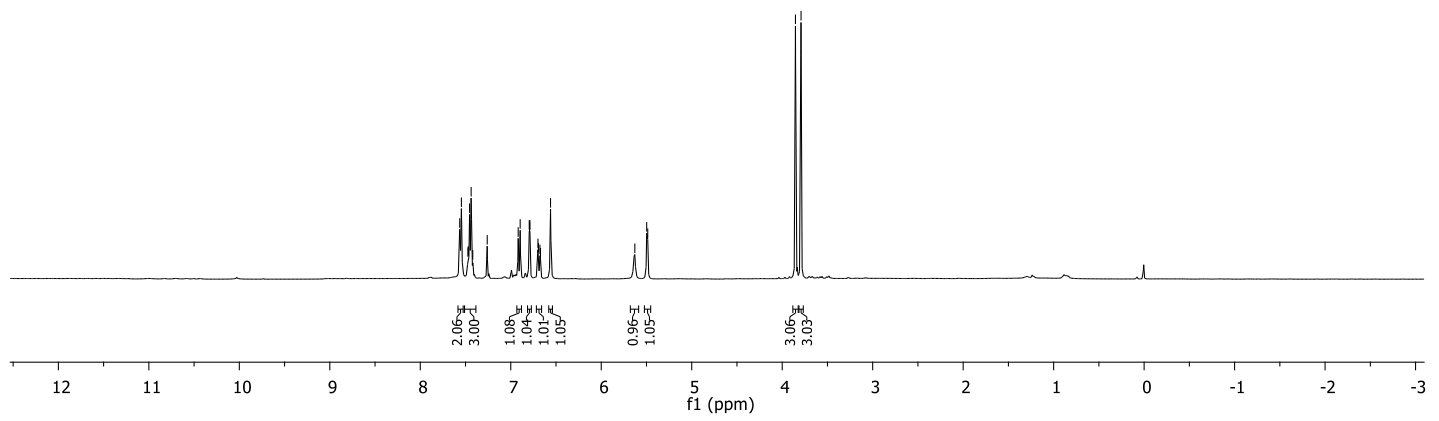

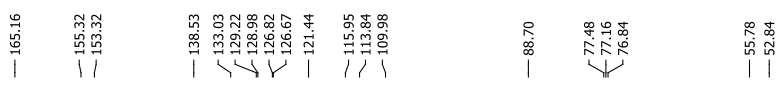

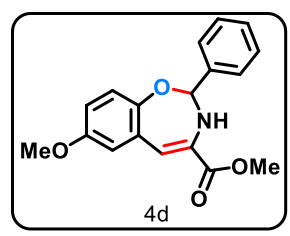

$101 \mathrm{MHz}, \mathrm{CDCl}_{3}$
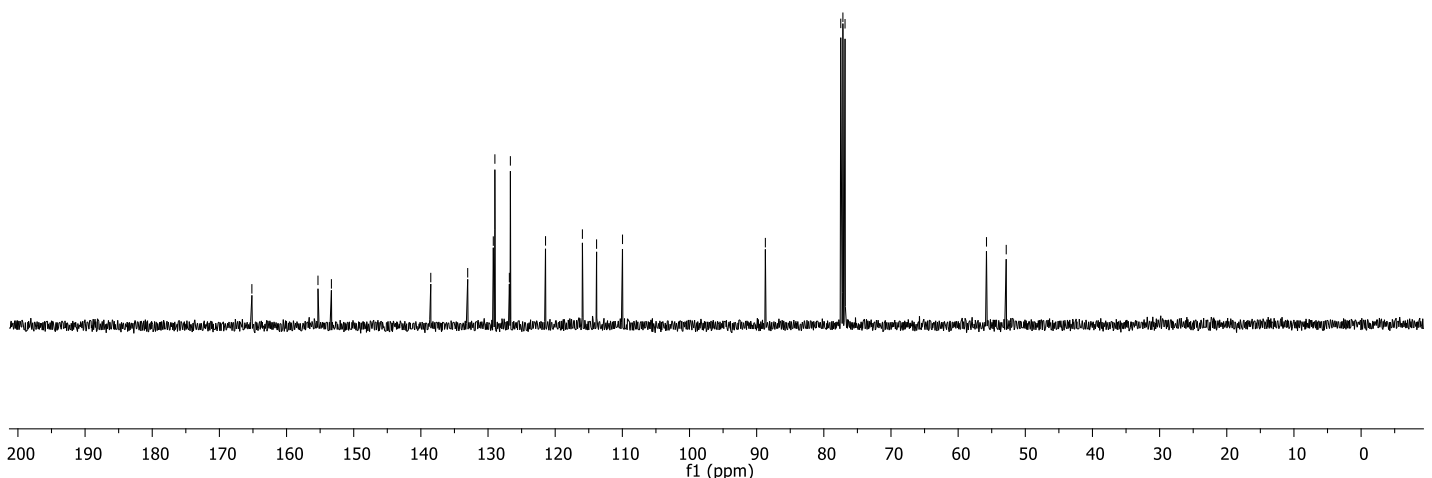


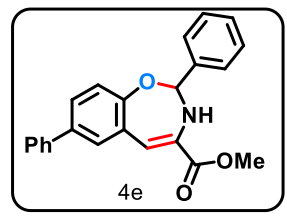

$400 \mathrm{MHz}, \mathrm{CDCl}_{3}$
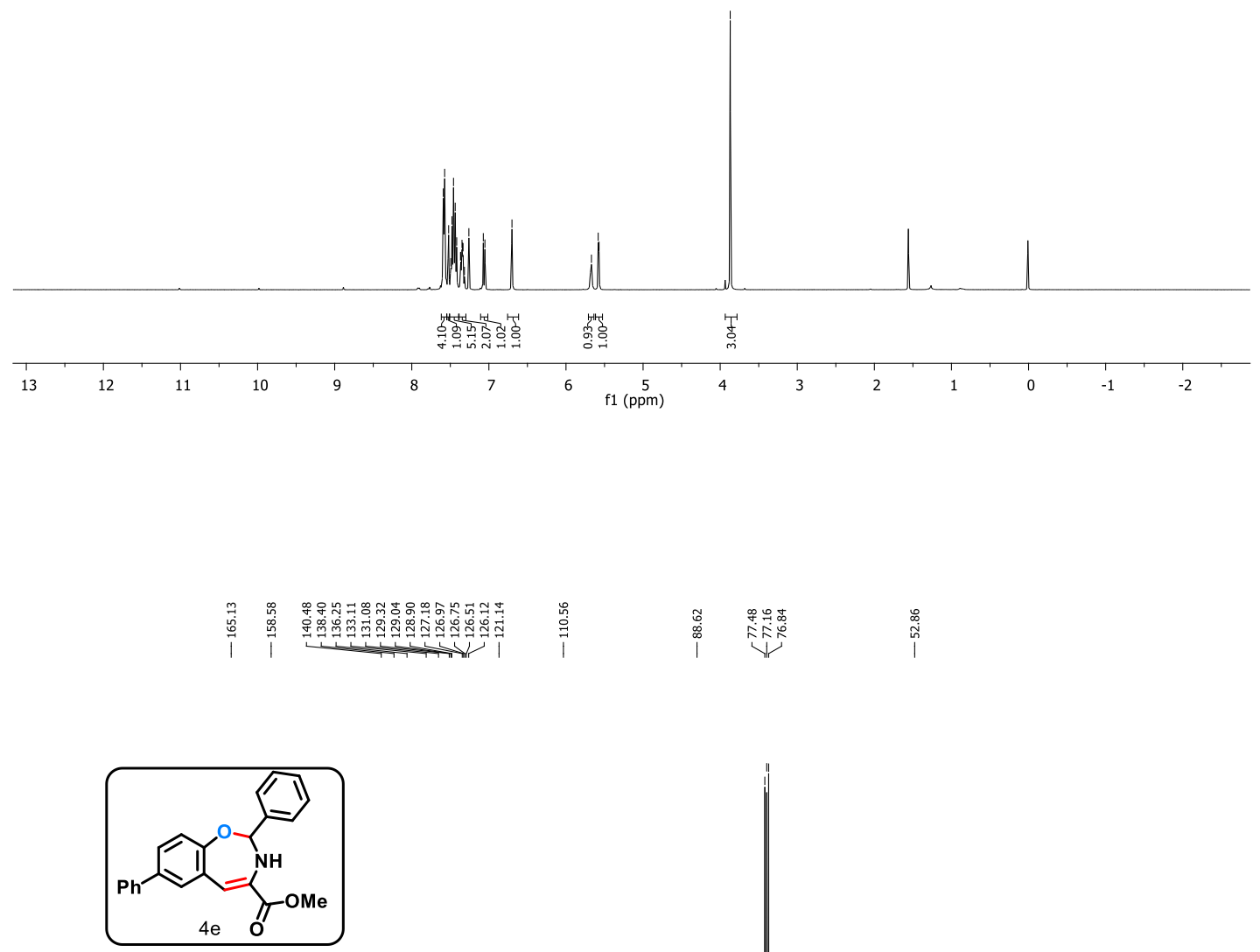

$101 \mathrm{MHz}, \mathrm{CDCl}_{3}$
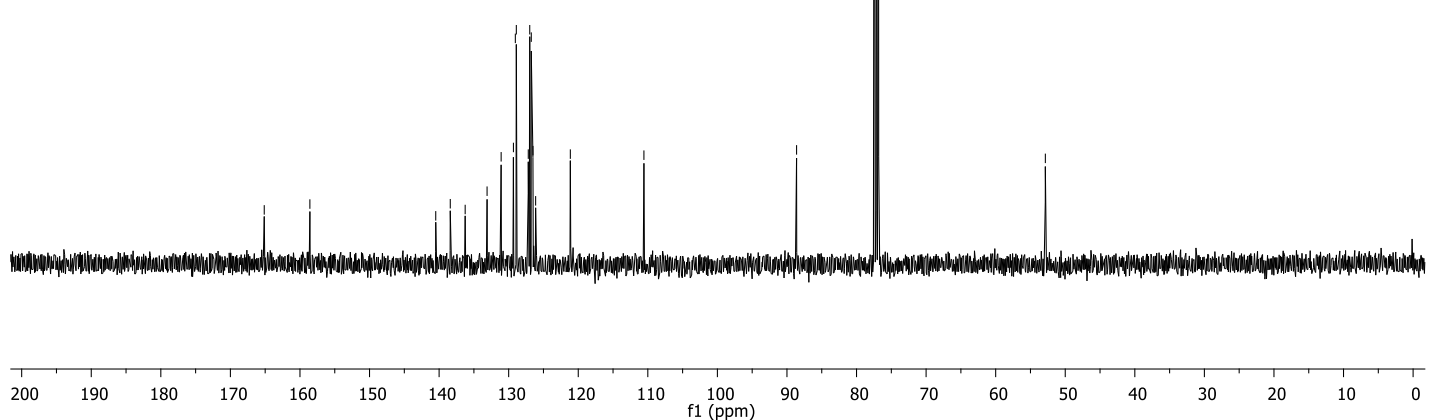


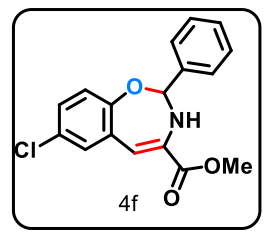

$400 \mathrm{MHz}, \mathrm{CDCl}_{3}$

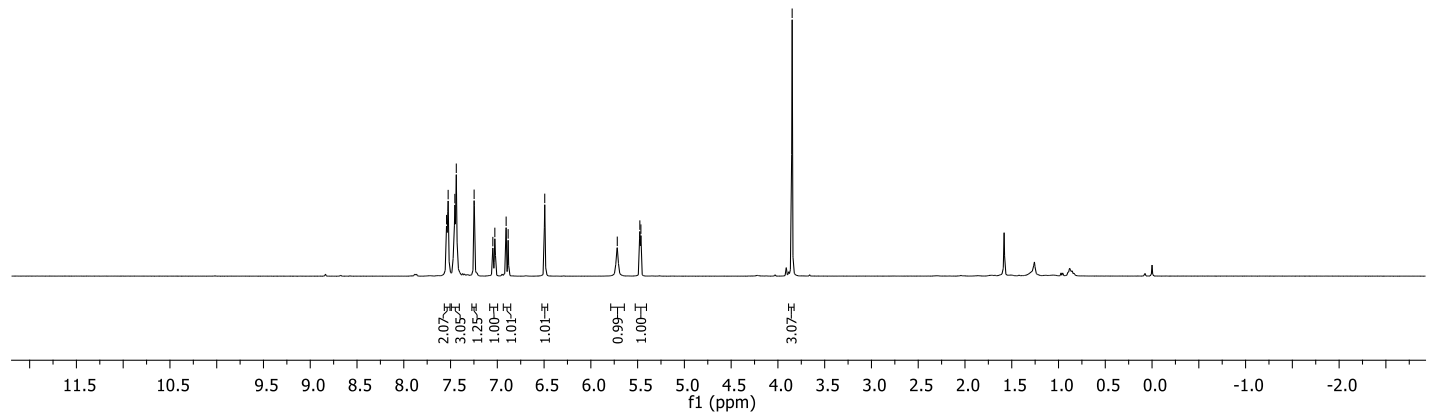

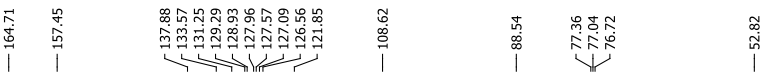

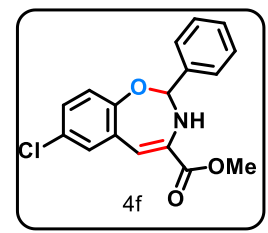

$101 \mathrm{MHz}, \mathrm{CDCl}_{3}$

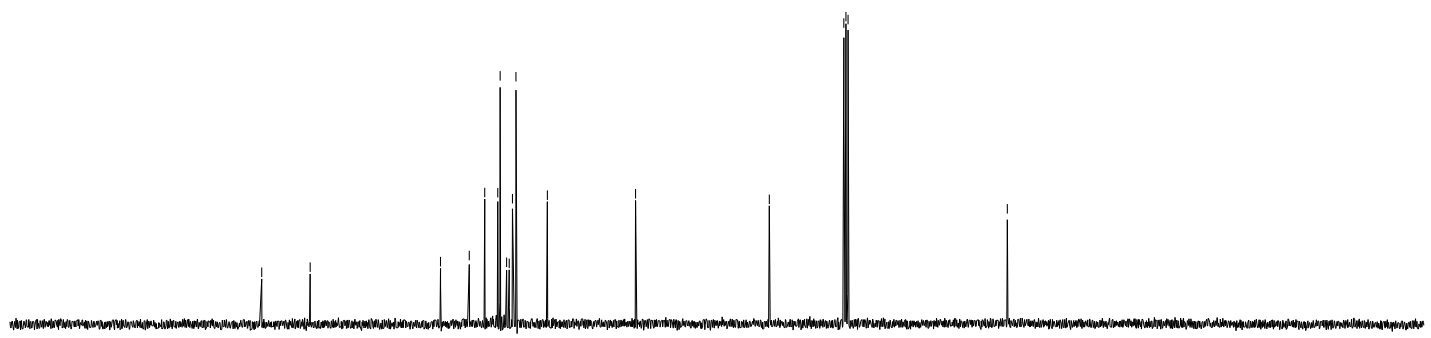

200
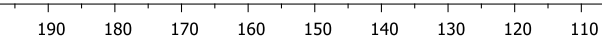

${ }_{\mathrm{f} 1(\mathrm{ppm})}^{100}$ 


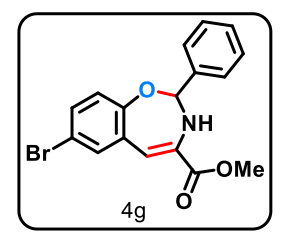

$400 \mathrm{MHz}, \mathrm{CDCl}_{3}$

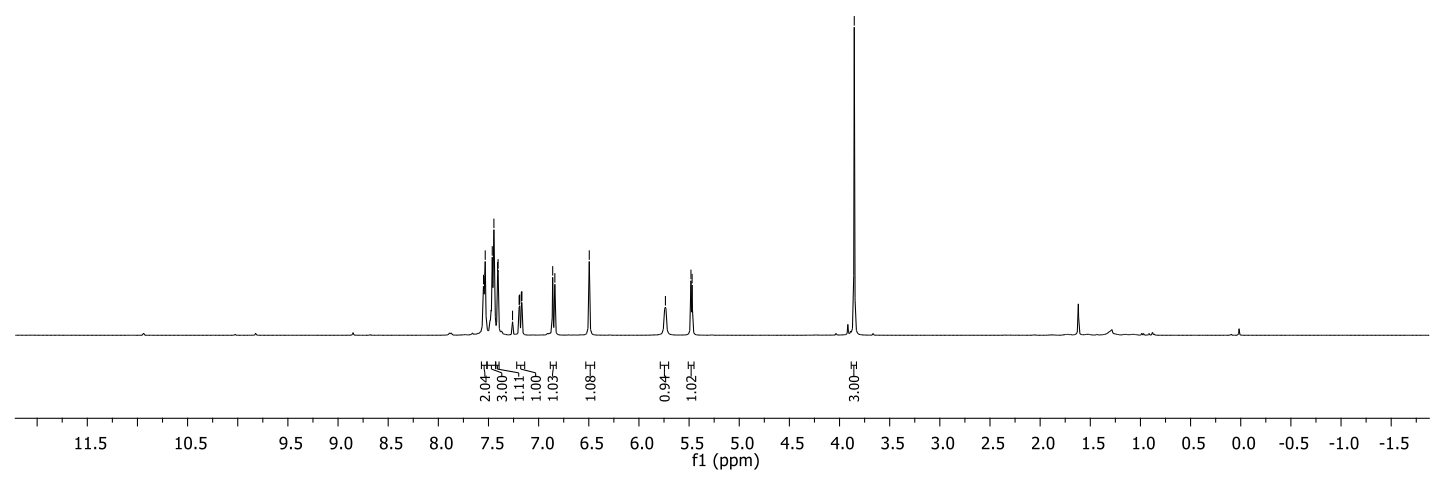

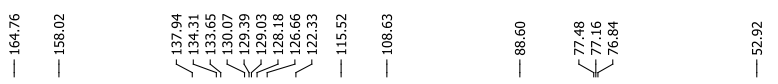

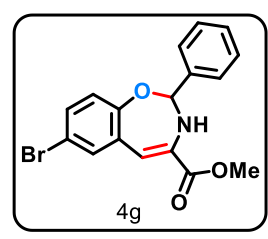

$101 \mathrm{MHz}, \mathrm{CDCl}_{3}$
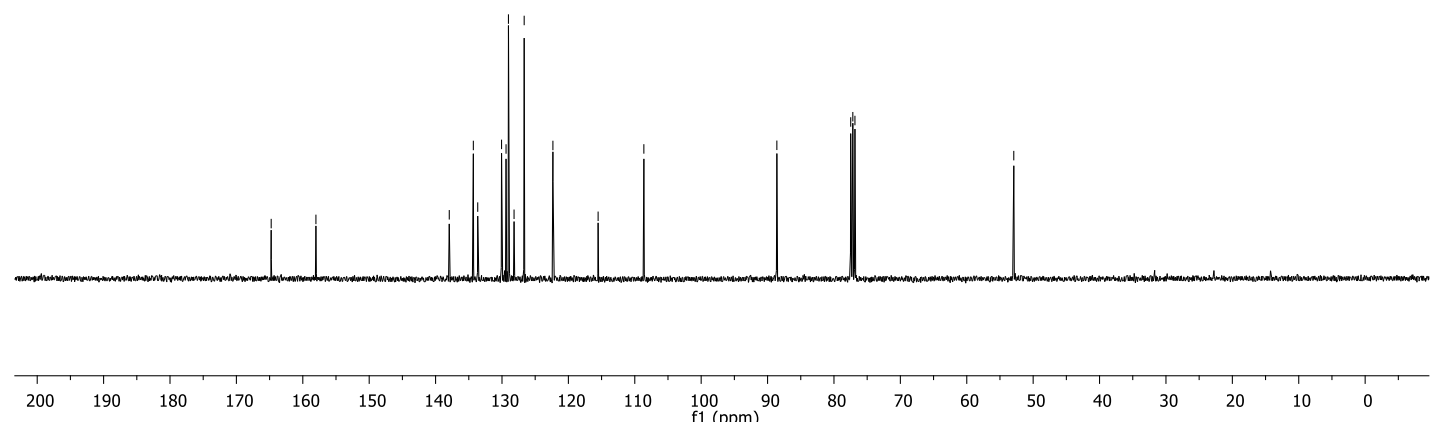


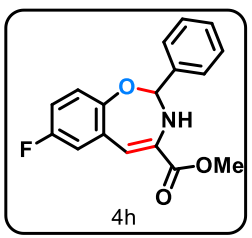

$400 \mathrm{MHz}, \mathrm{CDCl}_{3}$

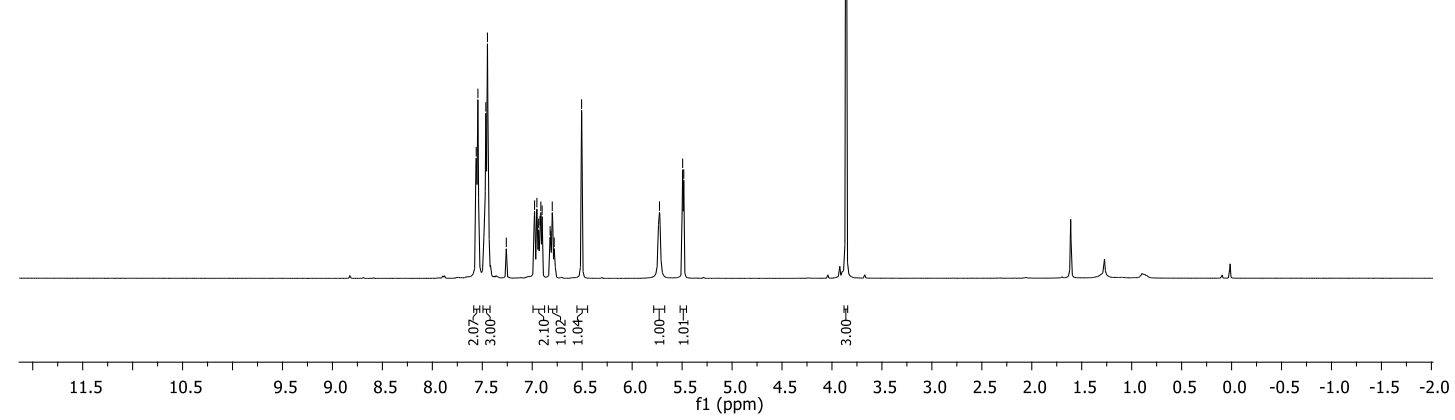

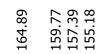

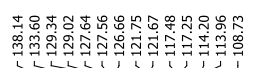

।
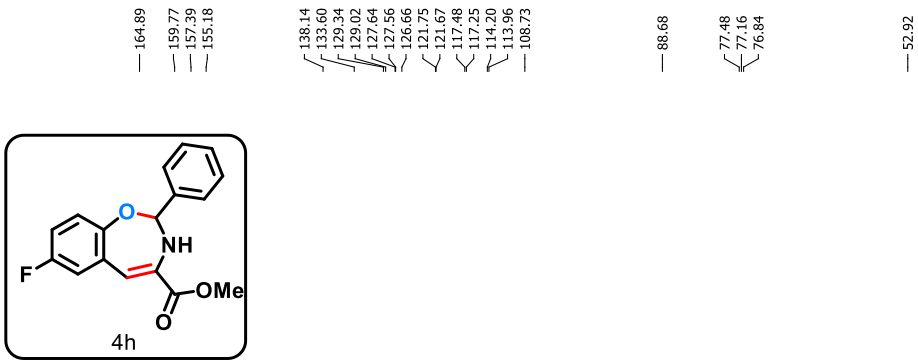

$101 \mathrm{MHz}, \mathrm{CDCl}_{3}$

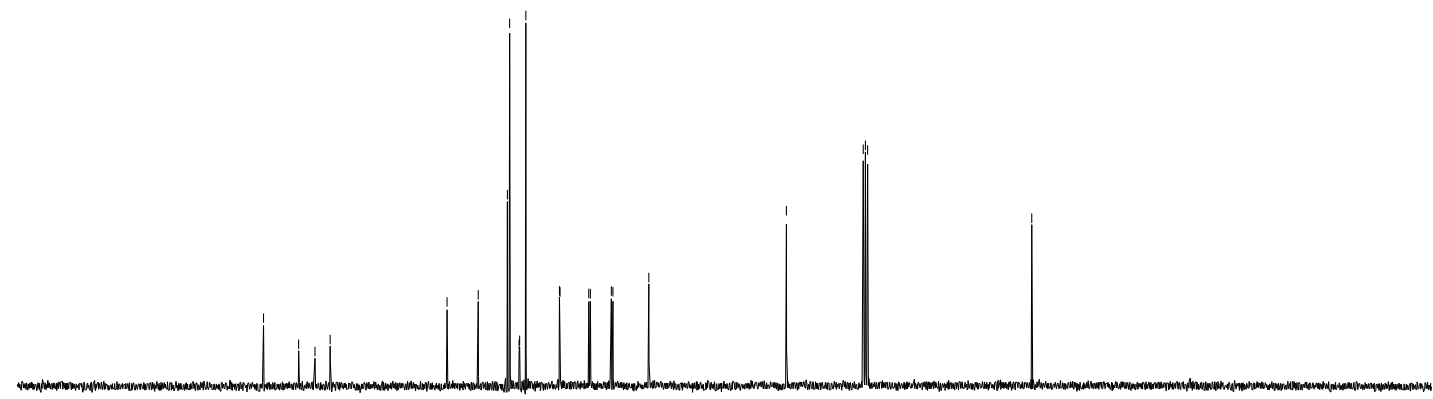

200 


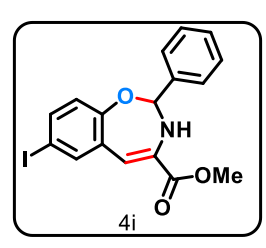

$400 \mathrm{MHz}, \mathrm{CDCl}_{3}$
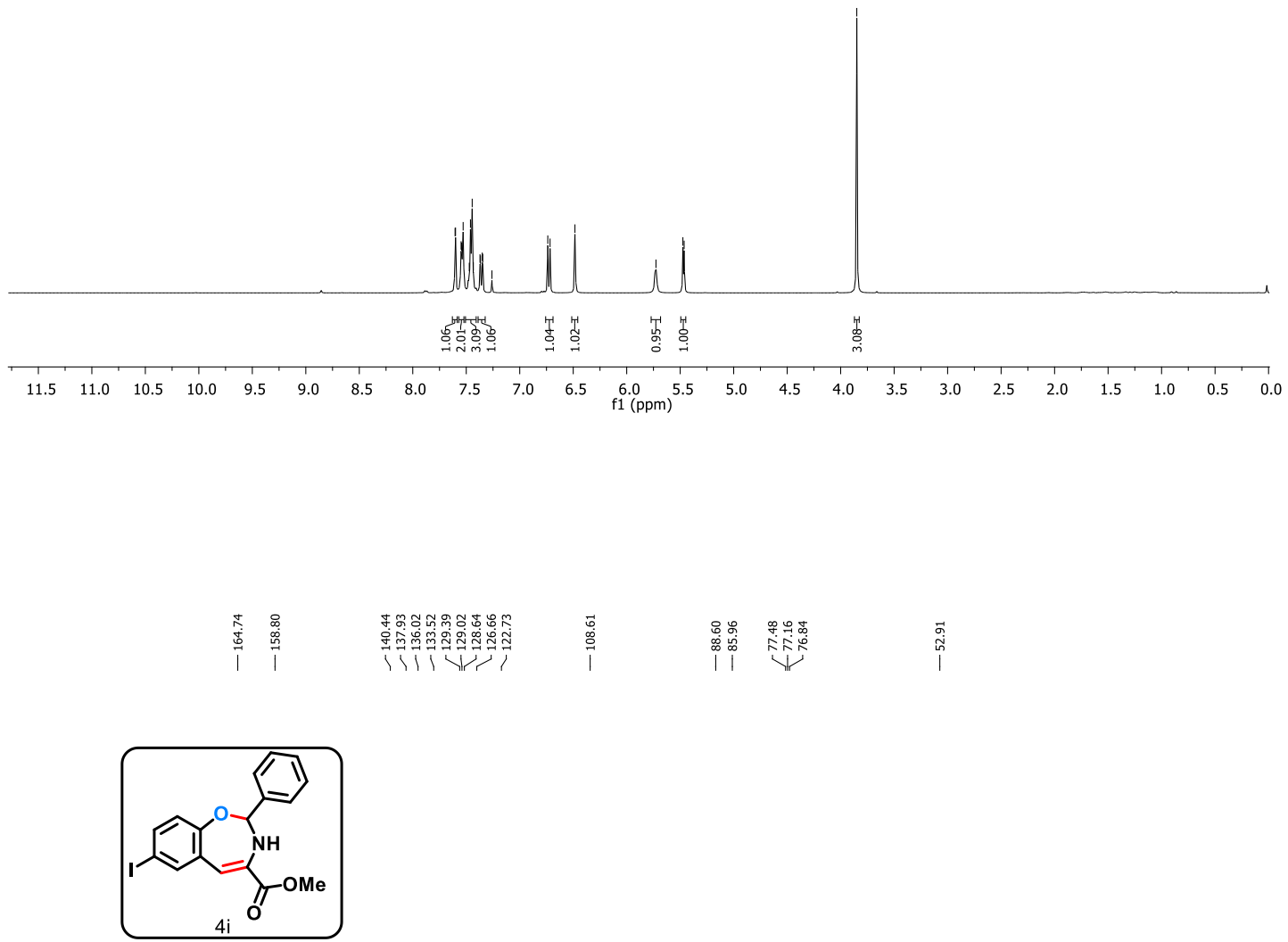

$101 \mathrm{MHz}, \mathrm{CDCl}_{3}$
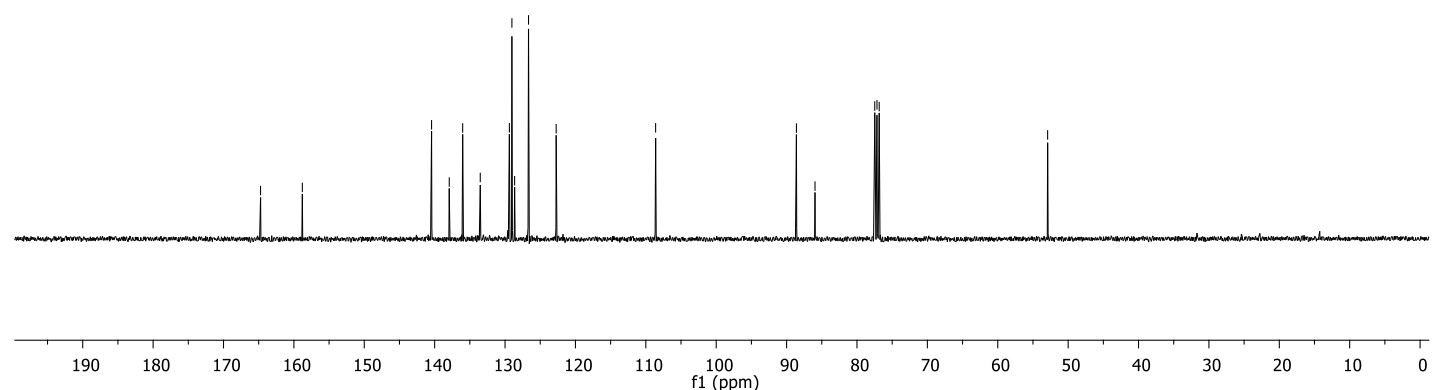


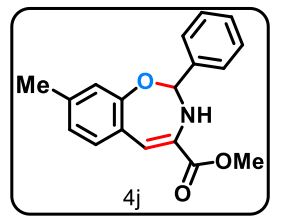

$400 \mathrm{MHz}, \mathrm{CDCl}_{3}$

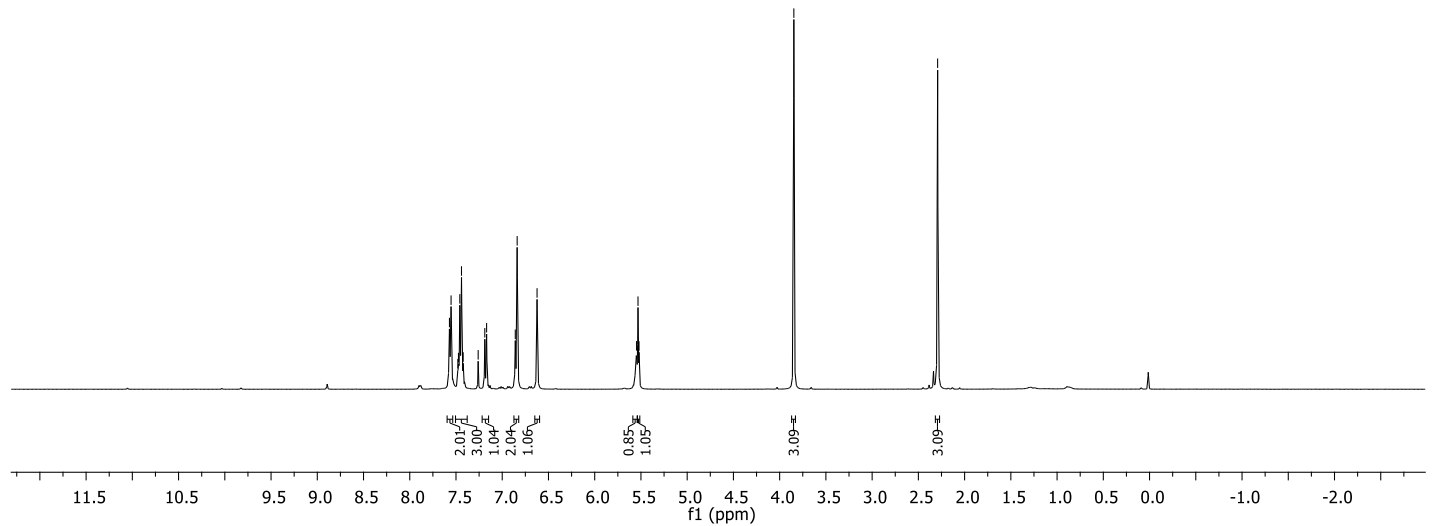

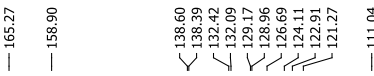

1

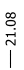

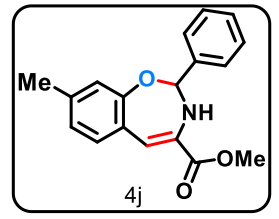

$101 \mathrm{MHz}, \mathrm{CDCl}_{3}$
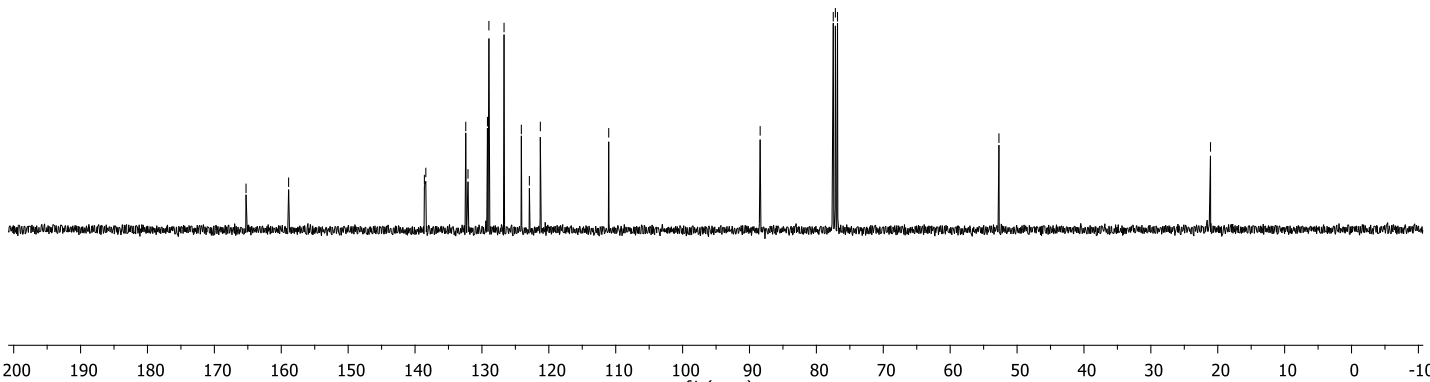


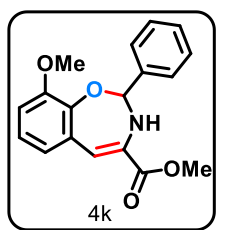

$400 \mathrm{MHz}, \mathrm{CDCl}_{3}$

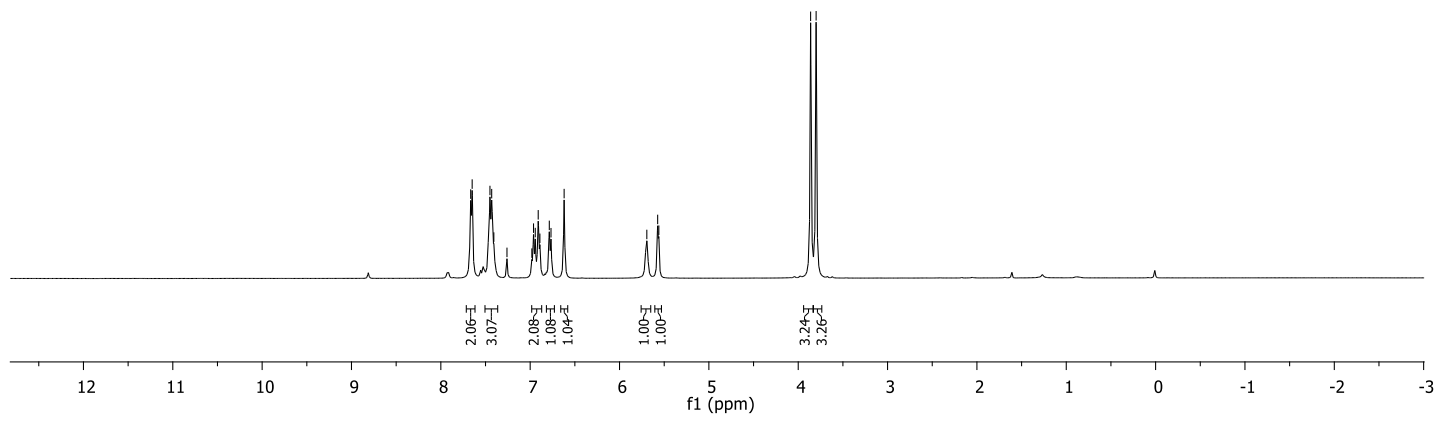

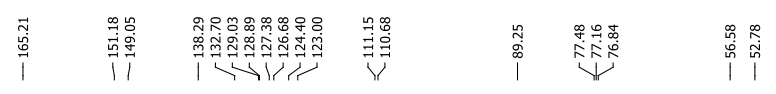

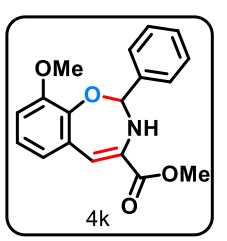

$101 \mathrm{MHz}, \mathrm{CDCl}_{3}$

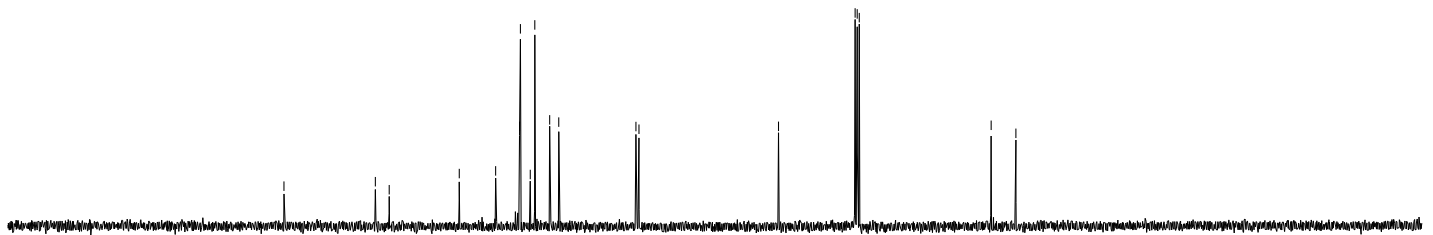

$\begin{array}{lllllllllllllllllllll}200 & 190 & 180 & 170 & 160 & 150 & 140 & 130 & 120 & 110 & 100 & 90 & 80 & 70 & 60 & 50 & 40 & 30 & 20 & 10 & 0\end{array}$ 


\section{|}

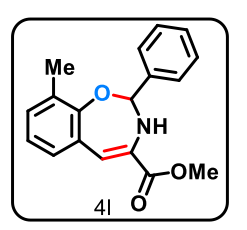

$400 \mathrm{MHz}, \mathrm{CDCl}_{3}$
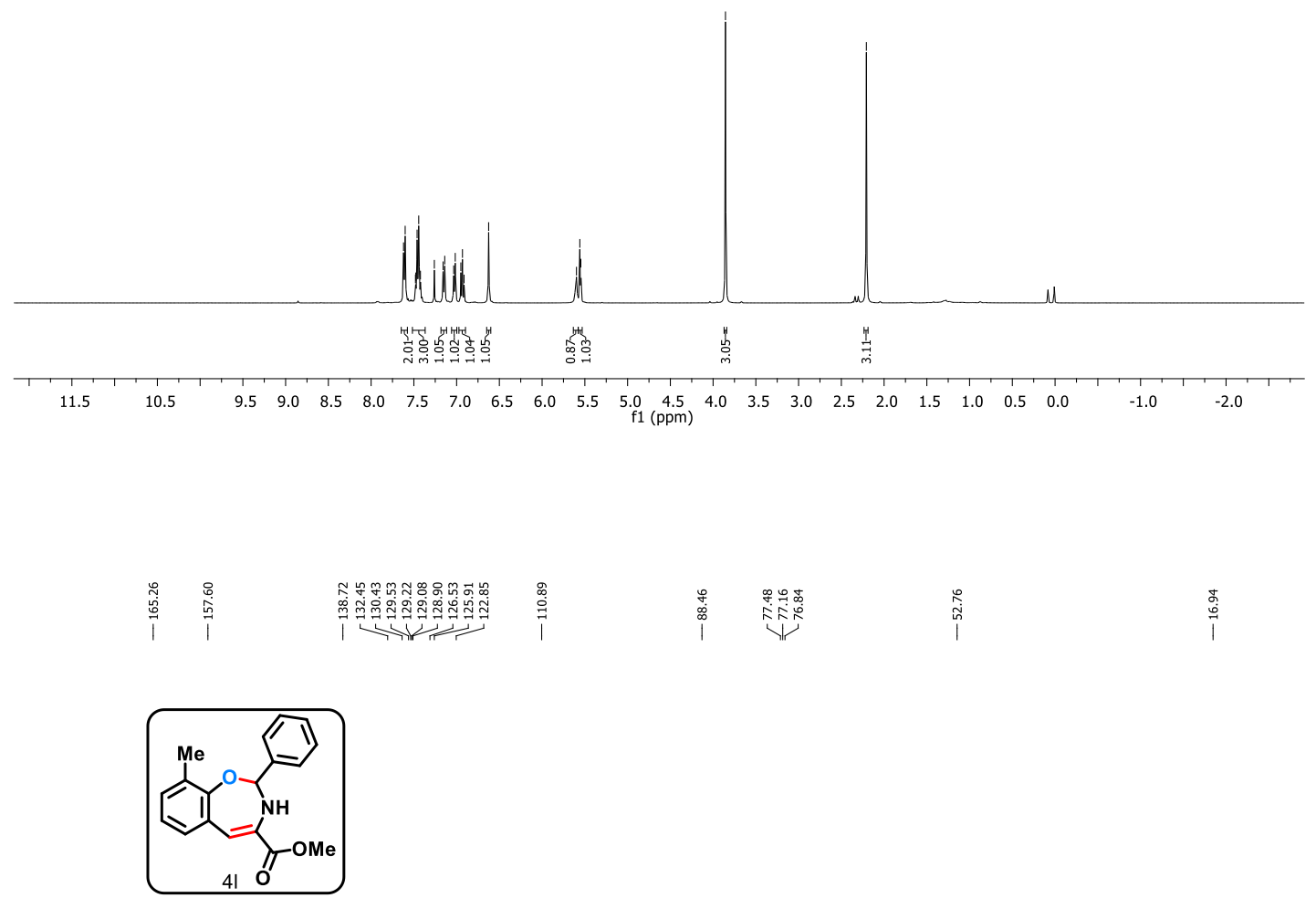

$101 \mathrm{MHz}, \mathrm{CDCl}_{3}$
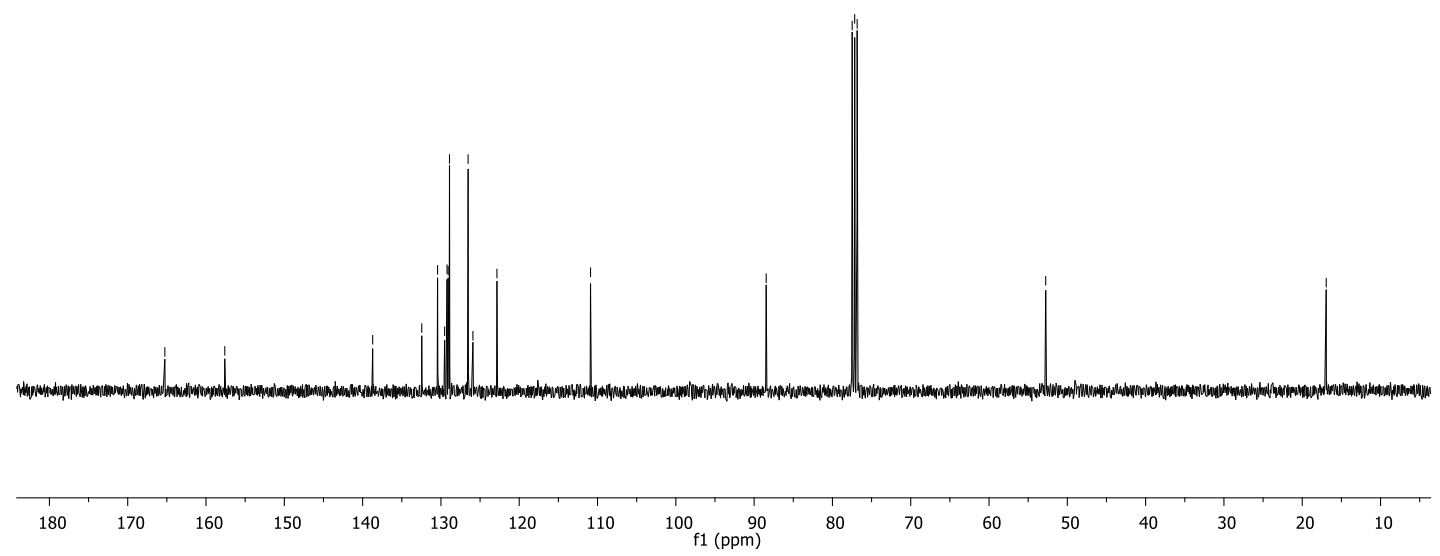


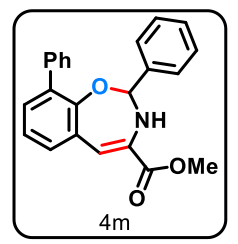

$400 \mathrm{MHz}, \mathrm{CDCl}_{3}$
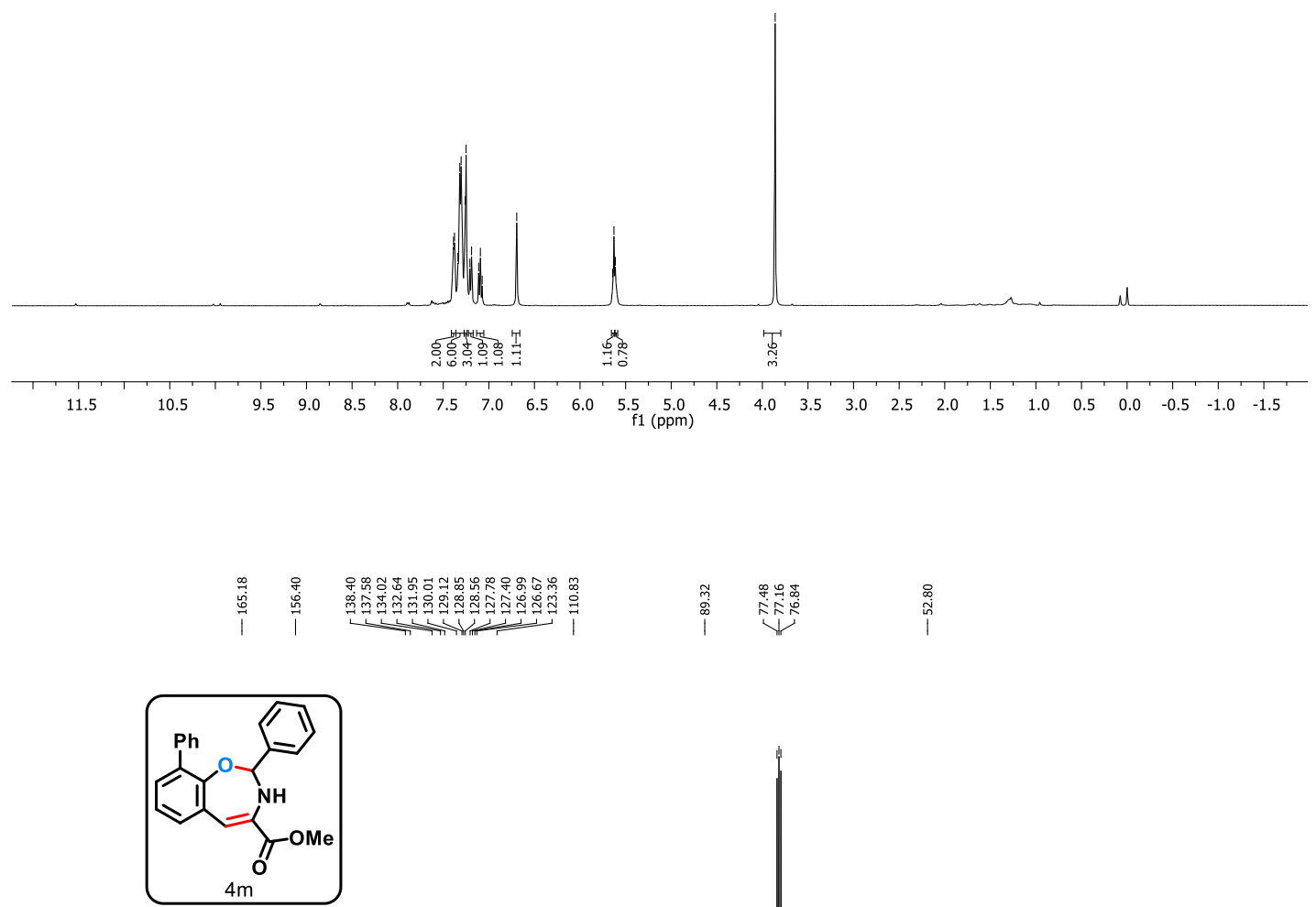

$101 \mathrm{MHz}, \mathrm{CDCl}_{3}$

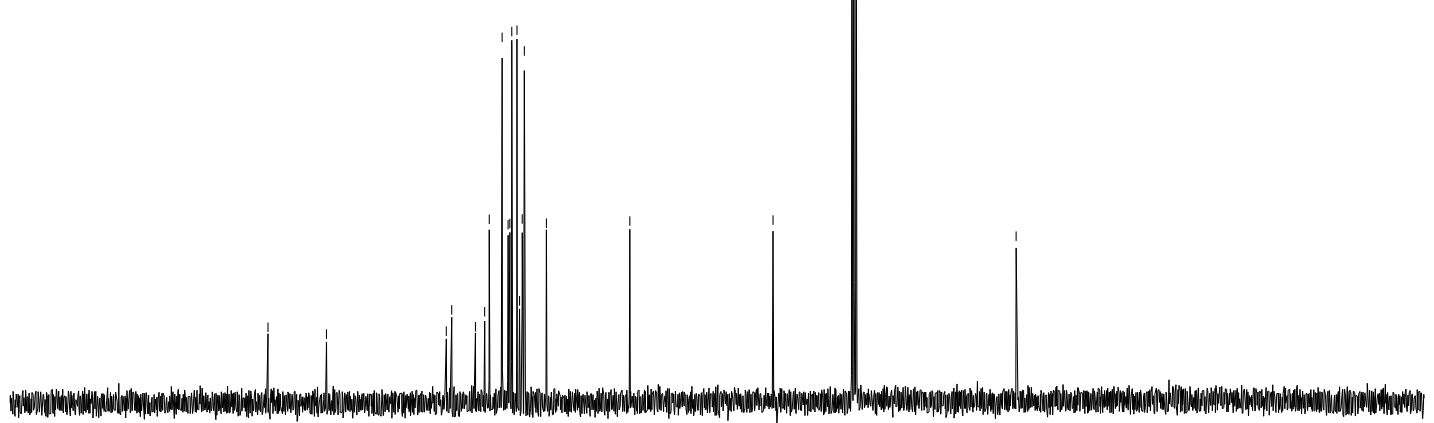

$\begin{array}{lllllllllll}200 & 190 & 180 & 170 & 160 & 150 & 140 & 130 & 120 & 110 & \underset{\mathrm{f} 1(\mathrm{ppm})}{100}\end{array}$ 


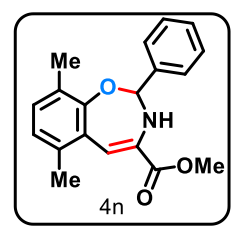

$400 \mathrm{MHz}, \mathrm{CDCl}_{3}$
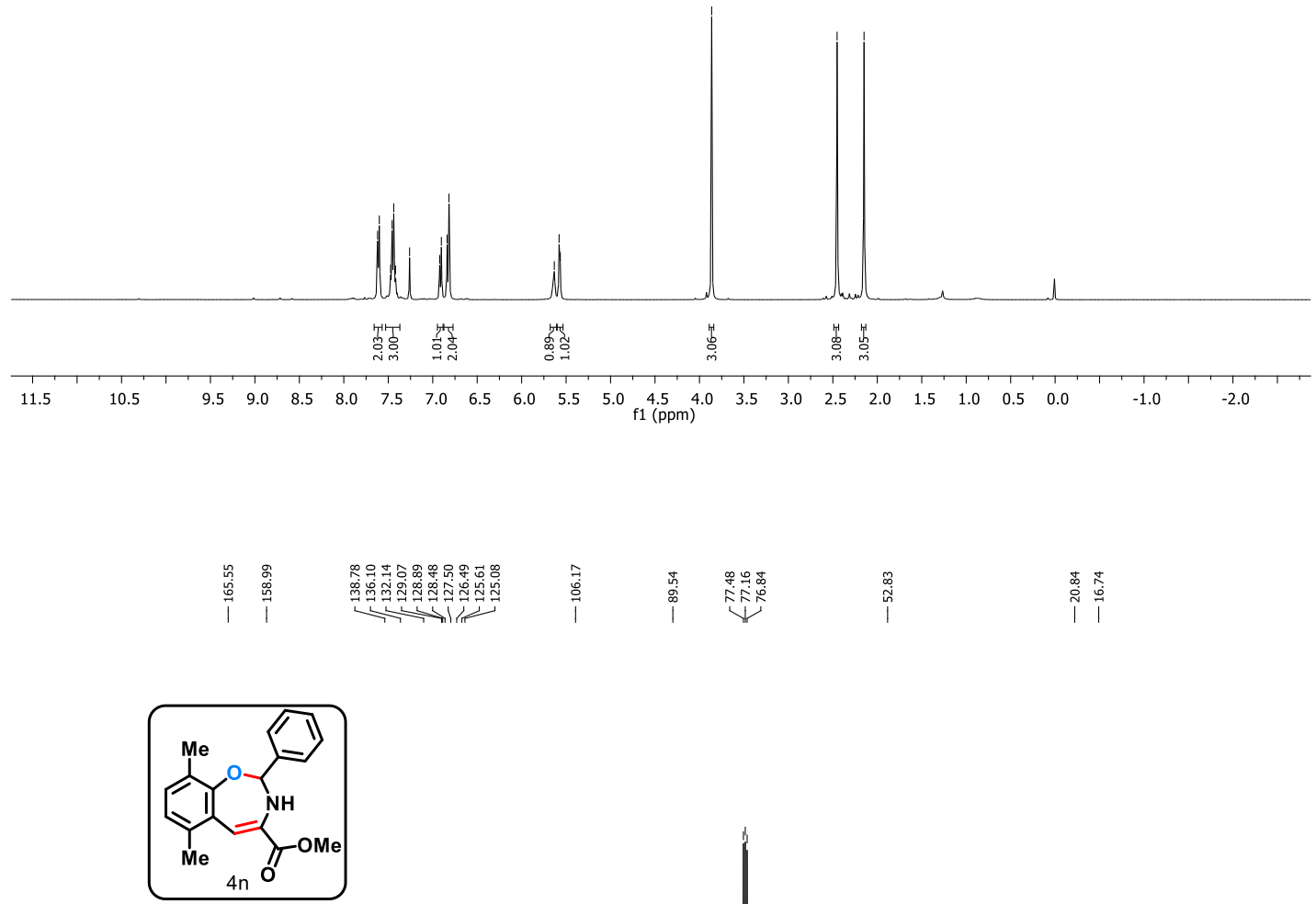

$101 \mathrm{MHz}, \mathrm{CDCl}_{3}$

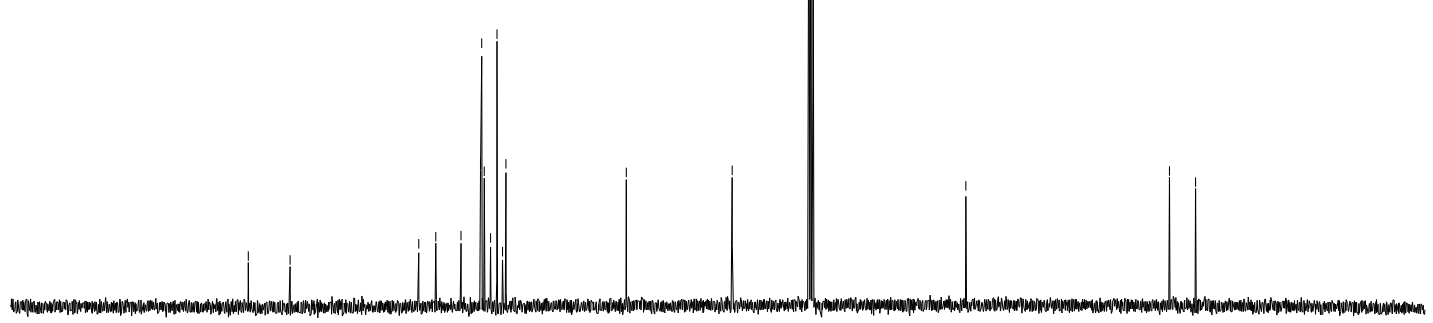

200
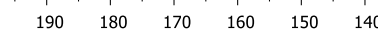

$130 \quad 120$

$00 \begin{gathered}90 \\ f 1(\mathrm{ppm})\end{gathered}$ 


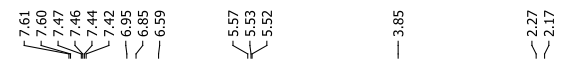

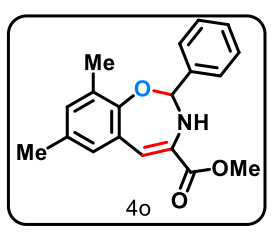

$400 \mathrm{MHz}, \mathrm{CDCl}_{3}$

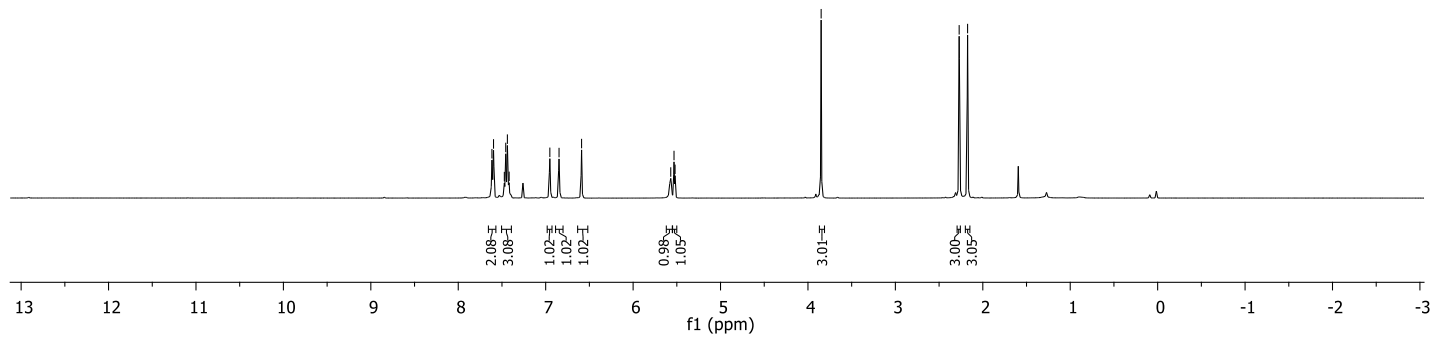

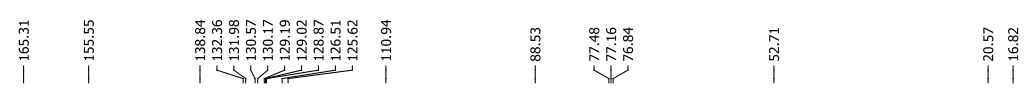

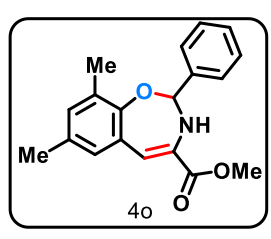

$101 \mathrm{MHz}, \mathrm{CDCl}_{3}$
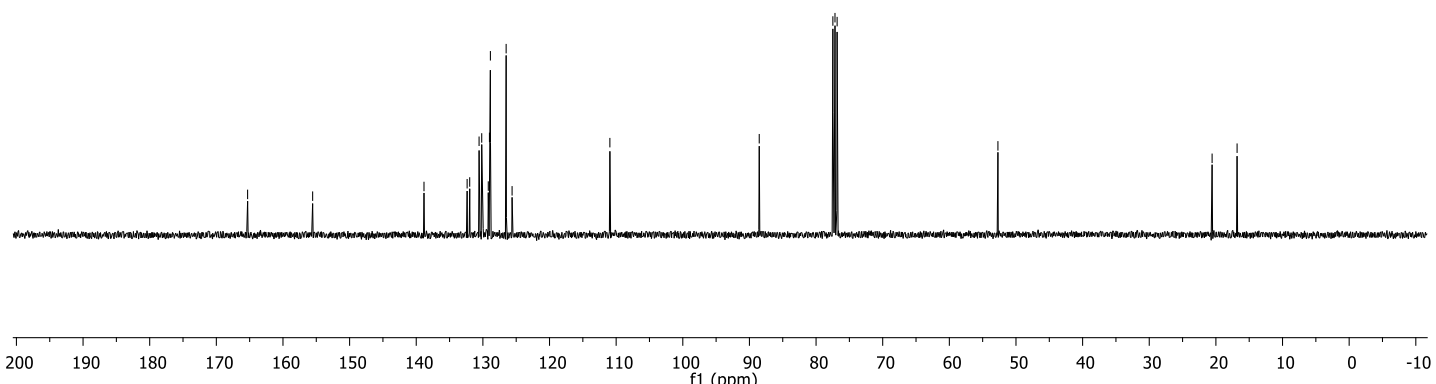


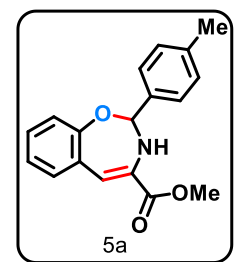

$400 \mathrm{MHz}, \mathrm{CDCl}_{3}$

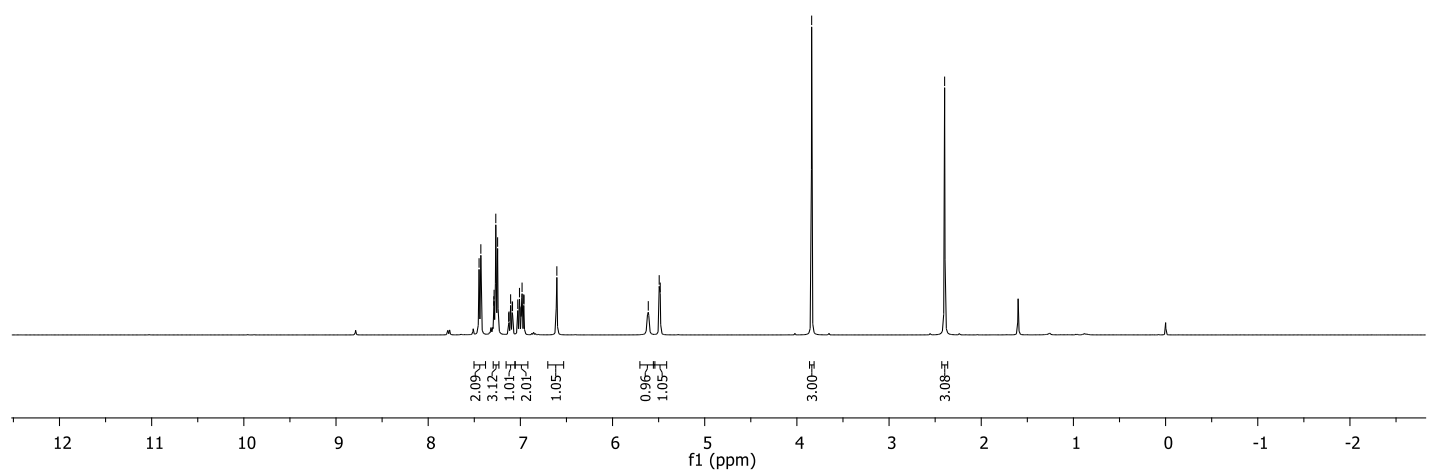

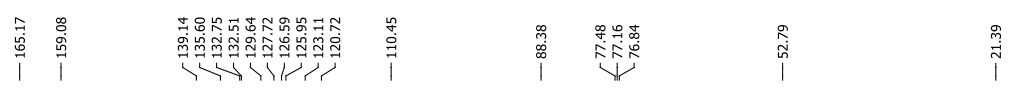

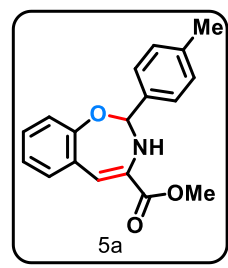

$101 \mathrm{MHz}, \mathrm{CDCl}_{3}$
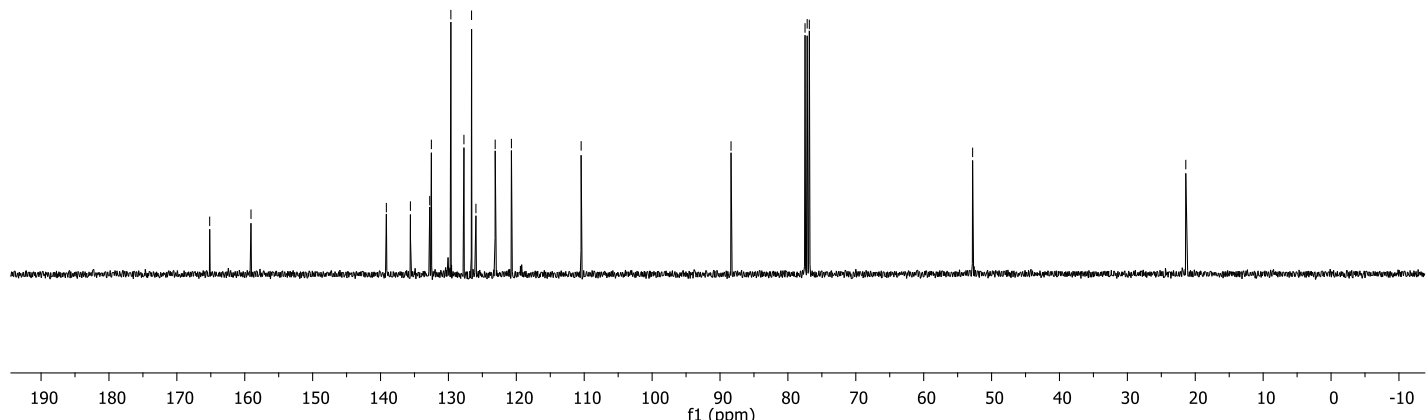

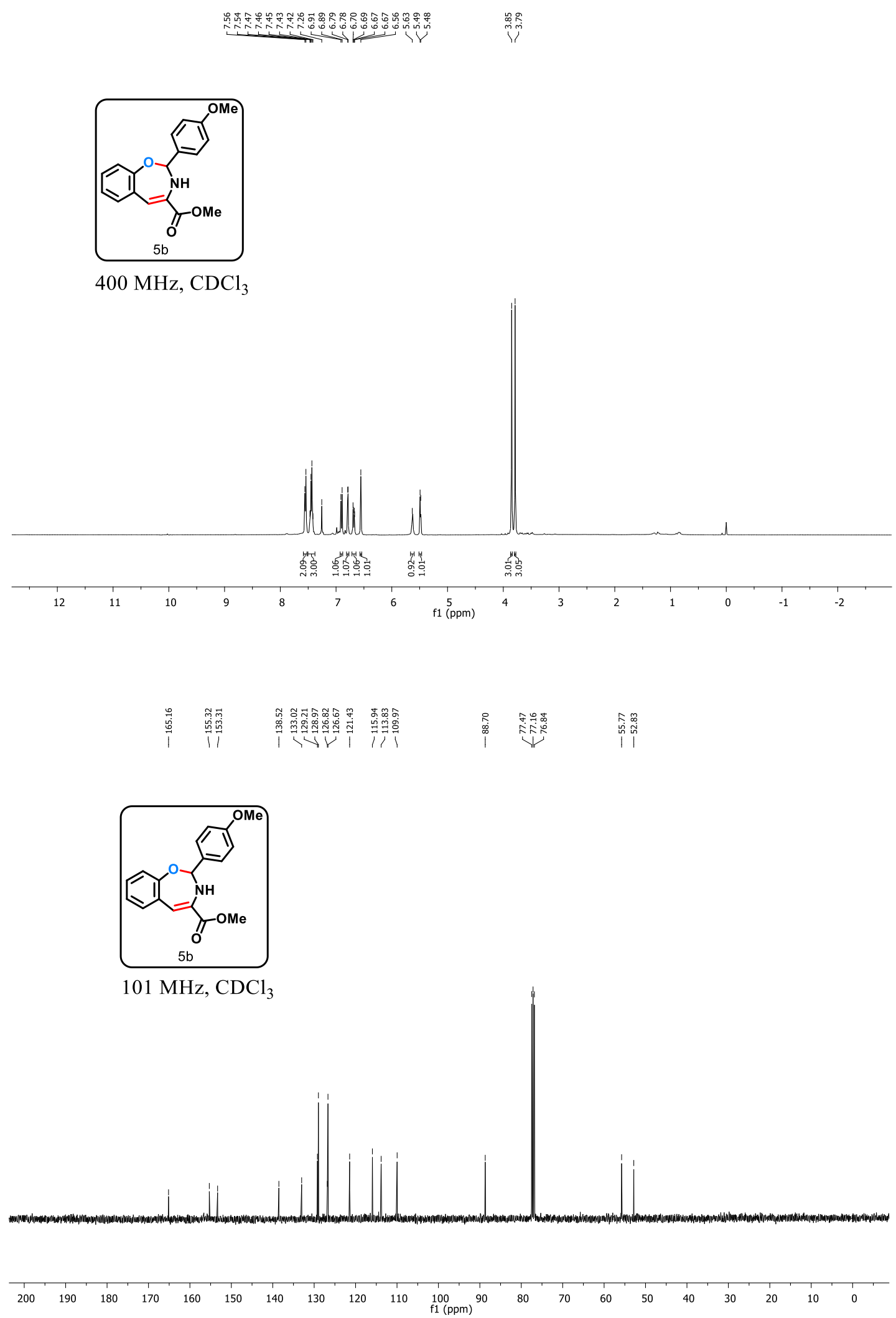


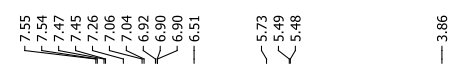

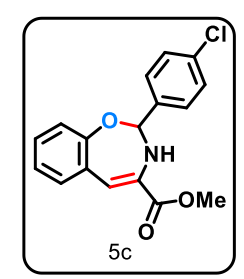

$400 \mathrm{MHz}, \mathrm{CDCl}_{3}$
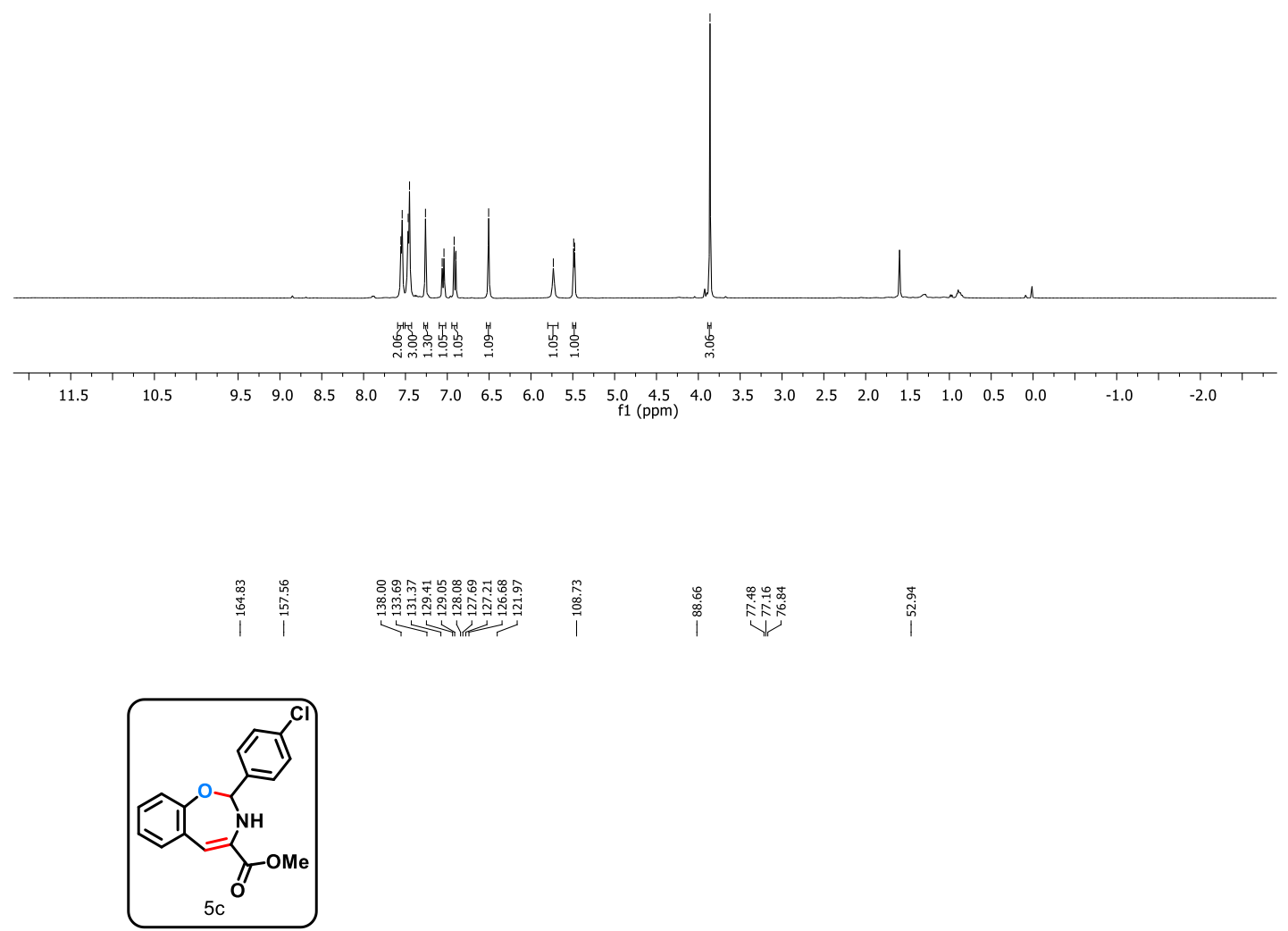

$101 \mathrm{MHz}, \mathrm{CDCl}_{3}$

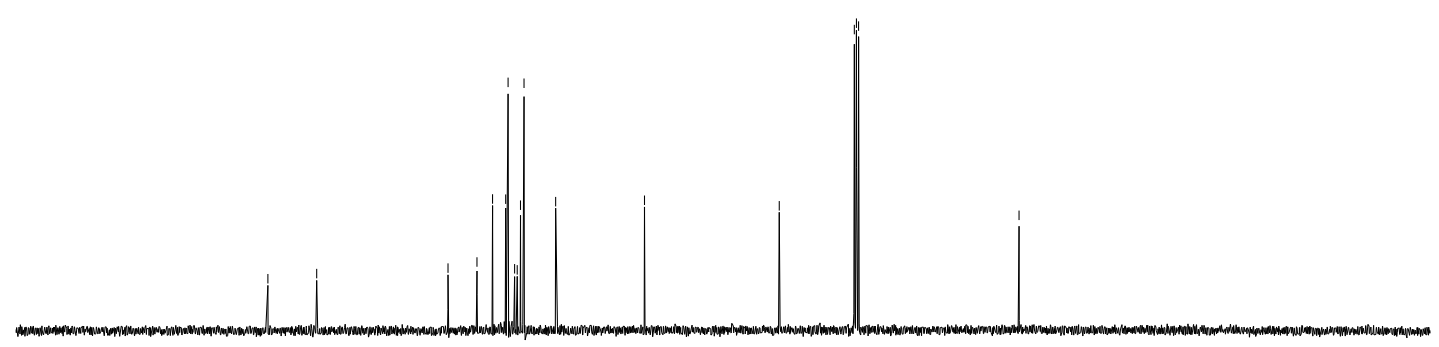

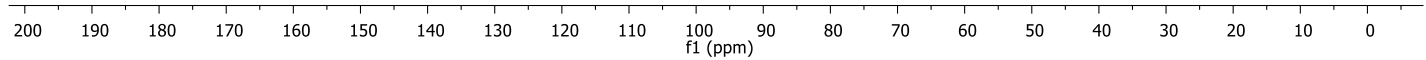




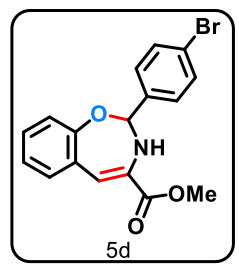

$400 \mathrm{MHz}, \mathrm{CDCl}_{3}$

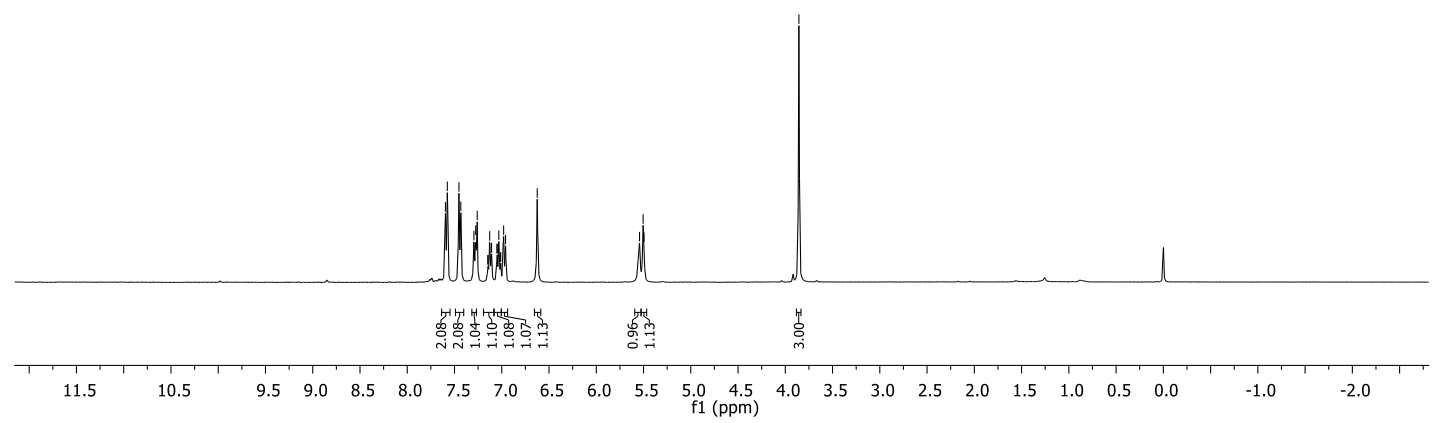

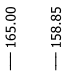

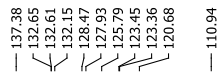

i

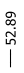

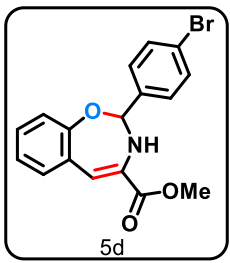

$101 \mathrm{MHz}, \mathrm{CDCl}_{3}$

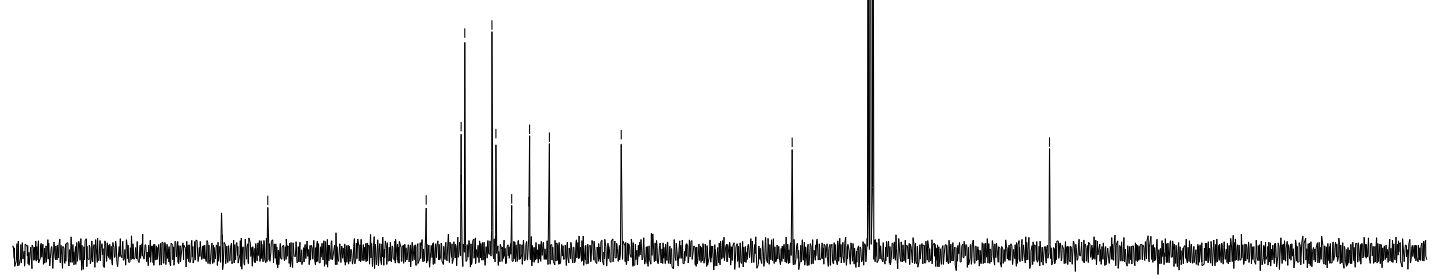

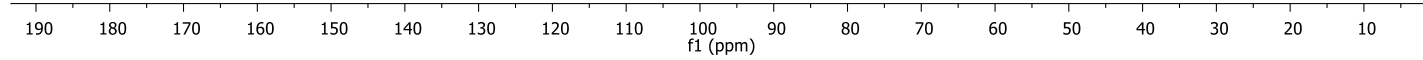




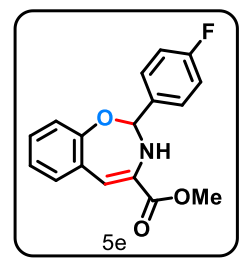

$400 \mathrm{MHz}, \mathrm{CDCl}_{3}$
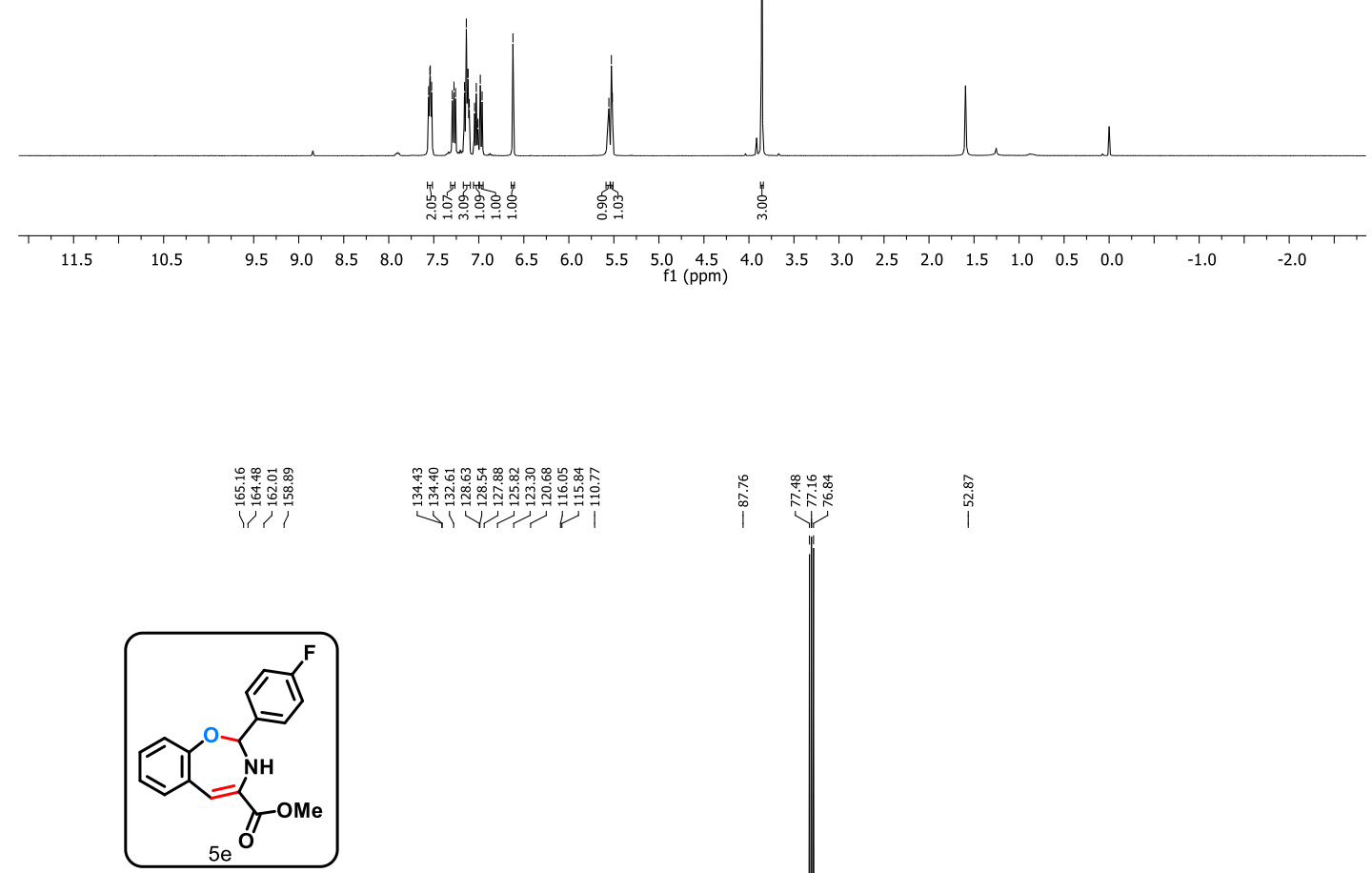

$101 \mathrm{MHz}, \mathrm{CDCl}_{3}$

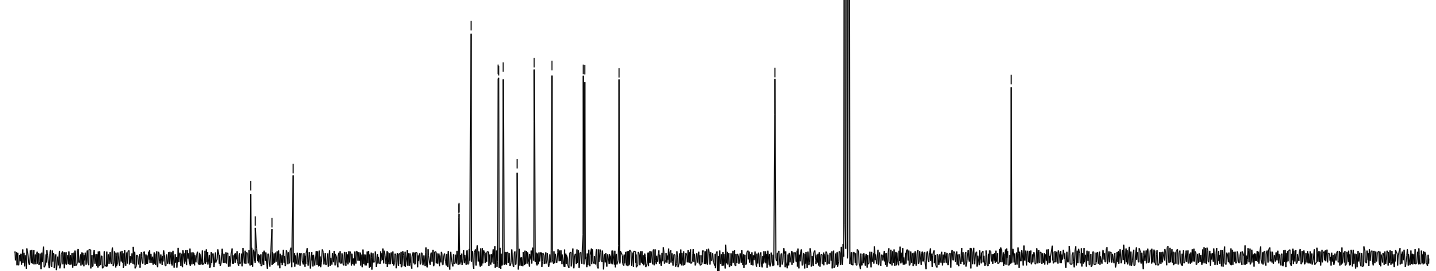

$\begin{array}{llllllllll}190 & 180 & 170 & 160 & 150 & 140 & 130 & 120 & 110 & 100 \\ 100 & \end{array}$ 

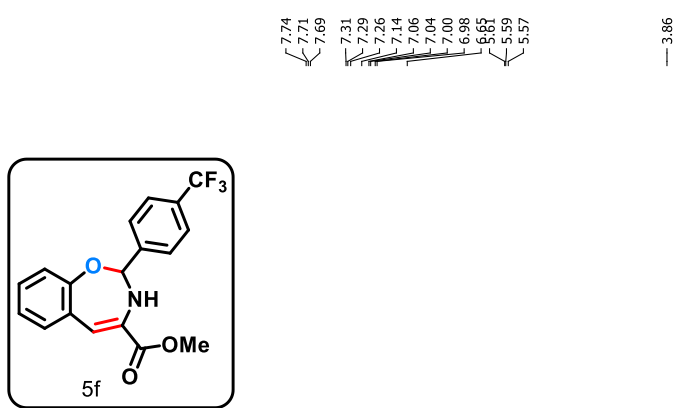

$400 \mathrm{MHz}, \mathrm{CDCl}_{3}$

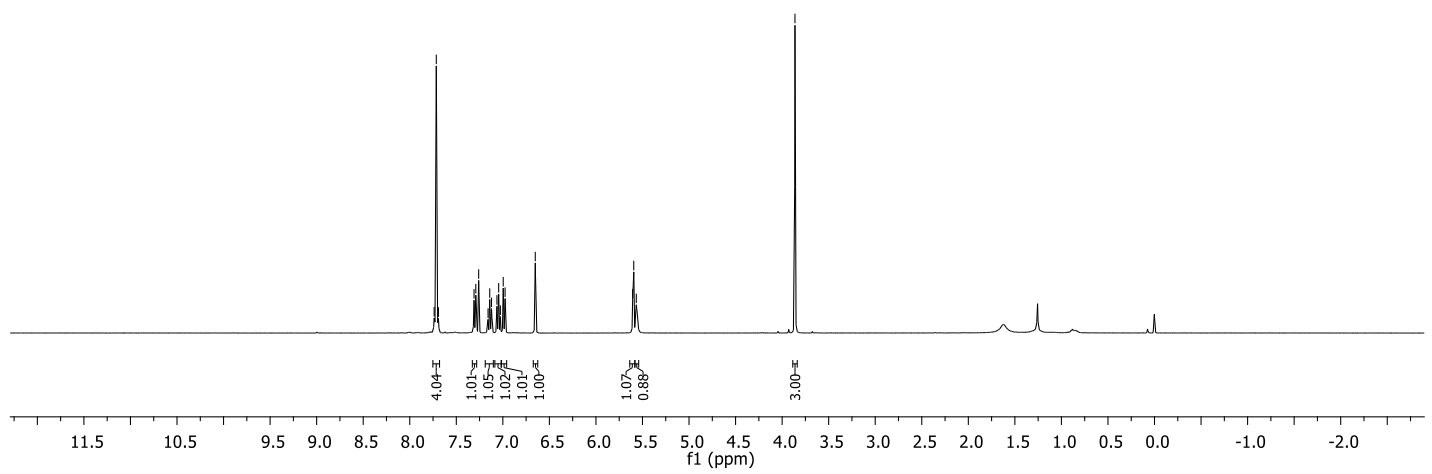

|

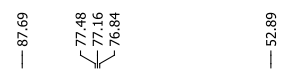

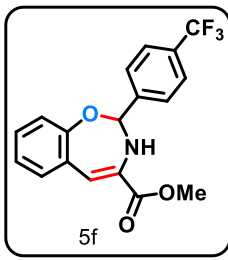

$101 \mathrm{MHz}, \mathrm{CDCl}_{3}$
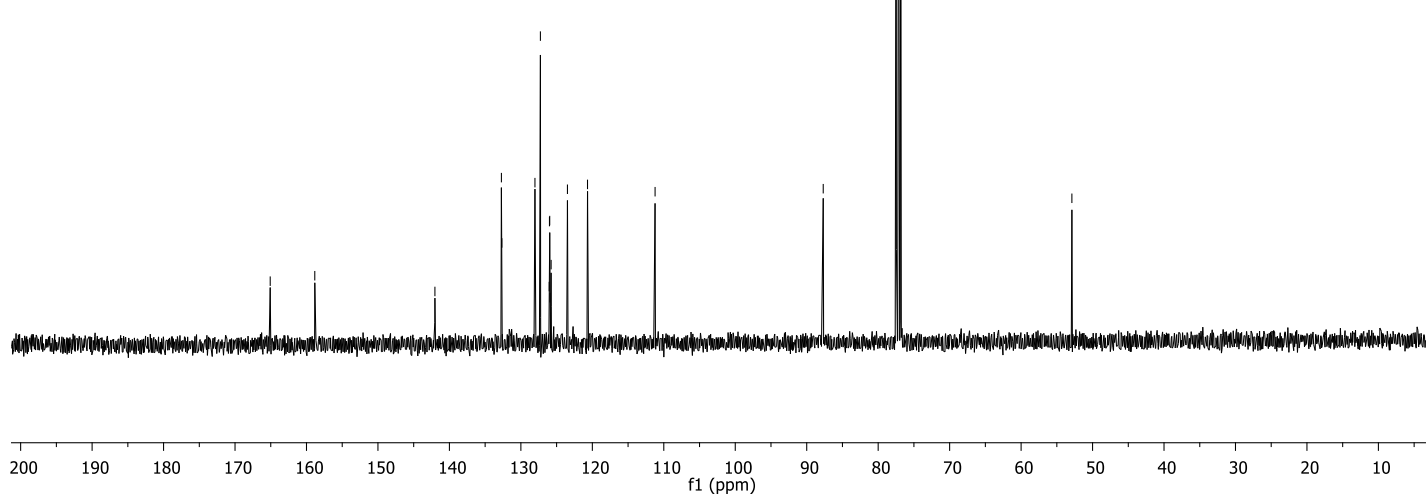

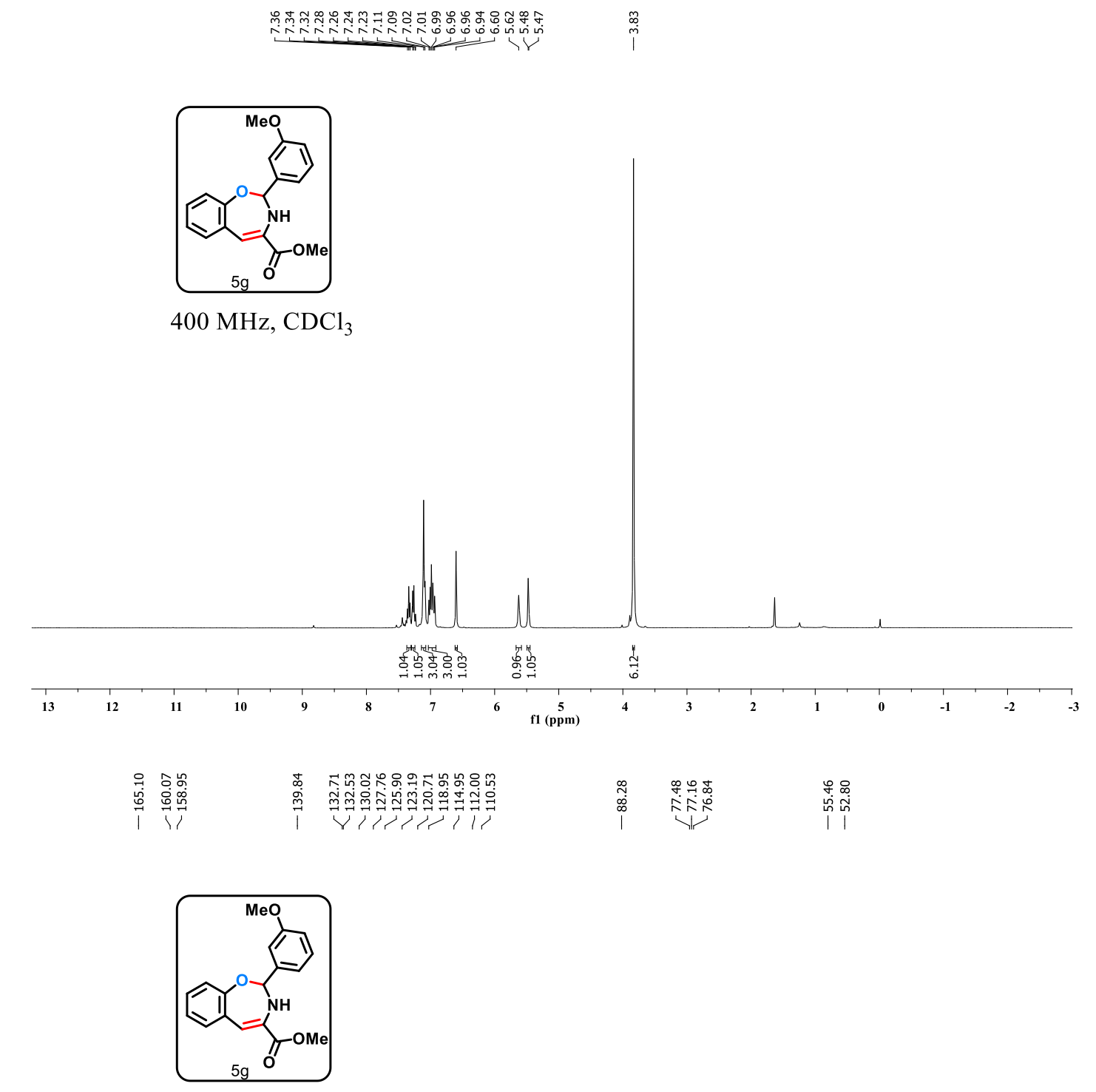

$101 \mathrm{MHz}, \mathrm{CDCl}_{3}$

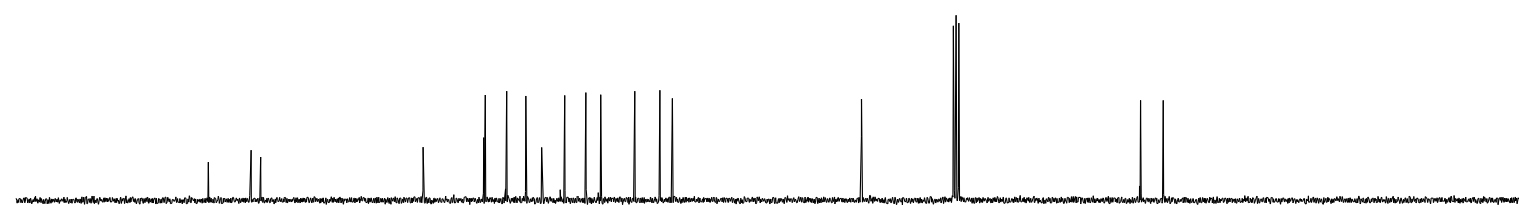




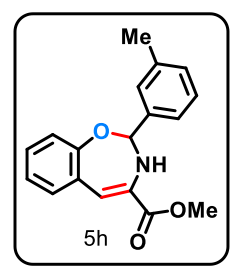

$400 \mathrm{MHz}, \mathrm{CDCl}_{3}$

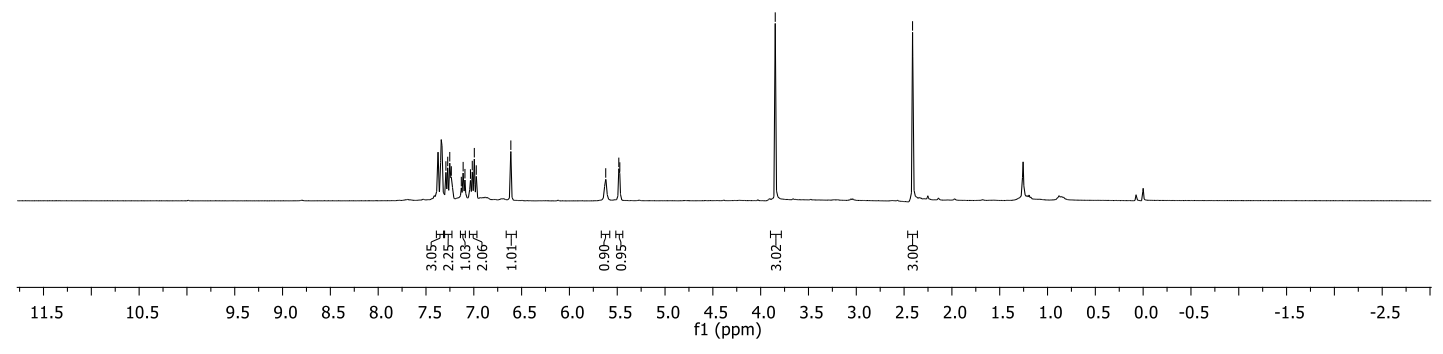

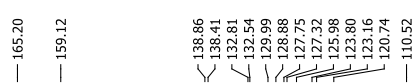

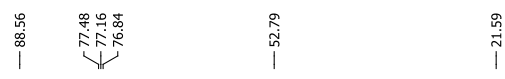

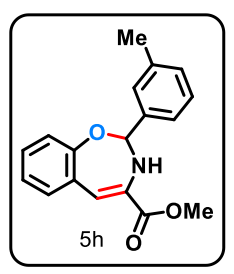

$101 \mathrm{MHz}, \mathrm{CDCl}_{3}$

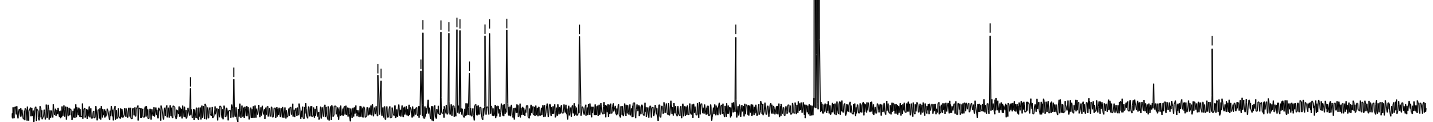

$\begin{array}{llllllllllllllllllll}190 & 180 & 170 & 160 & 150 & 140 & 130 & 120 & 110 & 100 & 90 & 80 & 70 & 60 & 50 & 40 & 30 & 20 & 10 & 0\end{array}$ 


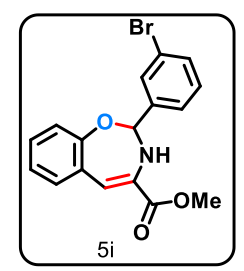

$400 \mathrm{MHz}, \mathrm{CDCl}_{3}$

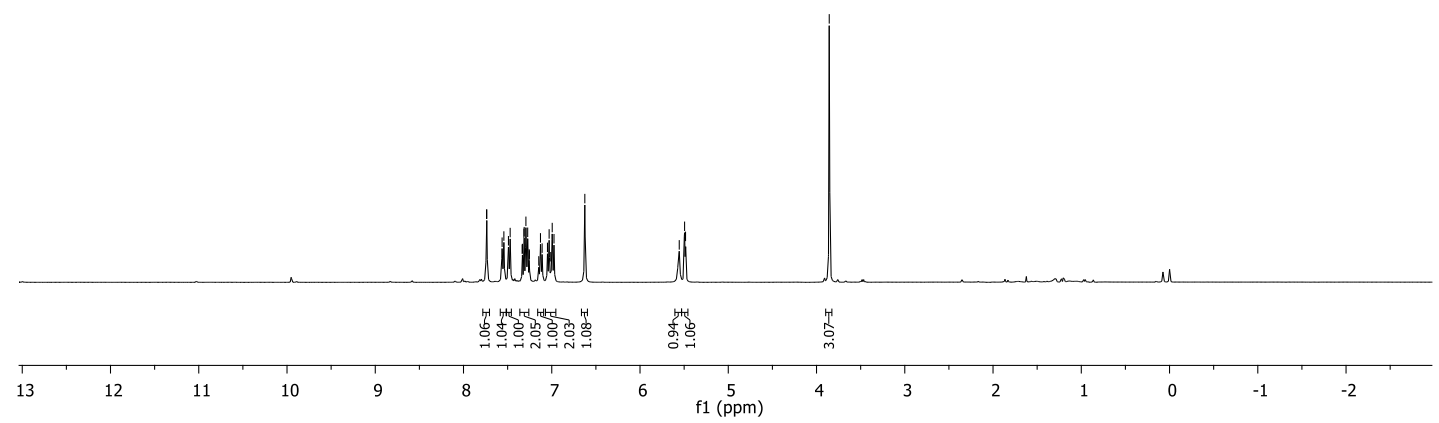

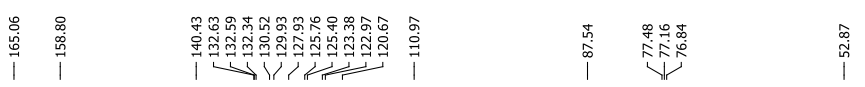

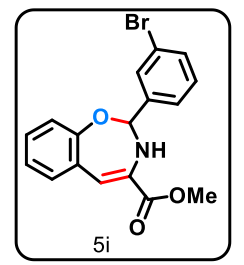

$101 \mathrm{MHz}, \mathrm{CDCl}_{3}$
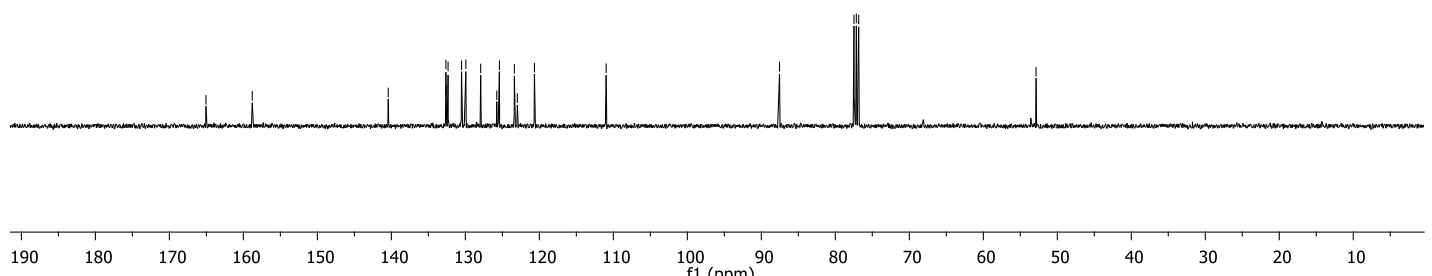

${ }_{\mathrm{f} 1(\mathrm{ppm})}{ }^{90}$ 


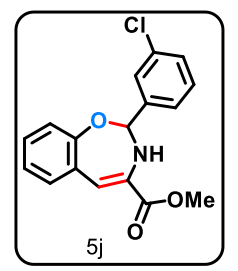

$400 \mathrm{MHz}, \mathrm{CDCl}_{3}$

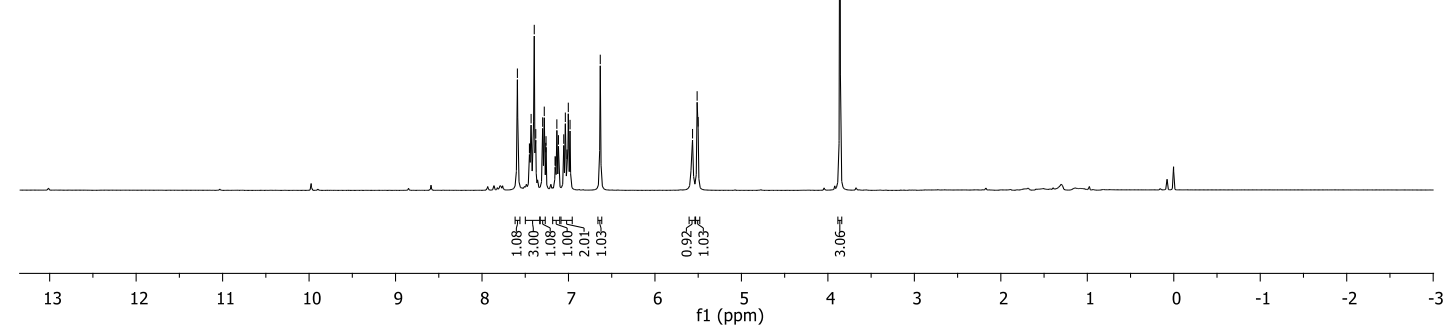

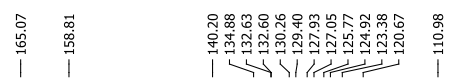

1)

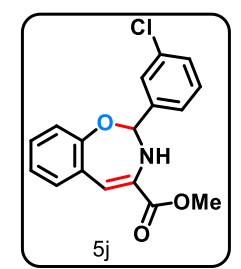

$101 \mathrm{MHz}, \mathrm{CDCl}_{3}$
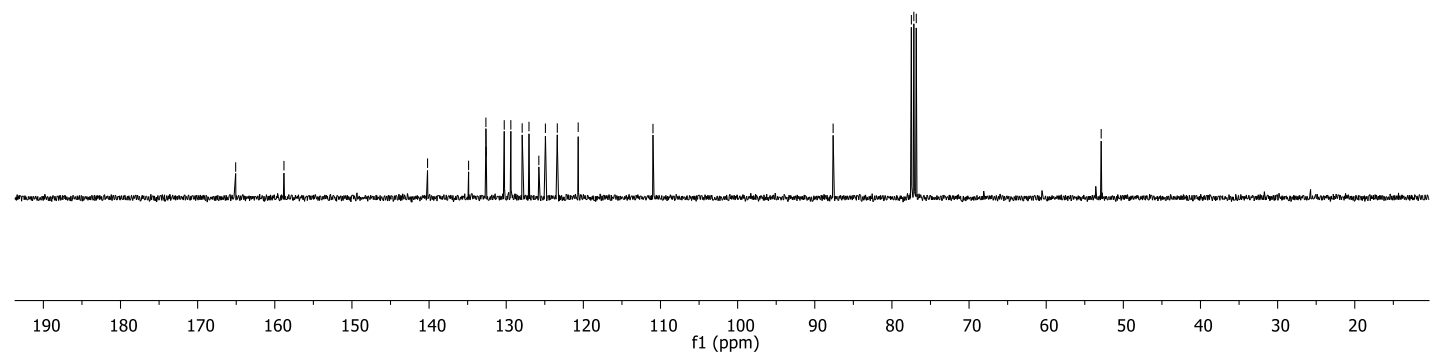

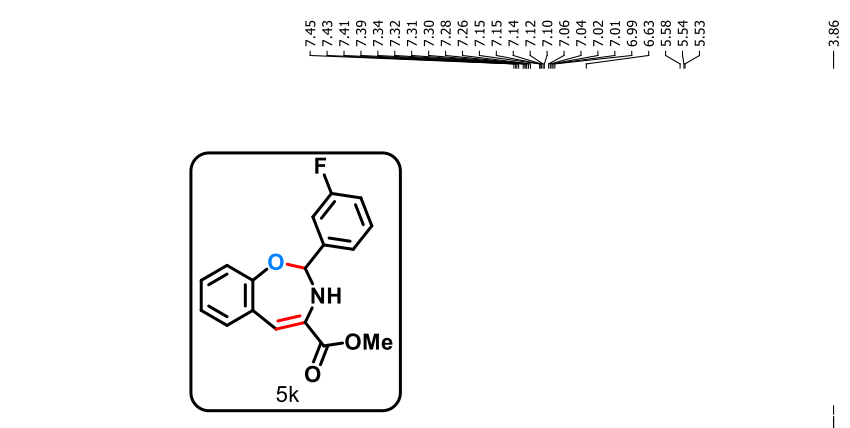

$400 \mathrm{MHz}, \mathrm{CDCl}_{3}$
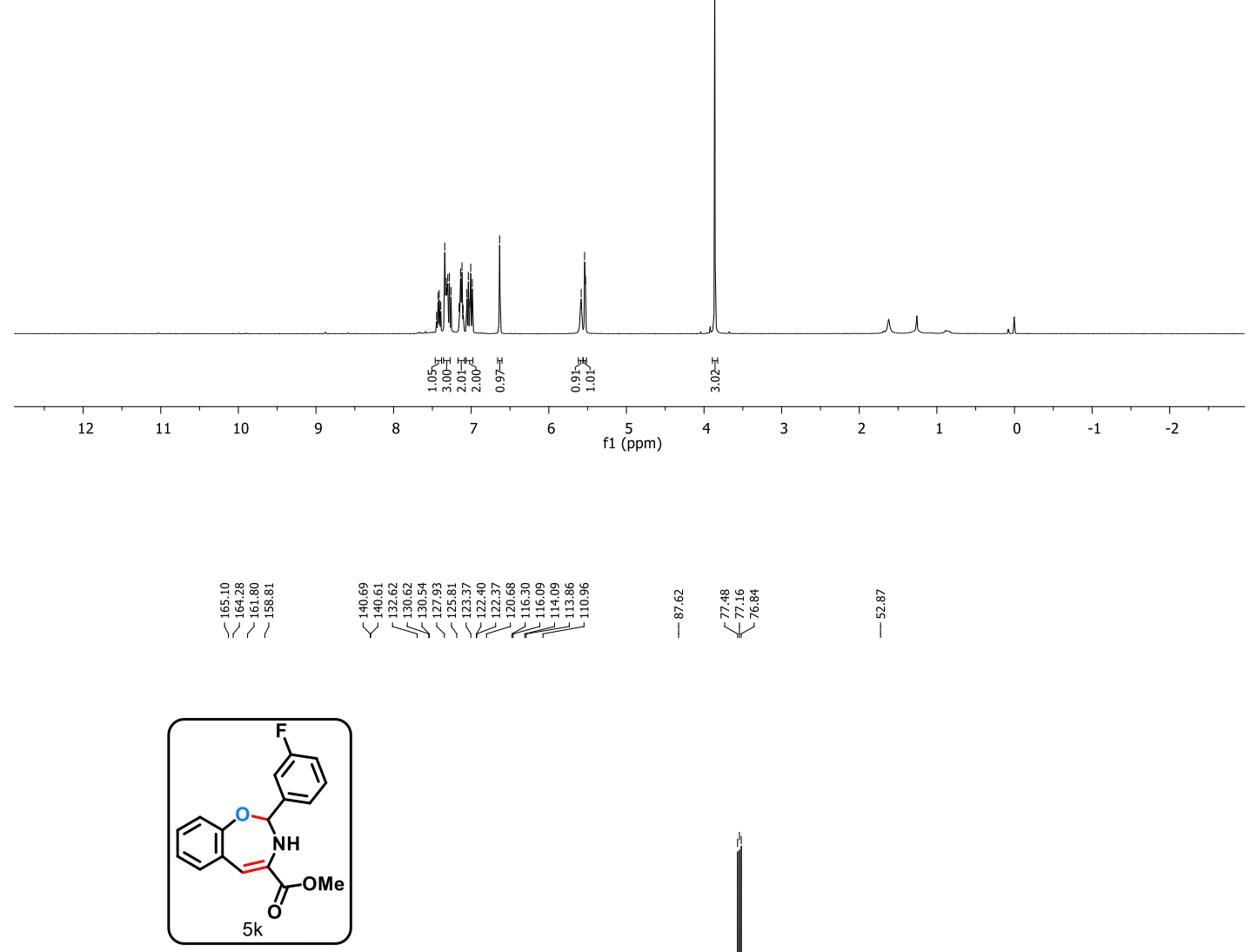

$101 \mathrm{MHz}, \mathrm{CDCl}_{3}$
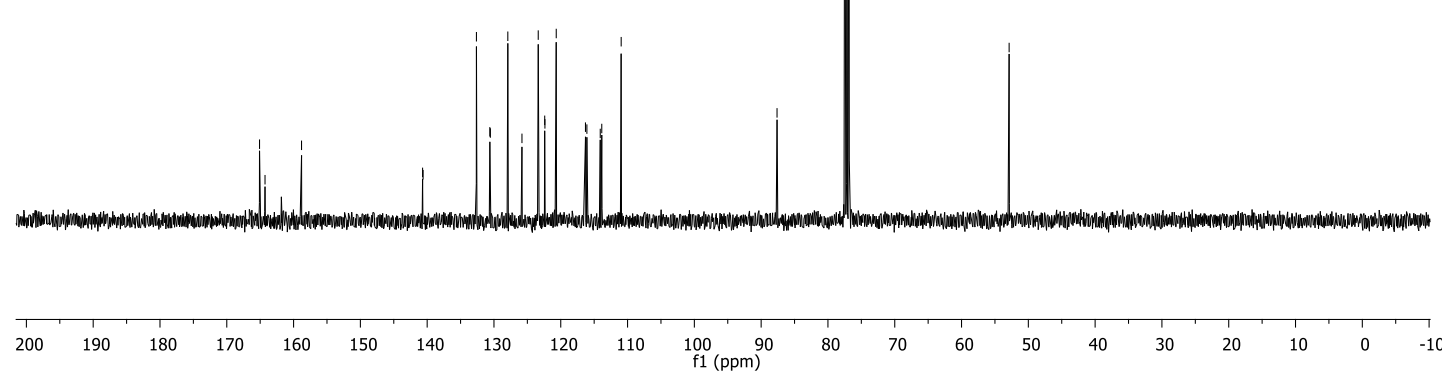


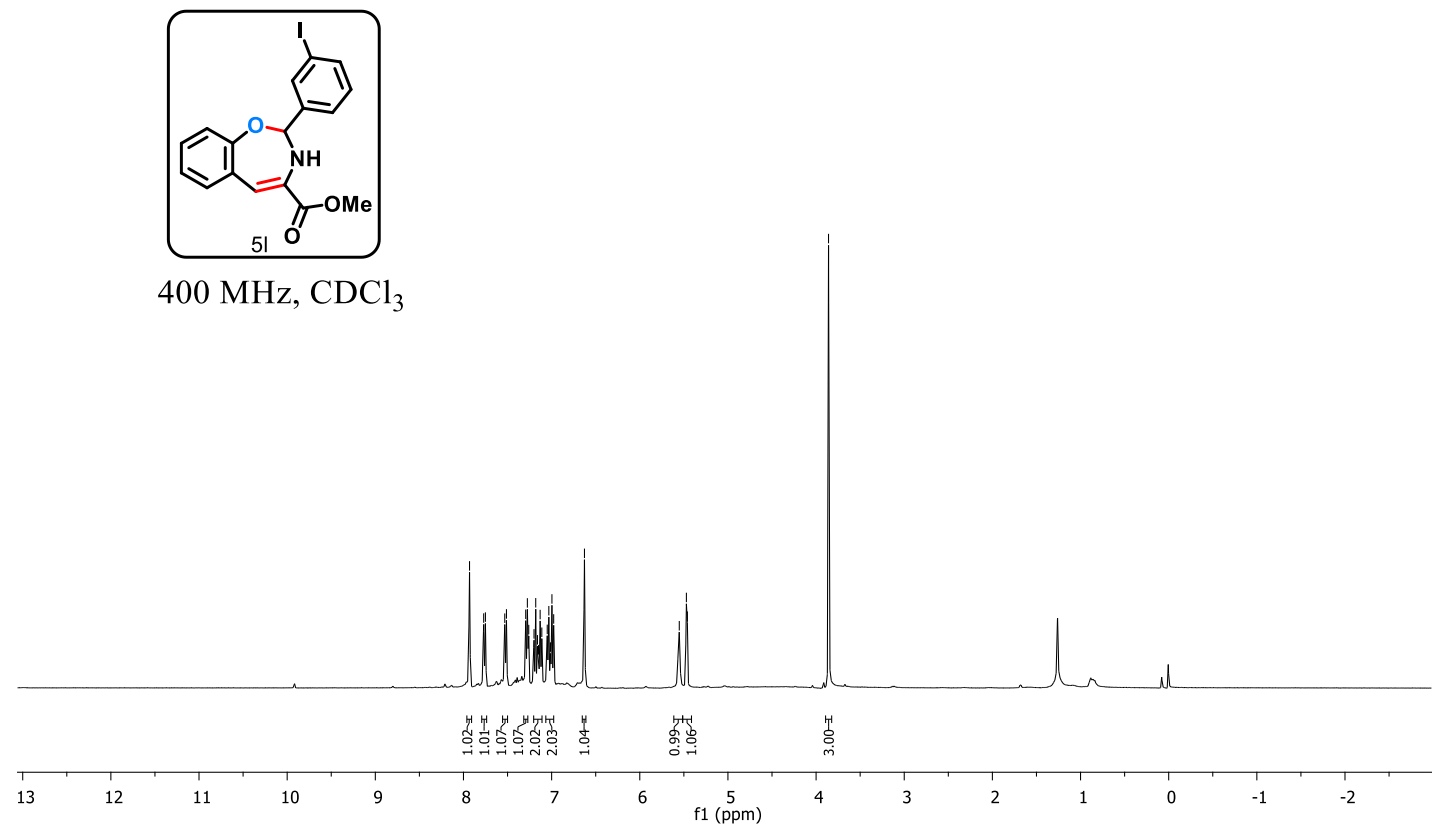

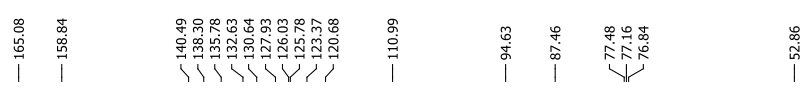

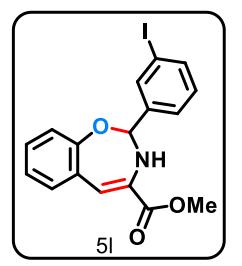

$101 \mathrm{MHz}, \mathrm{CDCl}_{3}$

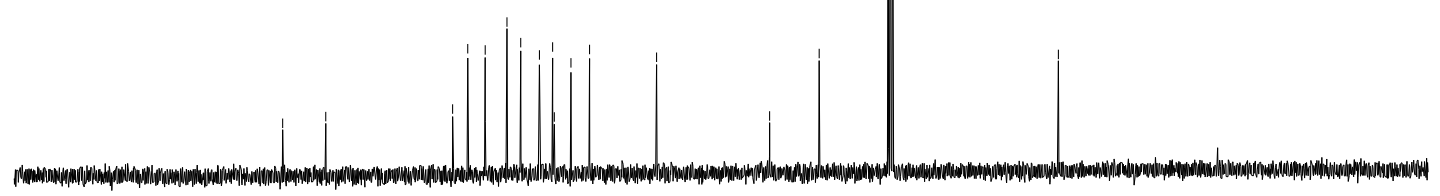

$\begin{array}{lllllllllllllllllllll}200 & 190 & 180 & 170 & 160 & 150 & 140 & 130 & 120 & 110 & \begin{array}{l}100 \\ \mathrm{f} 1(\mathrm{ppm})\end{array} & 90 & 80 & 70 & 60 & 50 & 40 & 30 & 20 & 10 & 0\end{array}$ 

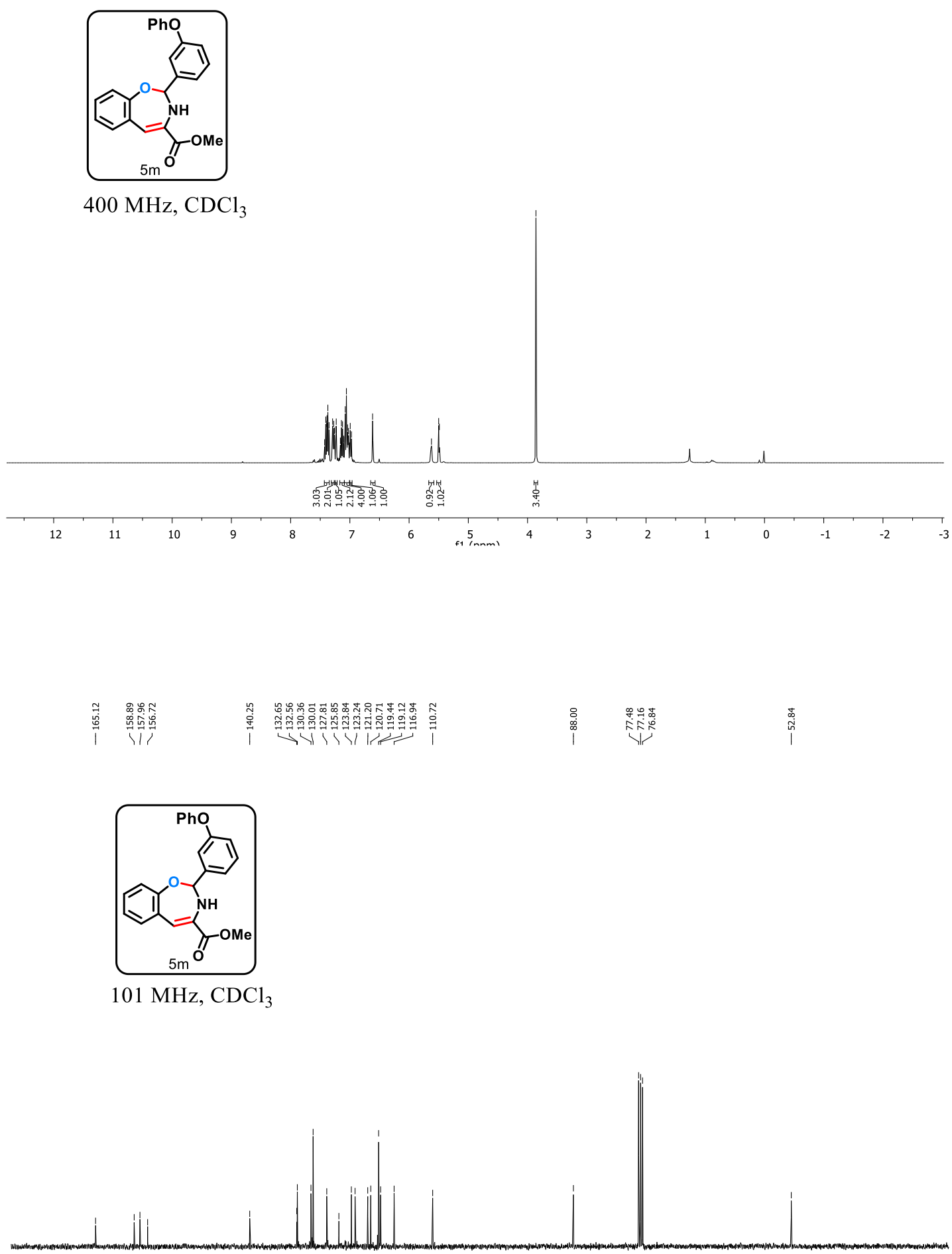

$\begin{array}{lllllllllllllllllllllllllllllllllll}175 & 170 & 165 & 160 & 155 & 150 & 145 & 140 & 135 & 130 & 125 & 120 & 115 & 110 & 105 & 100 & 95 & 90 & 85 & 80 & 75 & 70 & 65 & 60 & 55 & 50 & 45 & 40 & 35 & 30\end{array}$ 


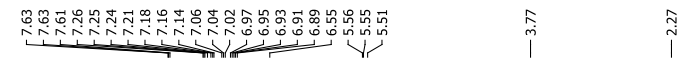

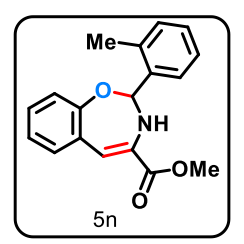

$400 \mathrm{MHz}, \mathrm{CDCl}_{3}$

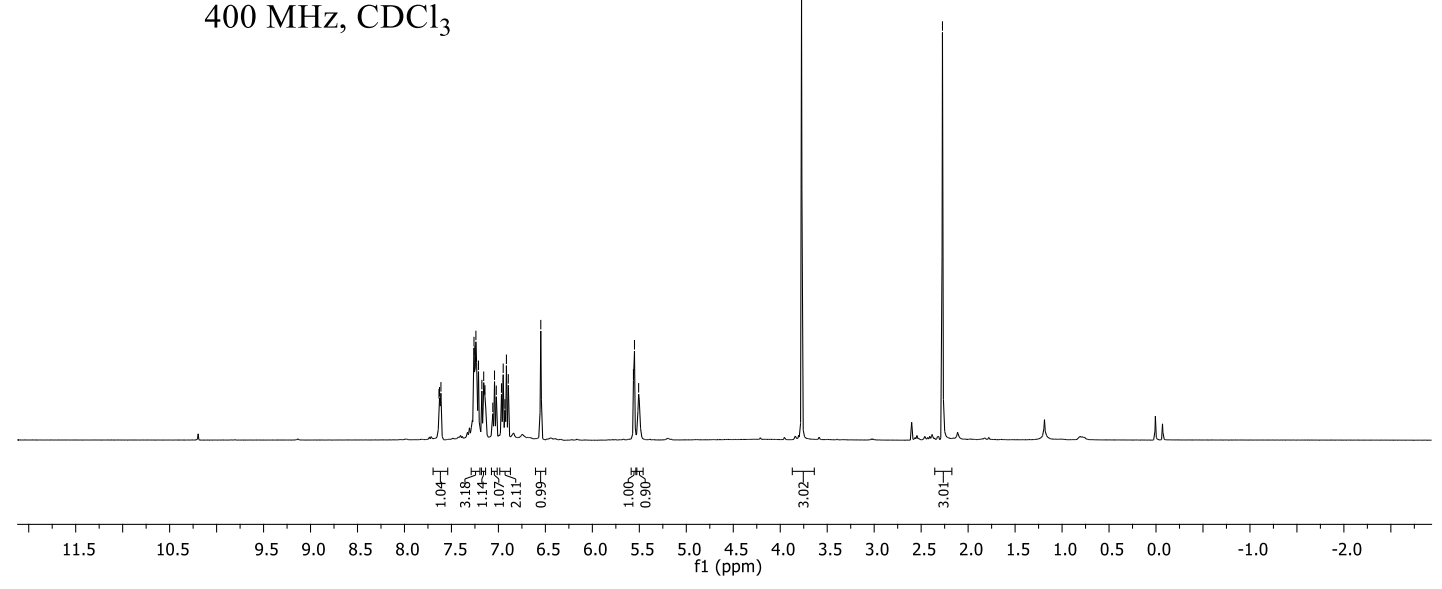

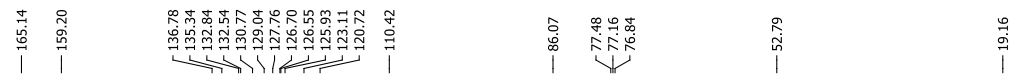

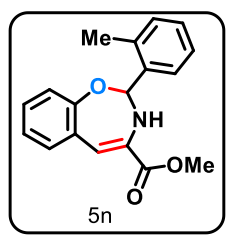

$101 \mathrm{MHz}, \mathrm{CDCl}_{3}$

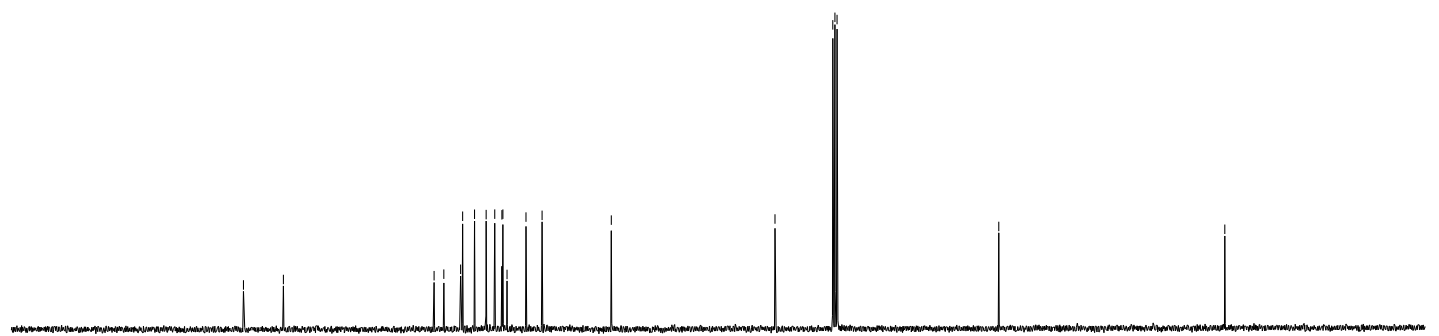

$\begin{array}{llllllllllllllllllll}190 & 180 & 170 & 160 & 150 & 140 & 130 & 120 & 110 & \underset{\mathrm{f} 1(\mathrm{ppm})}{100} & 80 & 70 & 60 & 50 & 40 & 30 & 20 & 10 & 0 & -10\end{array}$ 


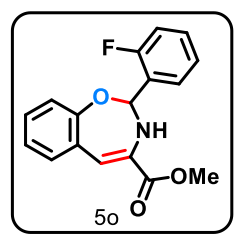

$400 \mathrm{MHz}, \mathrm{CDCl}_{3}$
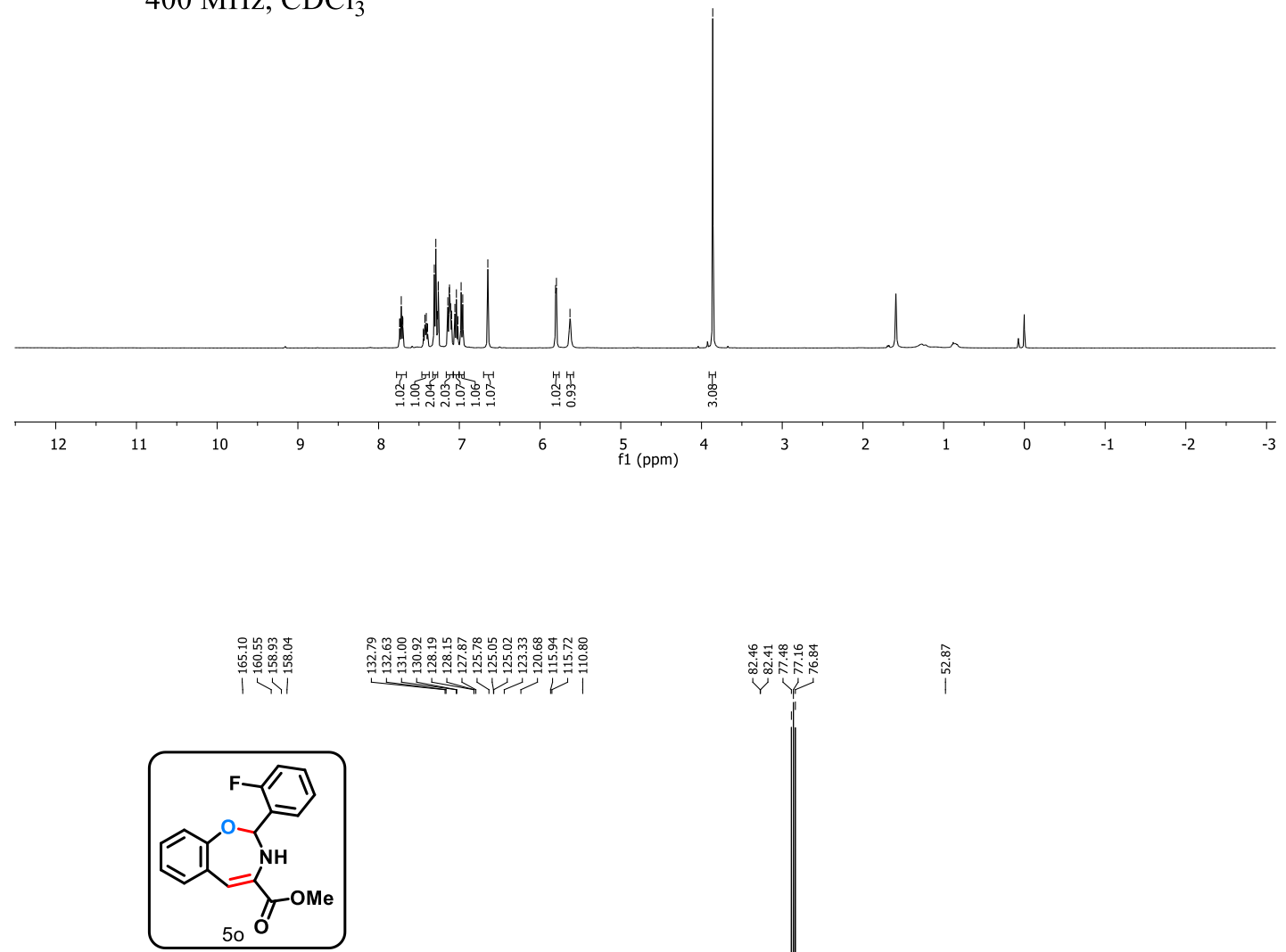

$101 \mathrm{MHz}, \mathrm{CDCl}_{3}$
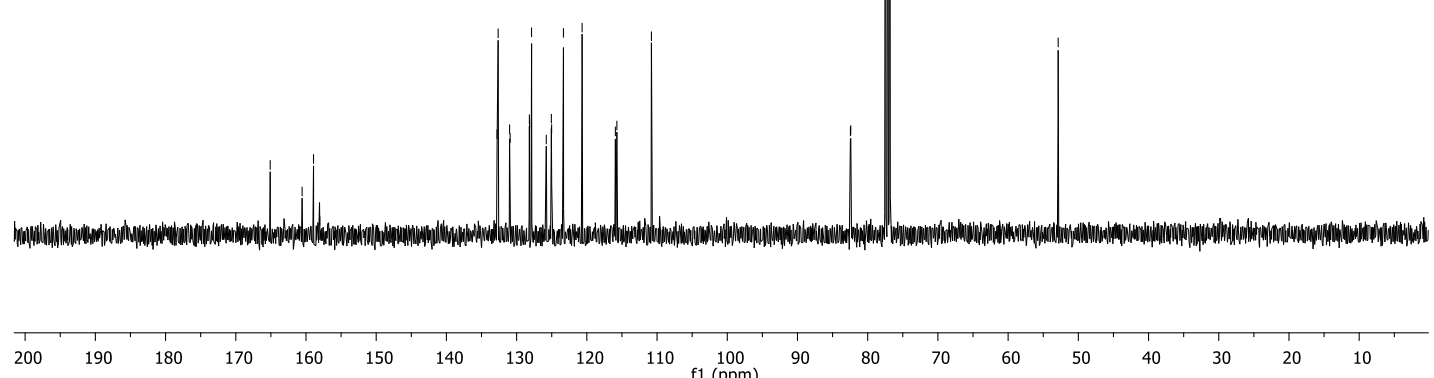


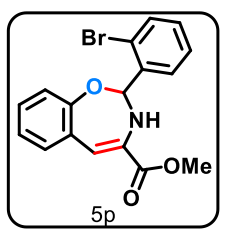

$400 \mathrm{MHz}, \mathrm{CDCl}_{3}$
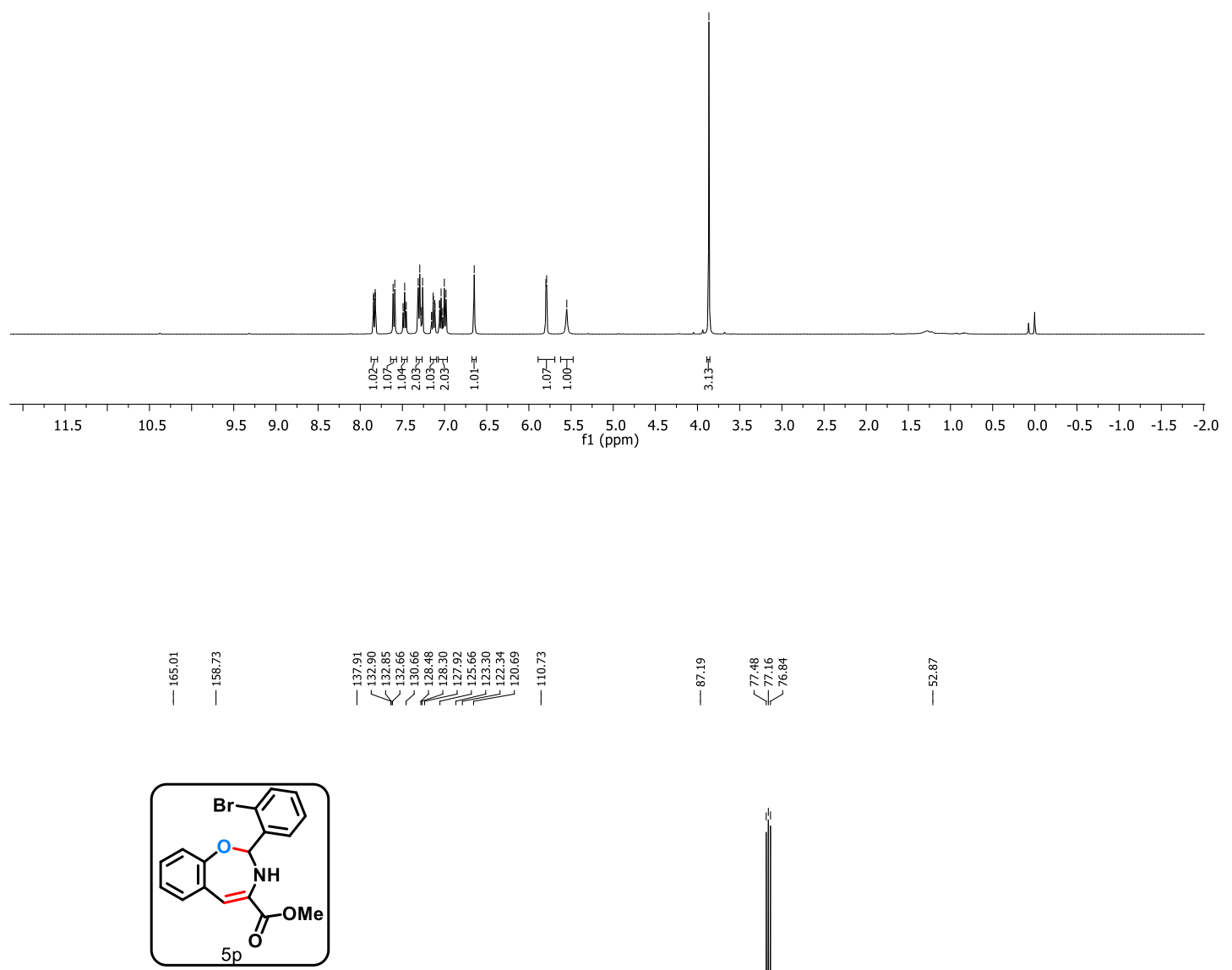

$101 \mathrm{MHz}, \mathrm{CDCl}_{3}$

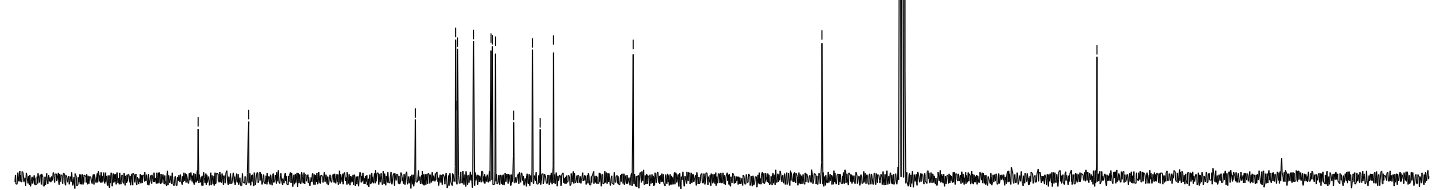




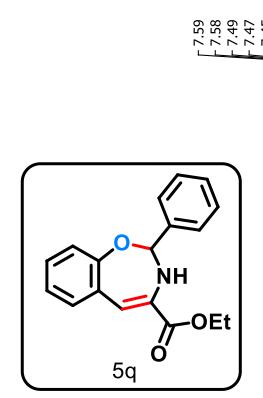

$400 \mathrm{MHz}, \mathrm{CDCl}_{3}$
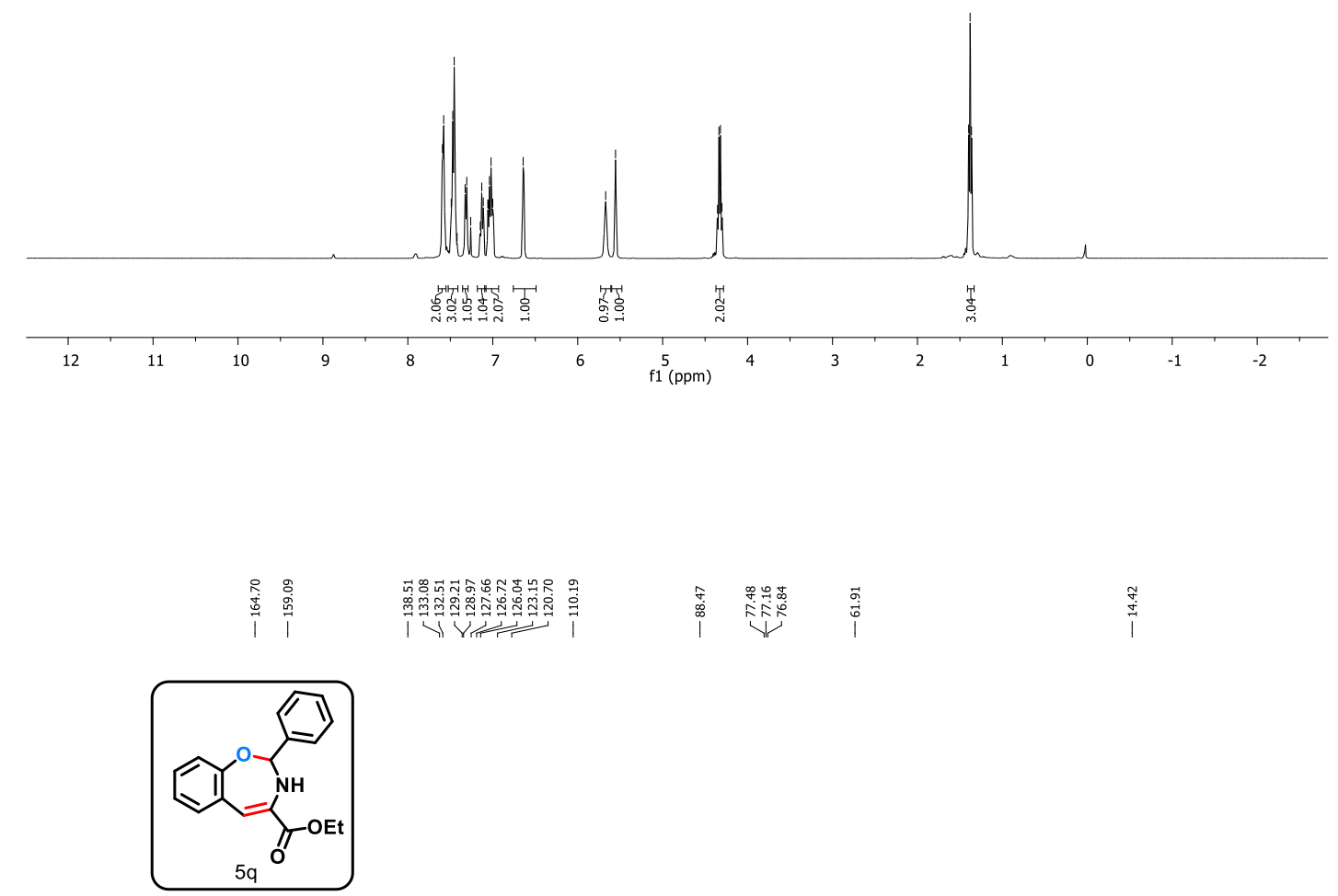

$101 \mathrm{MHz}, \mathrm{CDCl}_{3}$
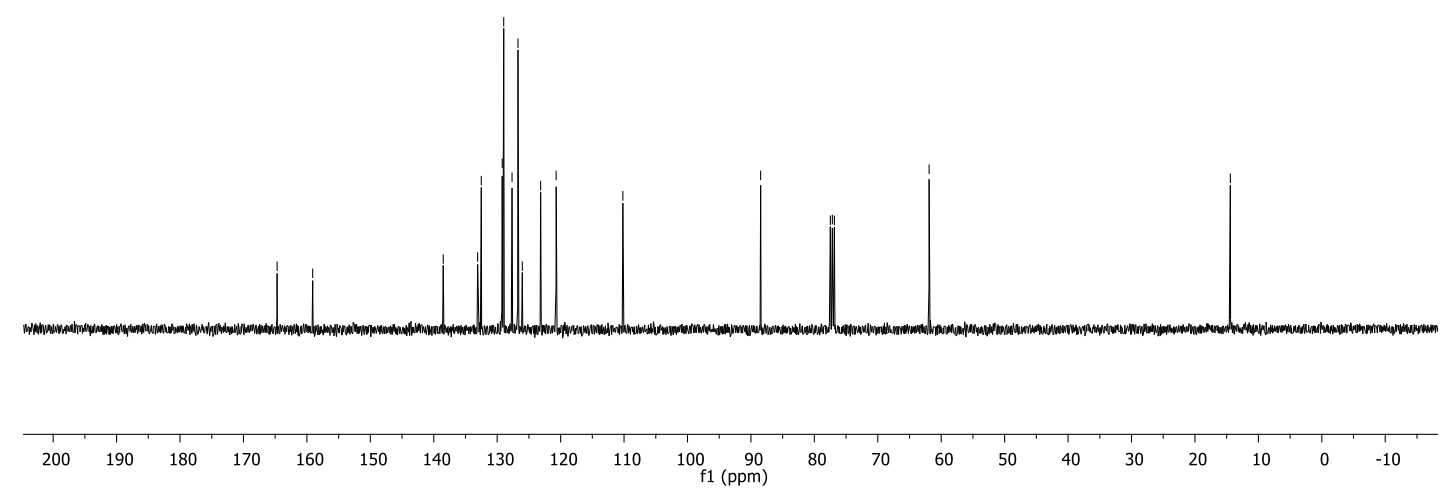


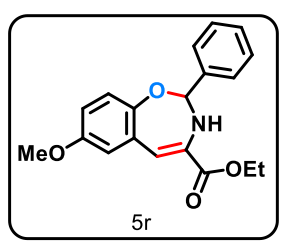

$400 \mathrm{MHz}, \mathrm{CDCl}_{3}$

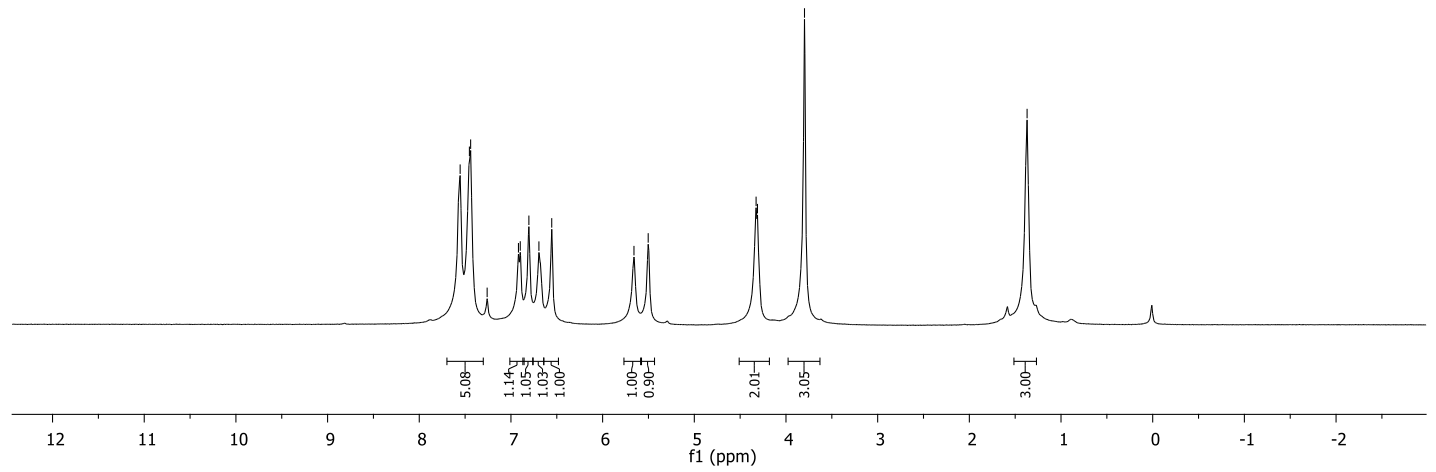

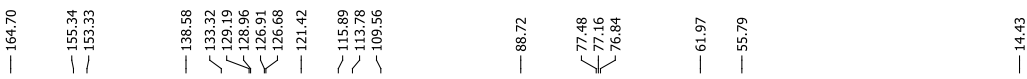

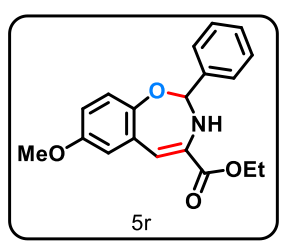

$101 \mathrm{MHz}, \mathrm{CDCl}_{3}$
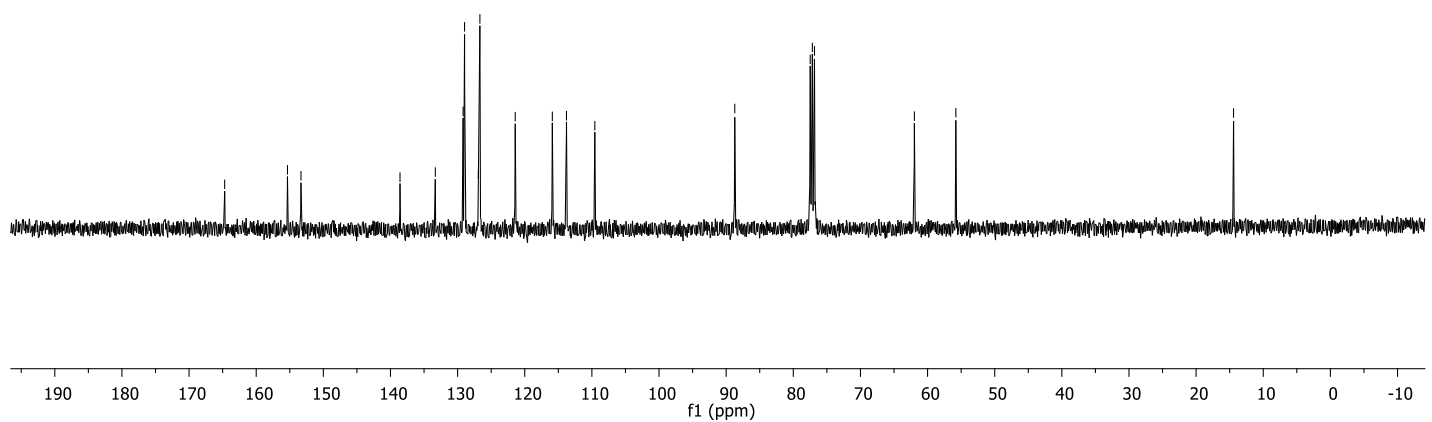


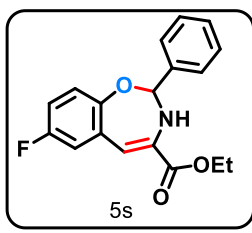

$400 \mathrm{MHz}, \mathrm{CDCl}_{3}$

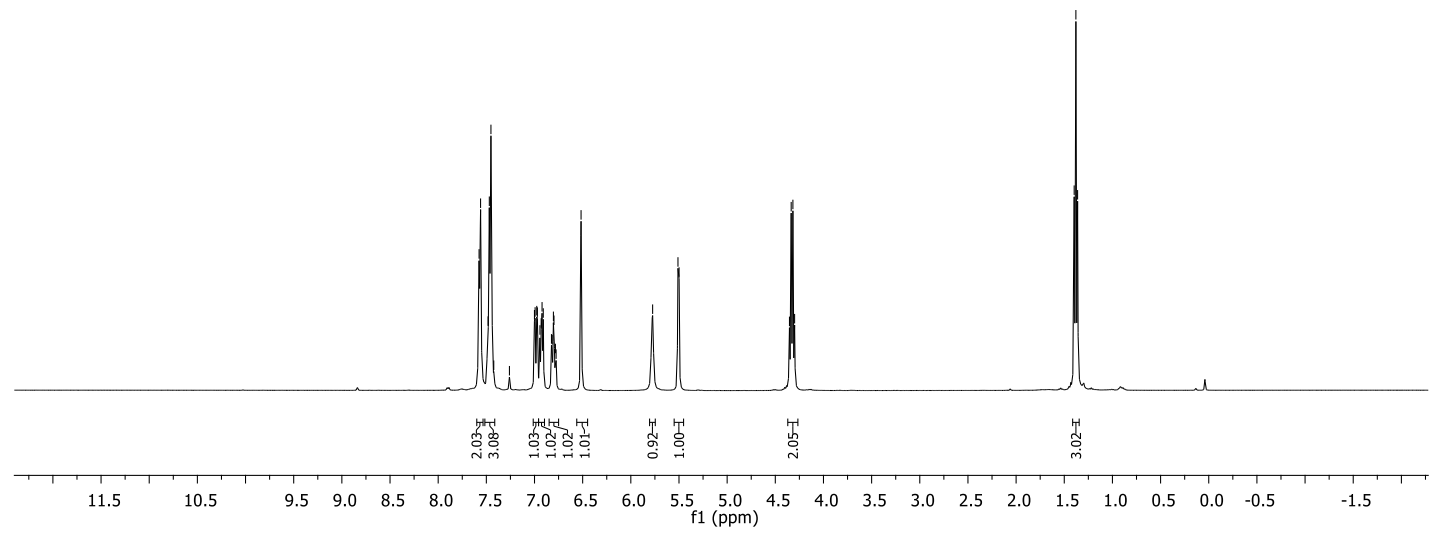

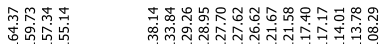

|

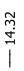

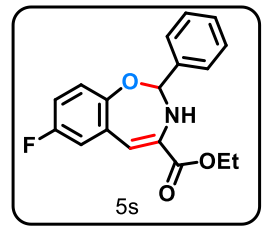

$101 \mathrm{MHz}, \mathrm{CDCl}_{3}$

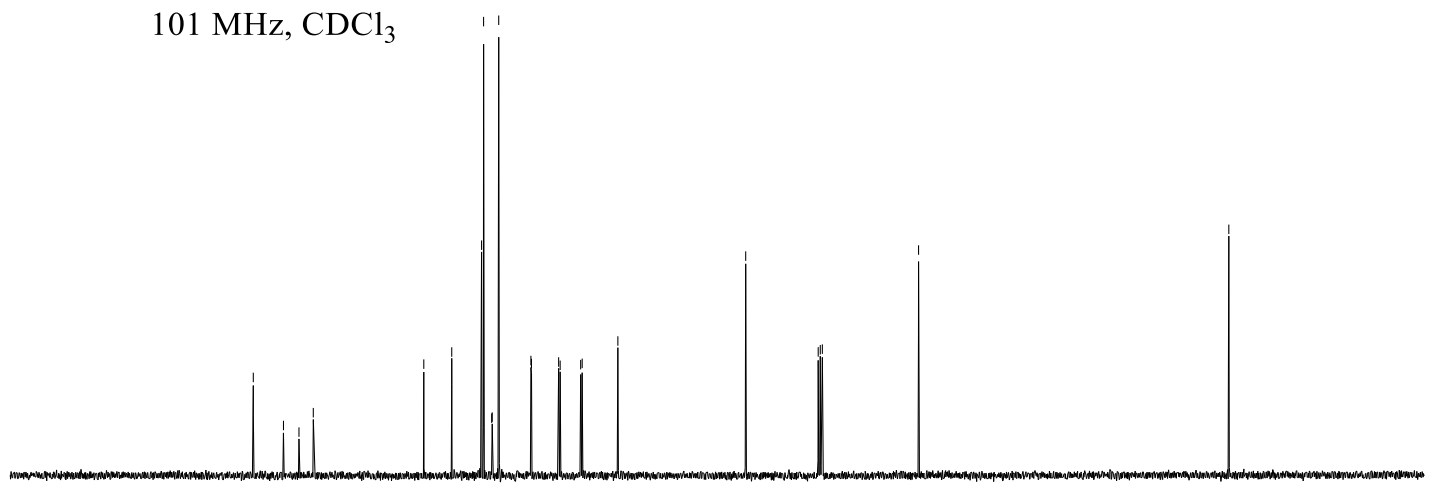

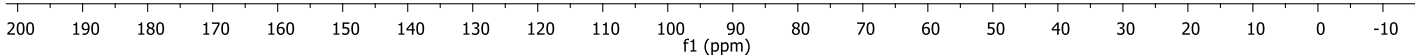




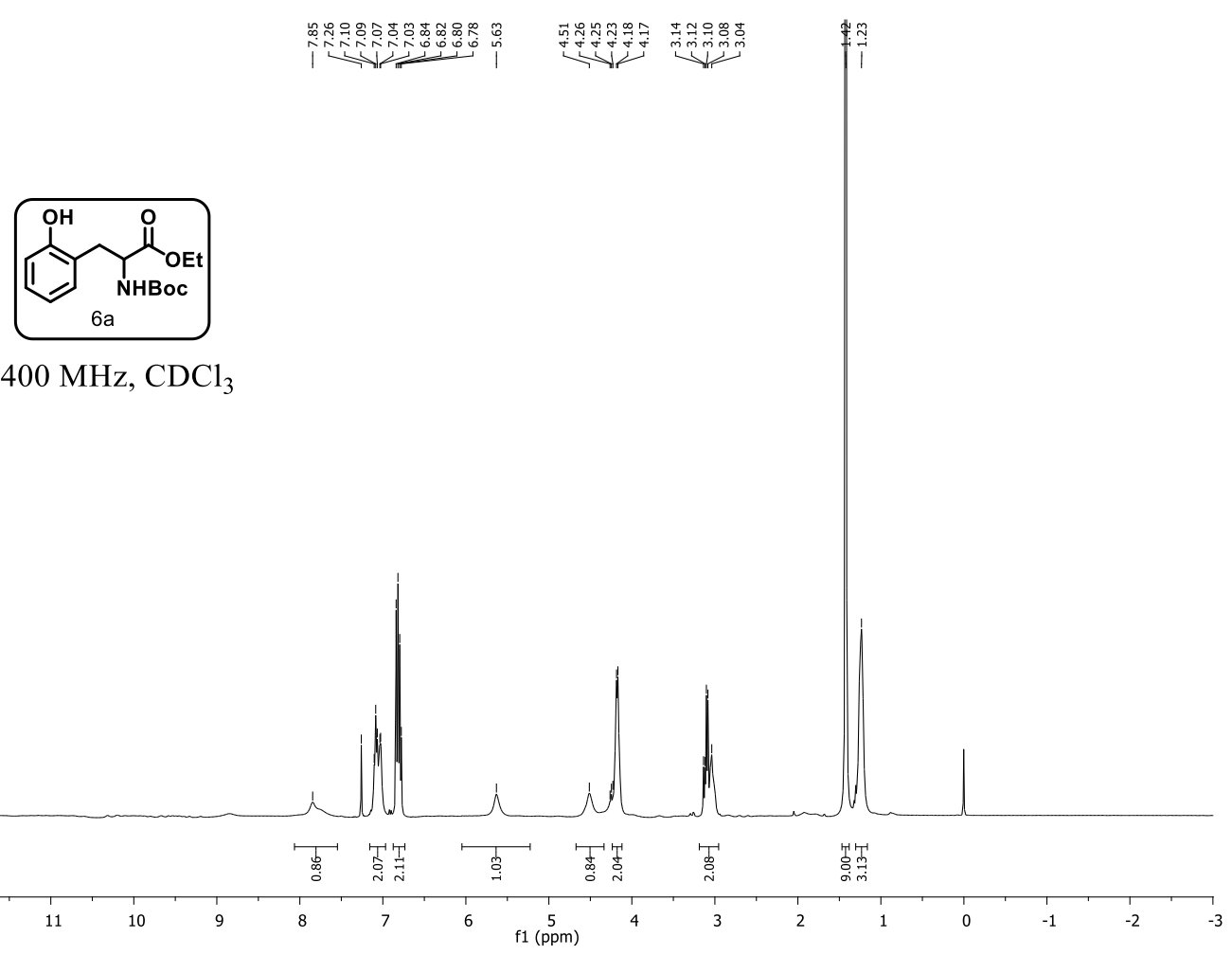

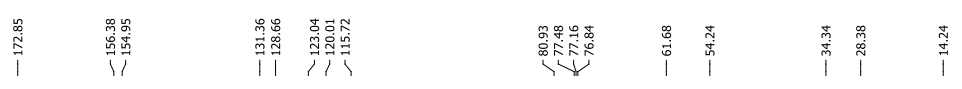

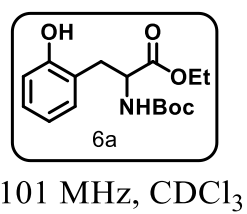

$101 \mathrm{MHz}, \mathrm{CDCl}_{3}$

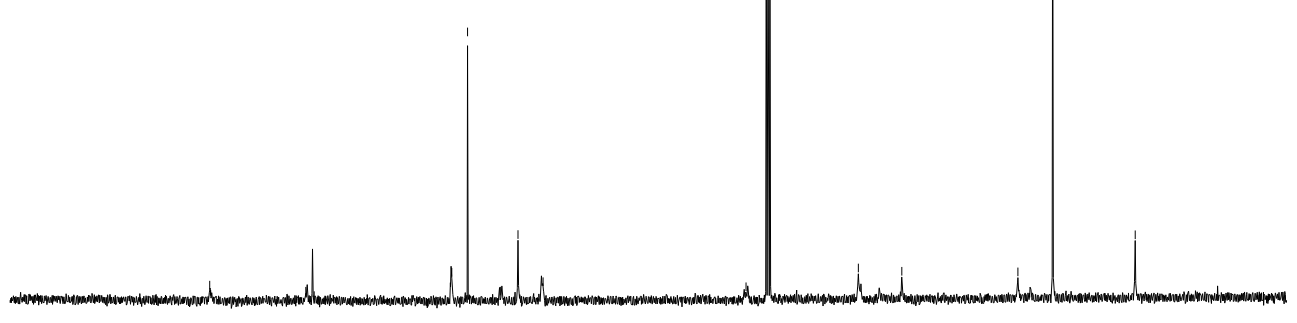

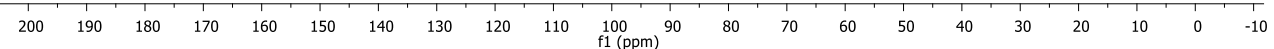




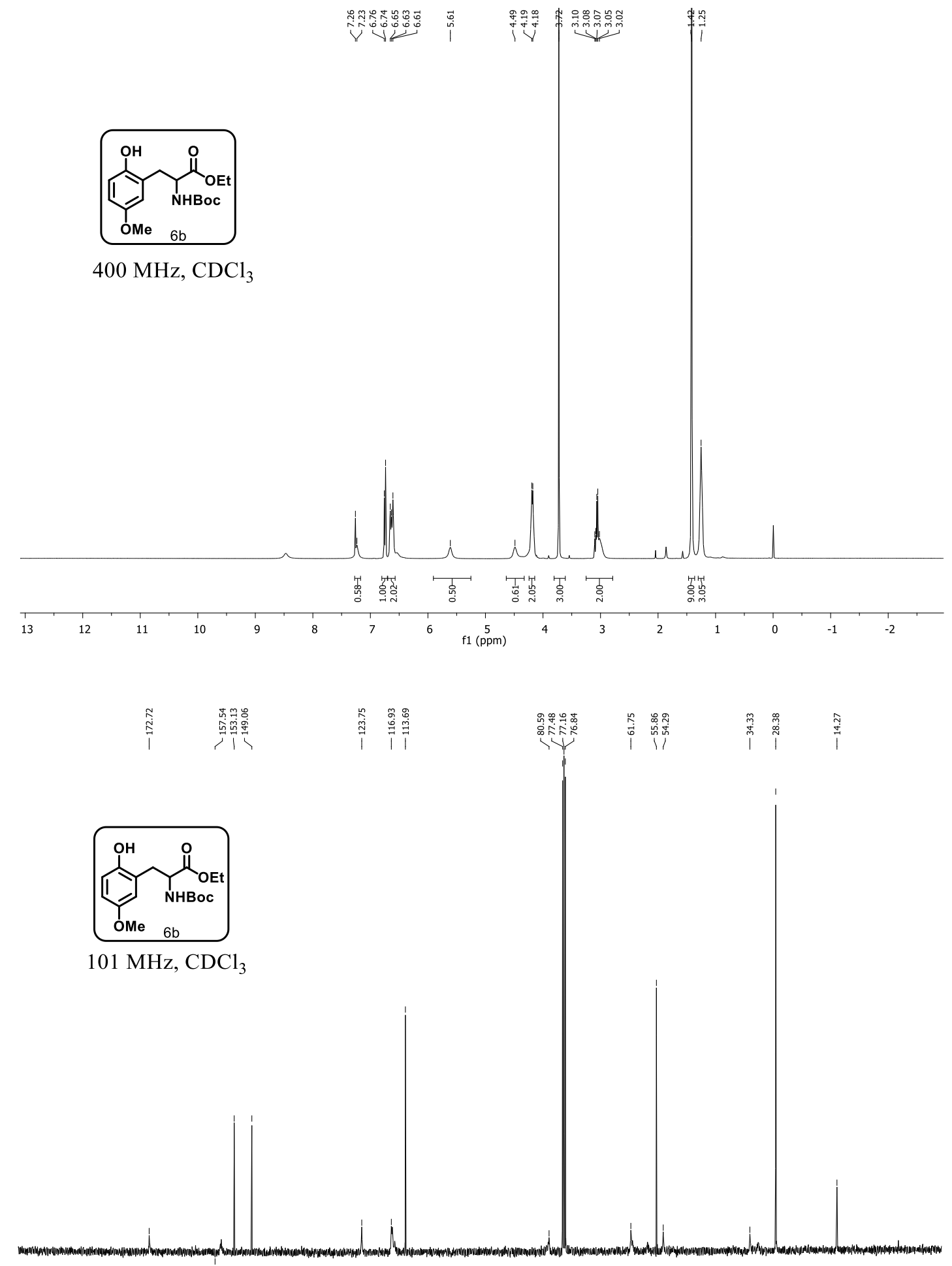

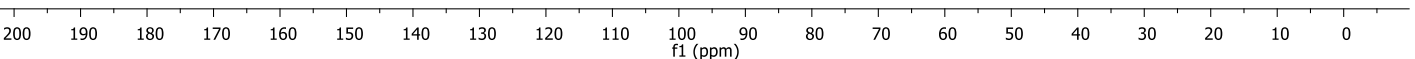



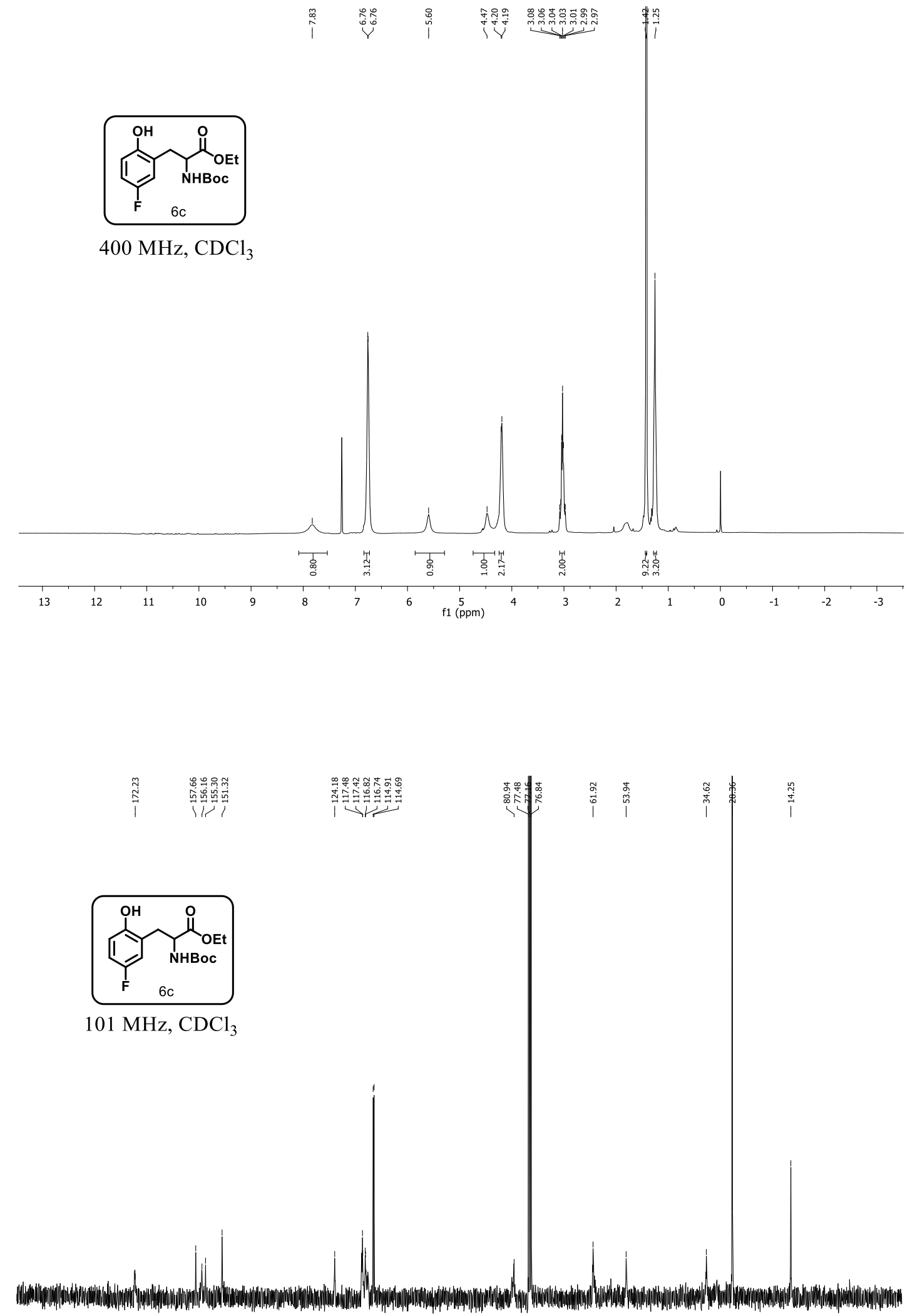

$\begin{array}{lllllllllll}200 & 190 & 180 & 170 & 160 & 150 & 140 & 130 & 120 & 110 & 100 \\ \mathrm{f} 1(\mathrm{ppm}) & 90\end{array}$ 


\section{$F^{19}$ Spectra of compounds}

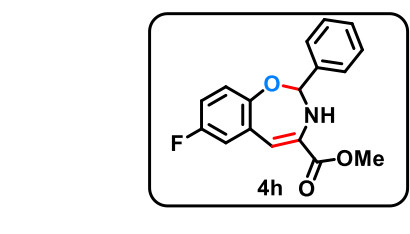

$470 \mathrm{MHz}, \mathrm{CDCl}_{3}$
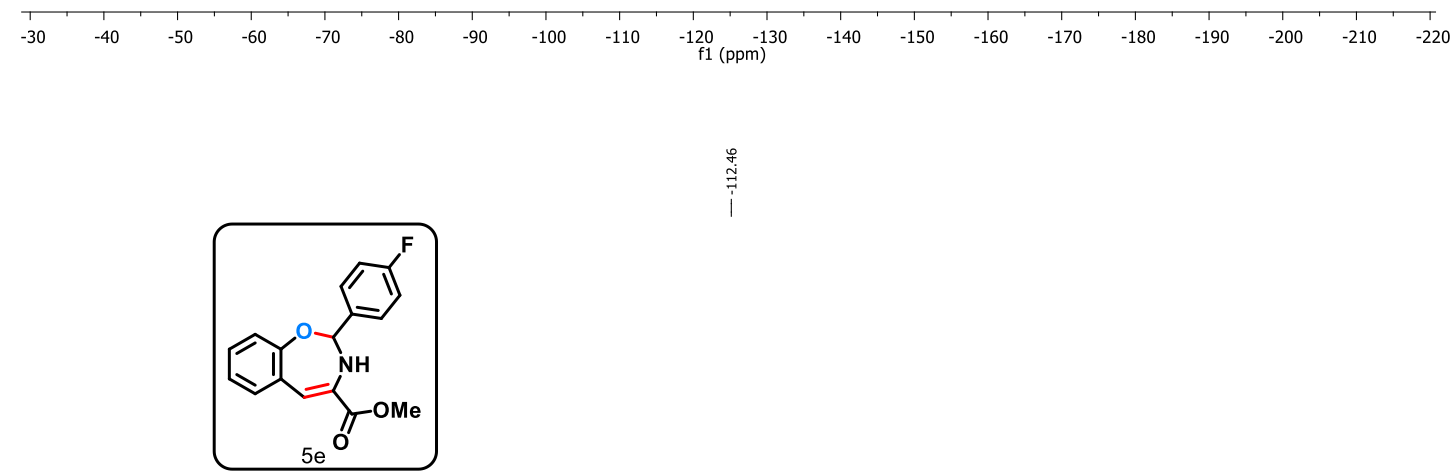

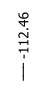

$470 \mathrm{MHz}, \mathrm{CDCl}_{3}$

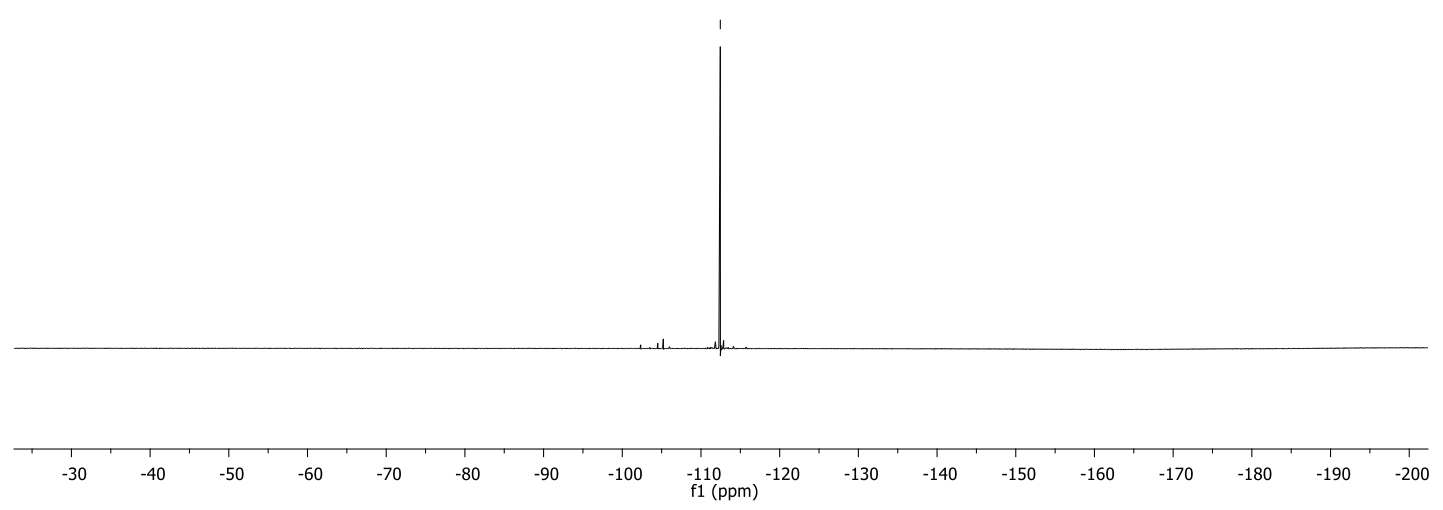




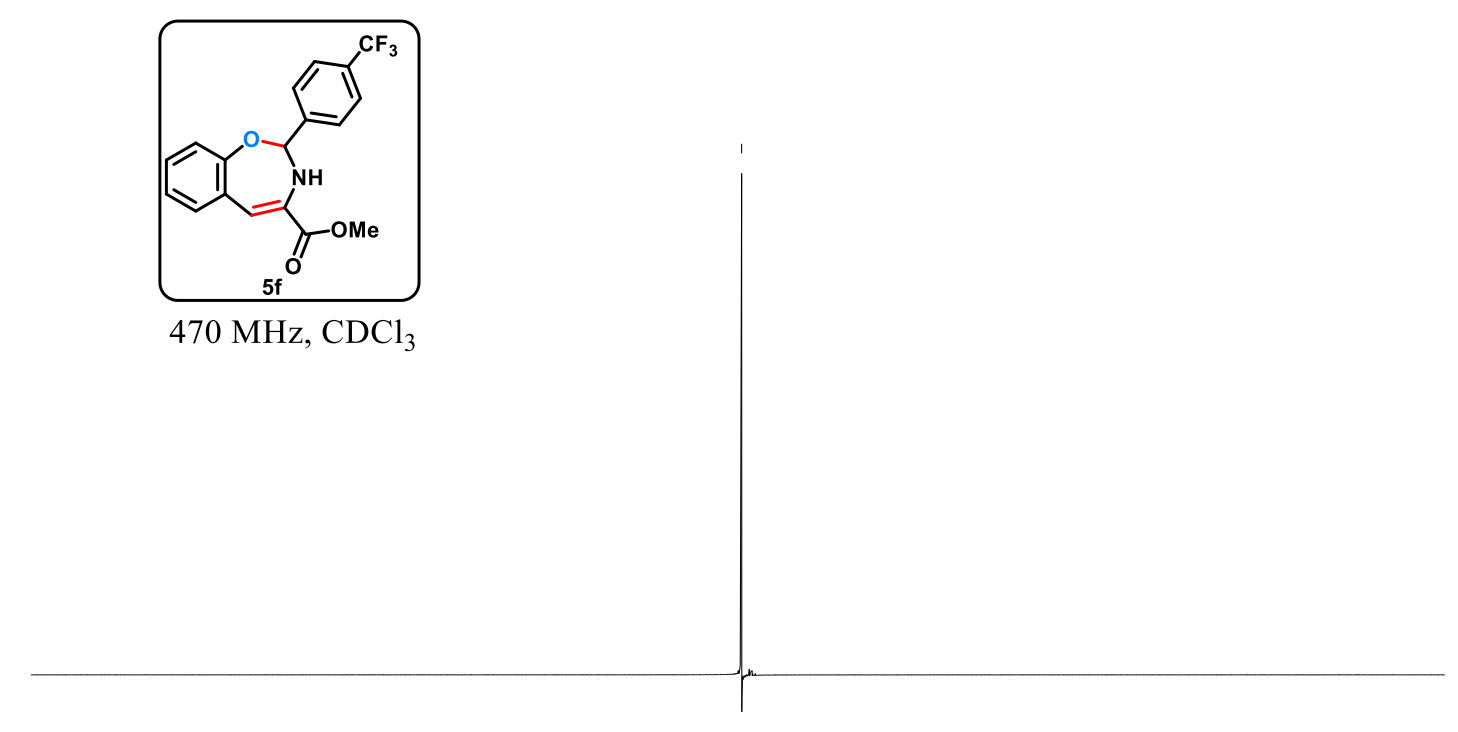

$470 \mathrm{MHz}, \mathrm{CDCl}_{3}$

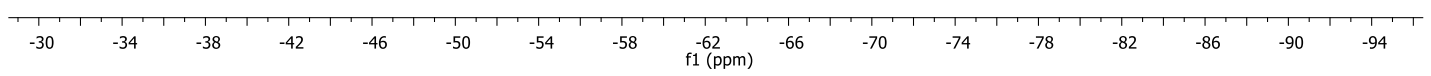

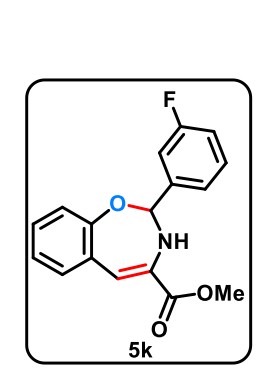

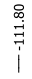

$470 \mathrm{MHz}, \mathrm{CDCl}_{3}$

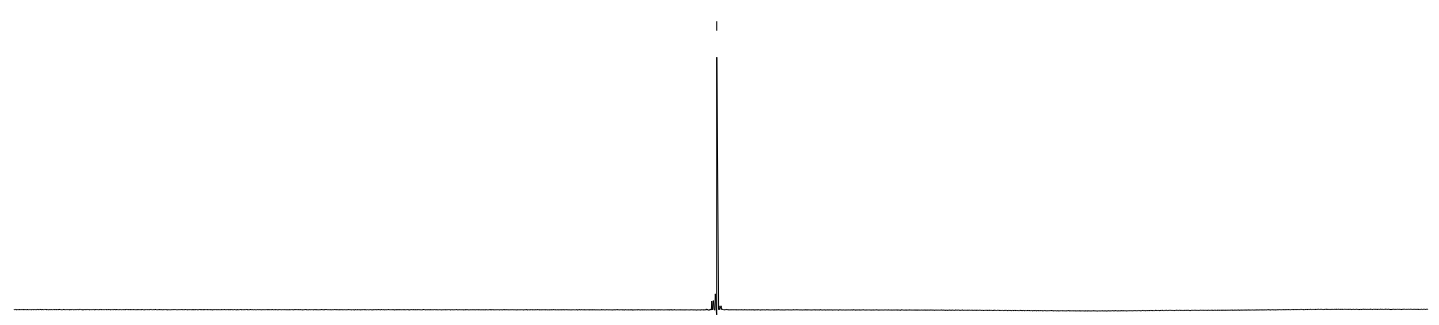

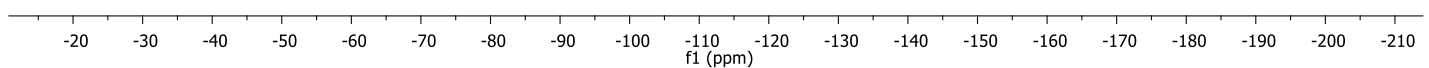




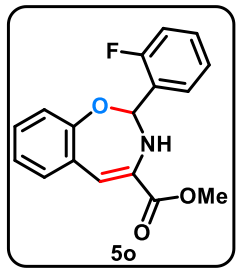

$470 \mathrm{MHz}, \mathrm{CDCl}_{3}$
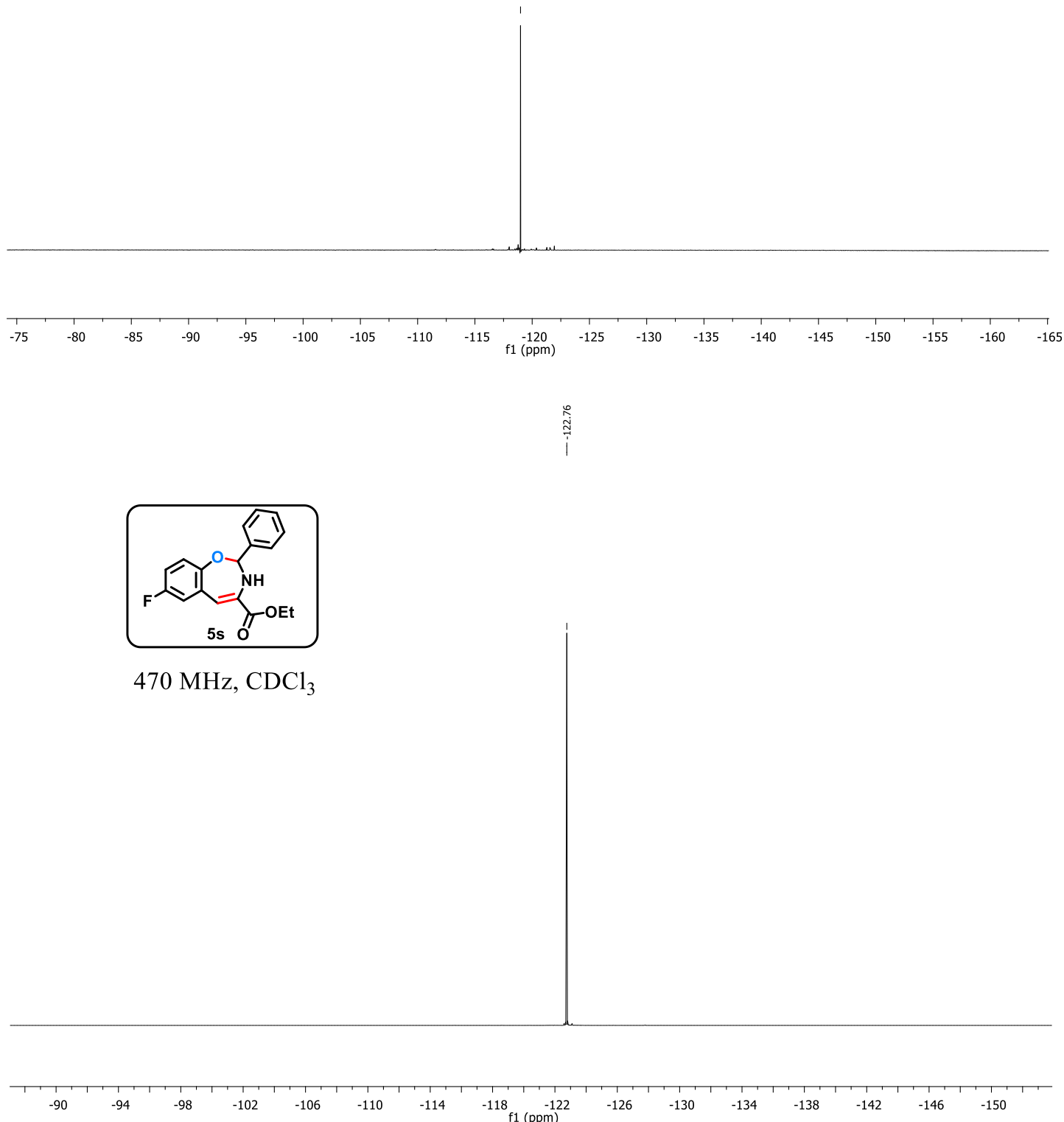